\title{
Regio- and Diastereoselective Copper-Catalyzed Carbometallation of Cyclopropenylsilanes
}

\author{
Yair Cohen and Ilan Marek* \\ Schulich Faculty of Chemistry, Technion - Israel Institute of Technology, Technion City, Haifa, \\ 3200009, Israel
}

Supporting Information 
Table of contents

1. General experimental details.

2. General procedure for the copper-catalyzed carbomagnesiation of cyclopropenylsilanes 11a-11h.

3. Characterization of cyclopropylsilanes 12a-12z.

4. General procedure for the reduction of cyclopropenylsilanes and characterization of cyclopropenylsilanes 11a-11h.

5. General procedure for the silylation of cyclopropenes characterization of cyclopropenylsilanes 13a-13h.

6. Crystal data and structure refinement for compund 12c (CCDC 1942906)

7. References.

8. NMR spectra of cyclopropylsilanes 12a-12z.

9. NMR spectra of cyclopropenylsilanes 11a-11h.

10. NMR spectra of cyclopropenylsilanes 13a-13h. 


\section{General experimental details}

Unless stated otherwise, reactions were conducted in flame-dried glassware under a positive pressure of argon. $\mathrm{Et}_{2} \mathrm{O}$ and THF were dried from Pure-Solv ${ }^{\circledR}$ Purification System (Innovative Technology@). All other reagents were purchased from Aldrich, Strem, Acros, and Alfa Aesar, and used as received. All Grignard reagents (beside $\mathrm{MeMgBr}$ ) were prepared from corresponding commercially available alkyl bromides, and freshly titrated before use. Compound $\mathbf{1 1 b}$ was synthesized according to a known procedure ${ }^{1}$. Thin-layer chromatography (TLC) was conducted with E. Merck silica gel 60 F254 pre-coated plates, $(0.25 \mathrm{~mm})$ and visualized by exposure to UV light $(254 \mathrm{~nm})$ or stained with anisaldehyde, phosphomolybdic acid, or potassium permanganate. Column chromatography was performed using Fluka silica gel $60 \AA$ ( $40-63 \mu \mathrm{m}$, 230-400 mesh). All NMR spectra were recorded on Bruker spectrometers (AVIII 400) and are reported relative to deuterated solvent signals. Chemical shifts are reported in parts per million (ppm) with respect to the residual solvent signal $\mathrm{CDCl}_{3}\left({ }^{1} \mathrm{H}\right.$ NMR: $\left.\delta=7.26 ;{ }^{13} \mathrm{C} \mathrm{NMR:} \delta=77.16\right) .{ }^{29} \mathrm{Si}$ Chemical shifts are reported in parts per million (ppm) with respect to tetramethylsilane $\left({ }^{29} \mathrm{Si} N \mathrm{NMR}: \delta=0\right)$. Peak multiplicities are reported as follows: $s=$ singlet, $b s=$ broad singlet, $d=$ doublet, $t=$ triplet, $q=$ quartet, quin = quintet, sex = sextet, $\operatorname{sep}=$ septet, $\mathrm{dd}=$ doublet of doublets, $\mathrm{td}=$ triplet of doublets, $\mathrm{ddd}=$ doublet of doublets of doublets, $m=$ multiplet. High-resolution mass spectra (HRMS) were obtained by the mass spectrometry facility at the Technion. Reactions were monitored by gas chromatography spectrometry (GC) using an Agilent Technologies 7820A GC with an Agilent Technologies 19091J-413 (30 m × $0.3 \mathrm{~mm}$ ) column. HPLC chromatograms were recorded using Agilent@ 1100 Series line. 
2. General procedure for the copper-catalyzed carbomagnesiation of cyclopropenylsilanes

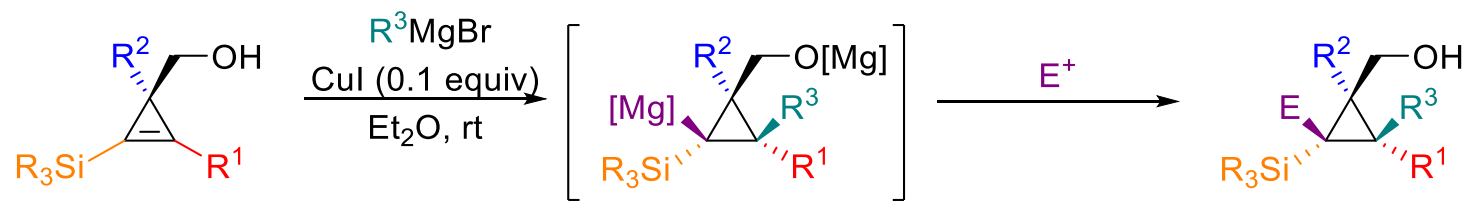

Copper iodide ( $9.5 \mathrm{mg}, 0.05 \mathrm{mmol}, 0.1$ eq.) was placed in a flame-dried Schlenk tube. The tube was purged with argon three times. $\mathrm{Et}_{2} \mathrm{O}(5 \mathrm{~mL}, 0.1 \mathrm{M})$ was added through a septum and the mixture was stirred at room temperature, followed by addition of cyclopropenylsilane $(0.5 \mathrm{mmol}, 1 \mathrm{eq}$.). Then alkyl magnesium bromide solution in $\mathrm{Et}_{2} \mathrm{O}$ ( 3 eq.) was added dropwise. During addition the colorless suspension became homogenous yellowish solution and upon stirring darkened to brown or black solution. Upon full consumption of the cyclopropenylsilane (followed by GC, ca. 2 h) the reaction was quenched with a saturated aqueous $\mathrm{NH}_{4} \mathrm{Cl}$ solution.

In case of electrophile incorporation:

Allyl bromide: Upon full consumption of the cyclopropenylsilane (followed by GC, ca. $2 \mathrm{~h}$ ) allyl bromide ( $210 \mu \mathrm{L}, 2.5 \mathrm{mmol}, 5 \mathrm{eq})$ was added dropwise to the reaction mixture. Upon full consumption of the hydrolysis product (followed by GC, ca. $1 \mathrm{~h}$ ) the reaction was acidified with an saturated aqueous ammonium chloride solution.

Carbon dioxide: Upon full consumption of the cyclopropenylsilane (followed by GC, ca. 2 h) carbon dioxide was bubbled through the solution, and a balloon of carbon dioxide was placed instead of the argon inlet. Upon full consumption of the hydrolysis product (followed by TLC, 40\% EtOAc/ petroleum ether, $p$-anisaldehyde, ca. $1 \mathrm{~h}$ ) the reaction was acidified with $1 \mathrm{M} \mathrm{HCl}$ solution.

lodine: Upon full consumption of the cyclopropenylsilane (followed by GC, ca. $2 \mathrm{~h}$ ) iodine ( 380 $\mathrm{mg}, 1.5 \mathrm{mmol}, 3 \mathrm{eq})$ was added dropwise as solution in THF $(2 \mathrm{~mL})$ to reaction mixture. Upon full consumption of the hydrolysis product (followed by GC, ca. $1 \mathrm{~h}$ ) the reaction was quenched with saturated aqueous $\mathrm{Na}_{2} \mathrm{~S}_{2} \mathrm{O}_{3}$ solution.

The aqueous layer was extracted three times with $\mathrm{Et}_{2} \mathrm{O}$ and the combined organic phases were washed with brine, dried over $\mathrm{Na}_{2} \mathrm{SO}_{4}$, filtered, and concentrated under reduced pressure. Crude mixture was then purified by flash chromatography using EtOAc / petroleum ether as eluent. 
3. Characterization of cyclopropylsilanes $\mathbf{1 2 a - 1 2 z}$

(2-butyl-3-(dimethyl(phenyl)silyl)-2-methylcyclopropyl)methanol (12a)

Synthetic procedure for the copper-catalyzed carbomagnesiation of cyclopropenylsilanes on preparative scale:

Copper iodide (90 mg, $0.5 \mathrm{mmol}, 0.1$ eq.) was placed in a flame-dried $250 \mathrm{~mL} 3$ neck flask. The flask was purged with argon three times. $\mathrm{Et}_{2} \mathrm{O}(50 \mathrm{~mL}, 0.1 \mathrm{M})$ was added through a septum and the mixture was stirred at room temperature, followed by addition of cyclopropenylsilane ( 5 mmol, 1eq.). Then, a solution of alkyl magnesium bromide ( 3 eq.) in $\mathrm{Et}_{2} \mathrm{O}$ was added dropwise. During addition, the colorless suspension became homogenous yellowish and upon stirring darkened to brown or black solution. Upon full consumption of the cyclopropenylsilane (followed by $\mathrm{GC}$, ca. $2 \mathrm{~h}$ ) the reaction was quenched with a saturated aqueous $\mathrm{NH}_{4} \mathrm{Cl}$ solution. The aqueous layer was extracted three times with $\mathrm{Et}_{2} \mathrm{O}$ and the combined organic phases were washed with brine, dried over $\mathrm{Na}_{2} \mathrm{SO}_{4}$, filtered, and concentrated under reduced pressure. Crude mixture was then purified by flash chromatography using EtOAc / petroleum ether as eluent.

Scale, physical description, yield: $5 \mathrm{mmol}$, colorless oil, $79 \%$ yield, $1.1 \mathrm{gr}$. Flash chromatography: 5 to 10 \% EtOAc / petroleum ether. ${ }^{1} \mathrm{H}$ NMR $\left(400 \mathrm{MHz}, \mathrm{CDCl}_{3}\right) \mathbf{8}:-0.43(1 \mathrm{H}, \mathrm{d}, \mathrm{J}=7.24 \mathrm{~Hz}), 0.28(3 \mathrm{H}, \mathrm{s}), 0.31(3 \mathrm{H}$, s), $0.83(3 \mathrm{H}, \mathrm{t}, \mathrm{J}=7.14 \mathrm{~Hz}), 0.99-1.03(1 \mathrm{H}, \mathrm{m}), 1.15(3 \mathrm{H}, \mathrm{s}), 1.20-1.38(6 \mathrm{H}, \mathrm{m}), 3.55(1 \mathrm{H}, \mathrm{dd}, \mathrm{J}=8.36,11.33$ $\mathrm{Hz}), 3.73(1 \mathrm{H}, \mathrm{dd}, \mathrm{J}=6.38,11.39 \mathrm{~Hz}), 7.34-7.36(3 \mathrm{H}, \mathrm{m}), 7.52-7.54(2 \mathrm{H}, \mathrm{m}) .{ }^{13} \mathrm{C} \mathbf{N M R}\left(101 \mathrm{MHz}, \mathrm{CDCl}_{3}\right)$ 8: 1.47, -1.07, 14.22, 18.23, 19.37, 23.00, 26.86, 29.89, 31.46, 38.66, 64.91, 127.90, 128.94, 133.76, 140.19.

${ }^{29}$ Si NMR (80 MHz, $\mathrm{CDCl}_{3}$ ) 8: -4.58. HRMS (APCl): m/z calculated for $\mathrm{C}_{17} \mathrm{H}_{27} \mathrm{Si}[\mathrm{M}-\mathrm{OH}]^{+}: 259.1877$, found 259.1911.

(allyl-3-butyl-2-(dimethyl(phenyl)silyl)-3-methylcyclopropyl)methanol (12b)

Scale, physical description, yield: $0.5 \mathrm{mmol}$, colorless oil, $78 \%$ yield, $124 \mathrm{mg}$. Flash chromatography: 5 to $10 \%$ EtOAc / petroleum ether. ${ }^{1} \mathrm{H}$ NMR $\left(400 \mathrm{MHz}, \mathrm{CDCl}_{3}\right) \delta: 0.35(3 \mathrm{H}, \mathrm{s}), 0.39(3 \mathrm{H}, \mathrm{s}), 0.82(3 \mathrm{H}, \mathrm{t}, \mathrm{J}=\mathrm{Hz})$, $1.03(1 \mathrm{H}, \mathrm{t}, \mathrm{J}=\mathrm{Hz}), 1.11(3 \mathrm{H}, \mathrm{s}), 1.17-1.22(4 \mathrm{H}, \mathrm{m}), 1.36-1.41(1 \mathrm{H}, \mathrm{m}), 1.43-1.51(1 \mathrm{H}, \mathrm{m}), 2.07-2.13(1 \mathrm{H}, \mathrm{m})$, 2.22-2.28 (1H, m), 3.68-6.78 (2H, m), 4.90-4.99 (2H, m), 5.67-5.77 (1H, m), 7.32-7.34 (3H, m), 7.51-7.53 (2H, m). ${ }^{13} \mathrm{C}$ NMR (101 MHz, $\left.\mathrm{CDCl}_{3}\right) \delta:-0.56,0.23,14.25,14.27,19.62,22.98,28.79,29.76,32.16,33.06$, $40.20,60.38,115.91,127.74,128.72,134.49,138.71,140.29 .{ }^{29}$ Si NMR (80 MHz, $\mathrm{CDCl}_{3}$ ) 8: 1.18. HRMS (APCI): $\mathrm{m} / \mathrm{z}$ calculated for $\mathrm{C}_{20} \mathrm{H}_{33} \mathrm{SOi}[\mathrm{M}+\mathrm{H}]^{+}$: 317.2295, found 317.2298. 
2-butyl-1-(dimethyl(phenyl)silyl)-3-(hydroxymethyl)-2-methylcyclopropanecarboxylic acid (12c)

Scale, physical description, yield: $0.5 \mathrm{mmol}$, white solid. $40 \%$ yield, $65 \mathrm{mg}$. Flash chromatography: $40 \%$ EtOAc / petroleum ether. ${ }^{1} \mathbf{H}$ NMR (400 MHz, $\left.\mathbf{C D C l}_{3}\right)$ 8: $0.45(3 \mathrm{H}, \mathrm{s}), 0.46(3 \mathrm{H}, \mathrm{s}), 0.78(3 \mathrm{H}, \mathrm{t}, \mathrm{J}=7.27 \mathrm{~Hz})$, $1.10(3 \mathrm{H}, \mathrm{s}), 1.12-1.15(2 \mathrm{H}, \mathrm{m}), 1.35-1.39(2 \mathrm{H}, \mathrm{m}), 1.47-1.54(2 \mathrm{H}, \mathrm{m}), 3.63(1 \mathrm{H}, \mathrm{dd}, \mathrm{J}=10.48,12.10 \mathrm{~Hz}), 3.92$ $(1 \mathrm{H}, \mathrm{dd}, \mathrm{J}=6.11,12.11 \mathrm{~Hz}), 7.34-7.35(3 \mathrm{H}, \mathrm{m}), 7.56-7.58(2 \mathrm{H}, \mathrm{m}) .{ }^{13} \mathrm{C} \mathrm{NMR}\left(101 \mathrm{MHz}, \mathrm{CDCl}_{3}\right)$ 8: -1.92, 14.07 , 16.65 , 22.65 , 29.23 , 31.77 , 31.97 , 35.03 , 38.48 , 60.80 , 127.94 , 129.51 , 134.62 , 136.89.

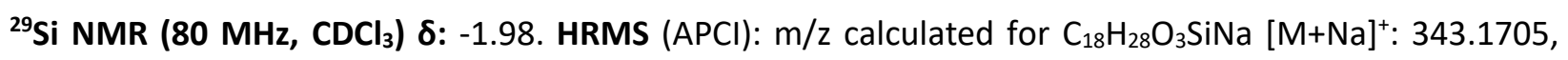
found 343.1716 .

(2-butyl-3-(dimethyl(phenyl)silyl)-2-ethylcyclopropyl)methanol (12d)

Scale, physical description, yield: $0.5 \mathrm{mmol}$, colorless oil. $89 \%$ yield, 139mg. (contains minor leftovers of starting material, 6\%) Flash chromatography: 5 to $10 \%$ EtOAc / petroleum ether. ${ }^{1} \mathbf{H} \mathbf{~ N M R ~ ( 4 0 0 ~} \mathbf{M H z}$, $\left.\mathrm{CDCl}_{3}\right)$ ס: - $0.47(1 \mathrm{H}, \mathrm{d}, \mathrm{J}=7.32 \mathrm{~Hz}), 0.29(3 \mathrm{H}, \mathrm{s}), 0.32(3 \mathrm{H}, \mathrm{s}), 0.83(3 \mathrm{H}, \mathrm{t}, \mathrm{J}=14.09 \mathrm{~Hz}), 0.92(3 \mathrm{H}, \mathrm{t}, \mathrm{J}=7.30 \mathrm{~Hz})$, 1.02-1.12 $(3 \mathrm{H}, \mathrm{m}), 1.21-1.25(3 \mathrm{H}, \mathrm{m}), 1.31-1.34(1 \mathrm{H}, \mathrm{m}), 1.52-1.54(1 \mathrm{H}, \mathrm{m}), 1.60-1.68(1 \mathrm{H}, \mathrm{m}), 3.58(1 \mathrm{H}, \mathrm{dd}$, $\mathrm{J}=8.26,11.43 \mathrm{~Hz}), 3.71(1 \mathrm{H}, \mathrm{dd}, \mathrm{J}=6.54,11.43 \mathrm{~Hz}), 7.34-7.35(3 \mathrm{H}, \mathrm{m}), 7.52-7.54(2 \mathrm{H}, \mathrm{m}) .{ }^{13} \mathrm{C} \mathrm{NMR}(101 \mathrm{MHz}$, $\mathrm{CDCl}_{3}$ ) 8: $0.00,0.08,12.65,15.59,18.47,24.37,26.24,30.81,33.56,35.35,65.72,129.22,130.27,135.08$, 141.52. ${ }^{29} \mathrm{Si} \mathrm{NMR}\left(80 \mathrm{MHz}, \mathrm{CDCl}_{3}\right)$ 8: -4.78. HRMS (APCl): $\mathrm{m} / \mathrm{z}$ calculated for $\mathrm{C}_{18} \mathrm{H}_{29} \mathrm{Si}[\mathrm{M}-(\mathrm{OH})]^{+}: 273.2033$, found 273.2031 .

(2-allyl-3-butyl-2-(dimethyl(phenyl)silyl)-3-ethylcyclopropyl)methanol (12e)

Scale, physical description, yield: $0.5 \mathrm{mmol}$, colorless oil. $75 \%$ yield, $124 \mathrm{mg}$. Flash chromatography: 5 to $10 \%$ EtOAc / petroleum ether. ${ }^{1} \mathbf{H}$ NMR $\left(400 \mathbf{~ M H z} \mathrm{CDCl}_{3}\right) \delta: 0.37(3 \mathrm{H}, \mathrm{s}), 0.39(3 \mathrm{H}, \mathrm{s}), 0.82(3 \mathrm{H}, \mathrm{t}, \mathrm{J}=7.06$ $\mathrm{Hz}), 0.90(3 \mathrm{H}, \mathrm{t}, \mathrm{J}=7.36 \mathrm{~Hz}), 1.04(1 \mathrm{H}, \mathrm{dd}, \mathrm{J}=6.8 \mathrm{~Hz}, 8.26 \mathrm{~Hz}), 1.17-1.25(6 \mathrm{H}, \mathrm{m}), 1.47-1.55(2 \mathrm{H}, \mathrm{m}), 2.25-$ $2.27(2 \mathrm{H}, \mathrm{m}), 3.68(1 \mathrm{H}, \mathrm{dd}, \mathrm{J}=8.40,11.49 \mathrm{~Hz}), 3.80(1 \mathrm{H}, \mathrm{dd}, \mathrm{J}=6.74,11.51 \mathrm{~Hz}), 4.89-4.97(2 \mathrm{H}, \mathrm{m}), 5.68-5.76$ $(1 \mathrm{H}, \mathrm{m}), 7.32-7.34(3 \mathrm{H}, \mathrm{m}), 7.52-7.54(2 \mathrm{H}, \mathrm{m}) .{ }^{13} \mathrm{C}$ NMR (101 MHz, $\left.\mathrm{CDCl}_{3}\right)$ 8: $-0.39,0.04,11.25,14.25$, 19.48, 20.35, 23.08, 29.26, 31.88, 33.59, 33.69, 35.05, 60.12, 115.73, 127.78, 128.74, 134.42, 139.03, 140.45. ${ }^{29}$ Si NMR (80 MHz, $\mathrm{CDCl}_{3}$ ) 8: 0.54. HRMS (APCl): m/z calculated for $\mathrm{C}_{21} \mathrm{H}_{33} \mathrm{OSi}[\mathrm{M}-\mathrm{H}]^{+}: 329.2295$, found 329.2299 . 
Scale, physical description, yield: $0.5 \mathrm{mmol}$, colorless oil. $69 \%$ yield, $116 \mathrm{mg}$. Flash chromatography: 5 to $10 \%$ EtOAc / petroleum ether. ${ }^{1} \mathrm{H}$ NMR $\left(400 \mathrm{MHz}, \mathrm{CDCl}_{3}\right) \mathbf{\delta}:-0.45(1 \mathrm{H}, \mathrm{d}, \mathrm{J}=7.33 \mathrm{~Hz}), 0.29(3 \mathrm{H}, \mathrm{s}), 0.32(3 \mathrm{H}$, s), $0.83(3 \mathrm{H}, \mathrm{t}, \mathrm{J}=7.05 \mathrm{~Hz}), 0.88(3 \mathrm{H}, \mathrm{t}, \mathrm{J}=6.99 \mathrm{~Hz}), 0.98-1.02(2 \mathrm{H}, \mathrm{m}), 1.19-1.30(10 \mathrm{H}, \mathrm{m}), 1.51-1.65(3 \mathrm{H}, \mathrm{m})$, $3.58(1 \mathrm{H}, \mathrm{dd}, \mathrm{J}=8.29,11.41 \mathrm{~Hz}), 3.71(1 \mathrm{H}, \mathrm{dd}, \mathrm{J}=6.53,11.41 \mathrm{~Hz}), 7.33-7.35(3 \mathrm{H}, \mathrm{m}), 7.51-7.54(2 \mathrm{H}, \mathrm{m}) .{ }^{13} \mathrm{C}$

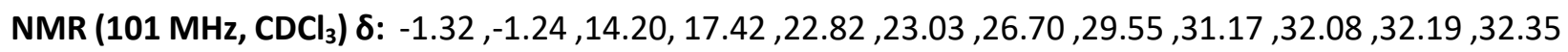

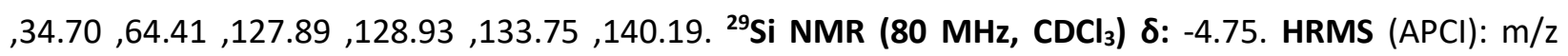
calculated for $\mathrm{C}_{21} \mathrm{H}_{35} \mathrm{Si}[\mathrm{M}-(\mathrm{OH})]^{+}: 315.2508$, found 315.2511 .

(2-butyl-2-decyl-3-(dimethyl(phenyl)silyl)cyclopropyl)methanol (12g)

Scale, physical description, yield: $0.5 \mathrm{mmol}$, colorless oil. $89 \%$ yield, $181 \mathrm{mg}$. Flash chromatography: $5 \%$ EtOAc / petroleum ether. ${ }^{1} \mathrm{H}$ NMR $\left(400 \mathrm{MHz}, \mathrm{CDCl}_{3}\right) \delta:-0.45(1 \mathrm{H}, \mathrm{d}, \mathrm{J}=7.28 \mathrm{~Hz}), 0.29(3 \mathrm{H}, \mathrm{s}), 0.32(3 \mathrm{H}, \mathrm{s})$, $0.83(3 \mathrm{H}, \mathrm{t}, \mathrm{J}=6.92 \mathrm{~Hz}), 0.88(3 \mathrm{H}, \mathrm{t}, \mathrm{J}=6.72 \mathrm{~Hz}), 0.96-1.01(3 \mathrm{H}, \mathrm{m}), 1.19-1.38(2 \mathrm{H}, \mathrm{m}), 1.50-1.55(1 \mathrm{H}, \mathrm{m})$, 1.57-1.65 $(1 \mathrm{H}, \mathrm{m}), 3.55-3.60(1 \mathrm{H}, \mathrm{m}), 3.68-3.73(1 \mathrm{H}, \mathrm{m}), 7.33-7.35(3 \mathrm{H}, \mathrm{m}), 7.51-7.53(2 \mathrm{H}, \mathrm{m}) .{ }^{13} \mathrm{C}$ NMR (101 MHz, $\mathrm{CDCl}_{3}$ ) 8: -1.31, -1.25, 14.19, 14.26, 17.41, 22.82, 23.03, 27.04, 29.49, 29.55, 29.77, 29.80, $29.82,30.17,31.16,32.05,32.08,32.23,34.71,64.40,127.88,128.92,133.75,140.18 .{ }^{29}$ Si NMR (80 MHz, $\mathrm{CDCl}_{3}$ ) ठ: -4.75. HRMS (APCl): m/z calculated for $\mathrm{C}_{26} \mathrm{H}_{45} \mathrm{Si}[\mathrm{M}-(\mathrm{OH})]^{+}: 385.3285$, found 385.3299 .

(2-butyl-2-decyl-3-(dimethyl(phenyl)silyl)-3-iodocyclopropyl)methanol (12h)

Scale, physical description, yield: $0.5 \mathrm{mmol}$, yellow oil. $58 \%$ yield, $153 \mathrm{mg}$. Flash chromatography: $5 \%$ EtOAc / petroleum ether. ${ }^{1} \mathbf{H}$ NMR $\left(400 \mathrm{MHz}, \mathrm{CDCl}_{3}\right)$ 8: $0.54(3 \mathrm{H}, \mathrm{s}), 0.55(3 \mathrm{H}, \mathrm{s}), 0.74(3 \mathrm{H}, \mathrm{t}, \mathrm{J}=12.97 \mathrm{~Hz})$, $0.88(3 \mathrm{H}, \mathrm{t}, \mathrm{J}=6.80 \mathrm{~Hz}), 0.99-1.09(3 \mathrm{H}, \mathrm{m}), 1.21-1.31(18 \mathrm{H}, \mathrm{m}), 1.39-1.47(2 \mathrm{H}, \mathrm{m}), 1.66-1.73(2 \mathrm{H}, \mathrm{m}), 3.69$ $(1 \mathrm{H}, \mathrm{dd}, \mathrm{J}=8.56,11.81 \mathrm{~Hz}), 3.71(1 \mathrm{H}, \mathrm{dd}, \mathrm{J}=5.64,11.81 \mathrm{~Hz}), 7.34-7.36(3 \mathrm{H}, \mathrm{m}), 7.55-7.57(2 \mathrm{H}, \mathrm{m}) .{ }^{13} \mathrm{C}$ NMR (101 MHz, $\mathrm{CDCl}_{3}$ ) 8: -0.75, 1.30, 14.07, 14.28, 22.78, 22.83, 22.57, 27.41, 29.46, 29.50, 29.76, 29.77, 29.78, $30.02,32.06,32.74,33.02,33.37,35.40,65.88,127.95,129.34,134.43,138.88 .{ }^{29} \mathrm{Si} \mathrm{NMR}\left(80 \mathrm{MHz}, \mathrm{CDCl}_{3}\right.$ ) ס: 0.3. HRMS (APCl): $\mathrm{m} / \mathrm{z}$ calculated for $\mathrm{C}_{26} \mathrm{H}_{43} \mathrm{OSi}\left[\mathrm{M}-\left(\mathrm{H}_{2} \mathrm{l}\right)\right]^{+}: 399.3078$, found 399.3093 .

\section{(2,3-diallyl-2-butyl-3-(dimethyl(phenyl)silyl)cyclopropyl)methanol (12i)}

Scale, physical description, yield: $0.5 \mathrm{mmol}$, colorless oil. $62 \%$ yield, $107 \mathrm{mg}$. Flash chromatography: 5 to $10 \%$ EtOAc / petroleum ether. ${ }^{1} \mathrm{H}$ NMR $\left(400 \mathrm{MHz}^{\mathrm{CDCl}}\right.$ ) $\delta: 0.37(3 \mathrm{H}, \mathrm{s}), 0.40(3 \mathrm{H}, \mathrm{s}), 0.82(3 \mathrm{H}, \mathrm{t}, \mathrm{J}=7.17$ 
$\mathrm{Hz}), 1.14-1.31(7 \mathrm{H}, \mathrm{m}), 2.20-2.36(4 \mathrm{H}, \mathrm{m}), 3.73(1 \mathrm{H}, \mathrm{dd}, \mathrm{J}=7.85,11.61 \mathrm{~Hz}), 3.80(1 \mathrm{H}, \mathrm{dd}, \mathrm{J}=7.19,11.63 \mathrm{~Hz})$, 4.90-5.09 (4H, m), 5.67-5.84 (2H, m), 7.33-7.34 (3H, m), 7.52-7.54 (2H, m). $\left.{ }^{13} \mathrm{C} \mathrm{NMR} \mathrm{(101} \mathrm{MHz,} \mathrm{CDCl}{ }_{3}\right)$ 8: $0.52,0.01,14.26,20.32,23.01,29.20,32.00,32.02$, 32.30, 33.13, 35.81, 60.15, 115.98, 116.50, 127.81, 128.83, 134.42, 137.05, 138.69, 140.18. ${ }^{29}$ Si NMR (80 MHz, CDCl $_{3}$ ) 8: 0.64. HRMS (APCI): m/z calculated for $\mathrm{C}_{22} \mathrm{H}_{33} \mathrm{OSi}[\mathrm{M}-\mathrm{H}]^{+}$: 341.2295, found 341.2298.

(3-(dimethyl(phenyl)silyl)-2-ethyl-2-(3-((triisopropylsilyl)oxy)propyl)cyclopropyl)methanol (12j)

Scale, physical description, yield: $0.5 \mathrm{mmol}$, colorless oil. $74 \%$ yield, $166 \mathrm{mg}$. Flash chromatography: 5 to $10 \%$ EtOAc / petroleum ether. ${ }^{1} \mathrm{H}$ NMR $\left(400 \mathrm{MHz} \mathrm{CDCl}_{3}\right) \delta:-0.45(1 \mathrm{H}, \mathrm{d}, \mathrm{J}=7.37 \mathrm{~Hz}), 0.29(3 \mathrm{H}, \mathrm{s}), 0.32(3 \mathrm{H}$, s), $0.93(3 \mathrm{H}, \mathrm{t}, \mathrm{J}=7.27 \mathrm{~Hz}), 1.04-1.06(23 \mathrm{H}, \mathrm{m}), 1.46-1.53(1 \mathrm{H}, \mathrm{m}), 1.59-1.69(3 \mathrm{H}, \mathrm{m}), 3.56-3.61(3 \mathrm{H}, \mathrm{m}), 3.71$ $(1 \mathrm{H}, \mathrm{dd}, \mathrm{J}=6.55,11.35 \mathrm{~Hz}), 7.33-7.35(3 \mathrm{H}, \mathrm{m}), 7.52-.7 .54(2 \mathrm{H}, \mathrm{m}) .{ }^{13} \mathrm{C}$ NMR (101 MHz, CDCl $)$ 8: -1.43, 1.23, 11.26, 12.10, 17.20, 18.19, 24.97, 30.70, 31.86, 32.43, 63.50, 34.33, 127.90, 128.93, 133.71, 140.23.

${ }^{29}$ Si NMR (80 MHz, $\mathrm{CDCl}_{3}$ ) 8: $-4.75,12.31$. HRMS (APCl): $\mathrm{m} / \mathrm{z}$ calculated for $\mathrm{C}_{26} \mathrm{H}_{47} \mathrm{OSi}_{2}[\mathrm{M}-(\mathrm{OH})]^{+}: 431.3165$, found 431.3155 .

(2-allyl-2-(dimethyl(phenyl)silyl)-3-propyl-3-(3-((triisopropylsilyl)oxy)propyl)cyclopropyl)methanol (12k)

Scale, physical description, yield: $0.5 \mathrm{mmol}$, colorless oil. $93 \%$ yield, $234 \mathrm{mg}$.Flash chromatography: 5 to $10 \%$ EtOAc / petroleum ether. ${ }^{1} \mathbf{H}$ NMR $\left(400 \mathrm{MHz}, \mathrm{CDCl}_{3}\right) \delta: 0.36(3 \mathrm{H}, \mathrm{s}), 0.40(3 \mathrm{H}, \mathrm{s}), 0.88(3 \mathrm{H}, \mathrm{t}, \mathrm{J}=6.95$ $\mathrm{Hz}), 1.04-1.06(21 \mathrm{H}, \mathrm{m}), 1.23-1.29(2 \mathrm{H}, \mathrm{m}), 1.23-1.29(2 \mathrm{H}, \mathrm{m}), 1.33-1.37(2 \mathrm{H}, \mathrm{m}), 1.42-1.49(3 \mathrm{H}, \mathrm{m}), 1.58-$ $1.63(2 \mathrm{H}, \mathrm{m}), 2.24-2.26(2 \mathrm{H}, \mathrm{m}), 3.52-3.55(3 \mathrm{H}, \mathrm{m}), 3.80(1 \mathrm{H}, \mathrm{dd}, \mathrm{J}=6.73 \mathrm{~Hz}, 11.53 \mathrm{~Hz}), 4.88-4.96(2 \mathrm{H}, \mathrm{m})$, 5.66-5.76 (1H, m), 7.32-7.33 (3H, m), 7.52-.7.54 (2H, m). $\left.{ }^{13} \mathrm{C} \mathrm{NMR} \mathrm{(101} \mathrm{MHz,} \mathrm{CDCl}\right)$ 8: -0.44, 0.18, 12.10, 14.80, 18.18, 20.07, 20.42, 29.45, 30.58, 32.02, 32.33, 32.63, 33.50, 60.14, 63.68, 115.68, 127.77, 128.76, 134.39, 138.89, 14.042. ${ }^{29} \mathrm{Si}$ NMR (80 $\mathrm{MHz} \mathrm{CDCl}_{3}$ ) $\delta: 0.64,12.43$. HRMS (APCl): $\mathrm{m} / \mathrm{z}$ calculated for $\mathrm{C}_{30} \mathrm{H}_{55} \mathrm{O}_{2} \mathrm{Si}_{2}[\mathrm{M}+\mathrm{H}]^{+}:$503.3735, found 503.3752.

(2-butyl-3-(dimethyl(phenyl)silyl)-2-(3-((triisopropylsilyl)oxy)propyl)cyclopropyl)methanol (12l)

Scale, physical description, yield: $0.5 \mathrm{mmol}$, colorless oil. $74 \%$ yield, $177 \mathrm{mg}$. Flash chromatography: 5 to $10 \%$ EtOAc / petroleum ether. ${ }^{1} \mathrm{H}$ NMR $\left(400 \mathrm{MHz}, \mathrm{CDCl}_{3}\right)$ 8: $-0.43(1 \mathrm{H}, \mathrm{d}, \mathrm{J}=7.37 \mathrm{~Hz}), 0.29(3 \mathrm{H}, \mathrm{s}), 0.32(3 \mathrm{H}$, s), $0.88(3 \mathrm{H}, \mathrm{t}, \mathrm{J}=6.99 \mathrm{~Hz}), 1.03-1.06(25 \mathrm{H}, \mathrm{m}), 1.26-1.30(4 \mathrm{H}, \mathrm{m}), 1.48-1.53(1 \mathrm{H}, \mathrm{m}), 1.62-1.67(3 \mathrm{H}, \mathrm{m})$, 
3.56-3.62 (3H, m), $3.71(1 \mathrm{H}, \mathrm{dd}, \mathrm{J}=6.57 \mathrm{~Hz}, 11.41 \mathrm{~Hz}), 7.33-7.35(3 \mathrm{H}, \mathrm{m}), 7.52-7.5(2 \mathrm{H}, \mathrm{m}) .{ }^{13} \mathrm{C}$ NMR $(101$ $\mathrm{MHz}, \mathrm{CDCl}_{3}$ ) $\delta:-1.41,14.26,17.46,18.19,23.21,29.22,30.76,31.39,32.04,32.08,63.50,64.35,127.90$, 128.93, 133.72, 140.24. ${ }^{29} \mathrm{Si} \mathrm{NMR} \mathrm{(80} \mathrm{MHz,} \mathrm{CDCl}_{3}$ ) 8: -4.72, 12.34. HRMS (APCl): m/z calculated for $\mathrm{C}_{21} \mathrm{H}_{43} \mathrm{Si}$ $[\mathrm{M}-(\mathrm{OH})]^{+}:$459.3478, found 459.3480 .

(3-(dimethyl(phenyl)silyl)-2-(hept-6-en-1-yl)-2-(3-((triisopropylsilyl)oxy)propyl)cyclopropyl)methanol (12m)

Scale, physical description, yield: $0.5 \mathrm{mmol}$, colorless oil. $63 \%$ yield, $165 \mathrm{mg}$.Flash chromatography: 5 to $10 \%$ EtOAc / petroleum ether. ${ }^{1} \mathrm{H}$ NMR $\left(400 \mathrm{MHz}, \mathrm{CDCl}_{3}\right) \delta:-0.43(1 \mathrm{H}, \mathrm{d}, \mathrm{J}=7.37 \mathrm{~Hz}), 0.29(3 \mathrm{H}, \mathrm{s}), 0.33(3 \mathrm{H}, \mathrm{s})$, 1.03-1.07 $(22 \mathrm{H}, \mathrm{m}), 1.22-1.34(4 \mathrm{H}, \mathrm{m}), 1.38-1.55(5 \mathrm{H}, \mathrm{m}), 1.60-1.70(3 \mathrm{H}, \mathrm{m}), 2.03(2 \mathrm{H}, \mathrm{q}, \mathrm{J}=7.07 \mathrm{~Hz}), 3.56-3.62$ $(3 \mathrm{H}, \mathrm{m}), 3.71(1 \mathrm{H}, \mathrm{dd}, \mathrm{J}=6.52 \mathrm{~Hz}, 11.41 \mathrm{~Hz}), 4.92-5.02(2 \mathrm{H}, \mathrm{m}), 5.76-5.86(1 \mathrm{H}, \mathrm{m}), 7.34-7.35(3 \mathrm{H}, \mathrm{m}), 7.52-7.55$ (2H, m). ${ }^{13}$ C NMR (101 MHz, CDCl $)$ ): -1.41 -1.23, 12.10, 17.49, 18.18, 26.81, 29.07, 29.59, 30.71, 30.74, 31.38, $32.02,32.27,33.91,63.47,64.29,114.36,127.89,128.93,133.70,139.21,140.19 .{ }^{29} \mathrm{Si}$ NMR (80 MHz, CDCl3)

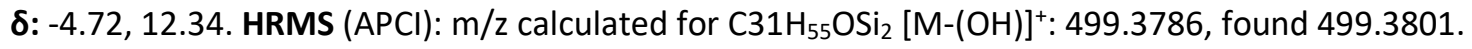

(3-(dimethyl(phenyl)silyl)-2-phenethyl-2-(3-((triisopropylsilyl)oxy)propyl)cyclopropyl)methanol (12n)

Scale, physical description, yield: $0.5 \mathrm{mmol}$, colorless oil. $66 \%$ yield, $174 \mathrm{mg}$. Flash chromatography: 5 to $10 \%$ EtOAc / petroleum ether. ${ }^{1} \mathrm{H}$ NMR $\left(400 \mathrm{MHz} \mathrm{CDCl}_{3}\right) \delta:-0.35(1 \mathrm{H}, \mathrm{d}, \mathrm{J}=7.41 \mathrm{~Hz}), 0.33(3 \mathrm{H}, \mathrm{s}), 0.35(3 \mathrm{H}, \mathrm{s})$, 1.06-1.14 $(22 \mathrm{H}, \mathrm{m}), 1.24-1.28(1 \mathrm{H}, \mathrm{m}), 1.37-1.44(1 \mathrm{H}, \mathrm{m}), 1.57-1.62(1 \mathrm{H}, \mathrm{m}), 1.67-1.80(2 \mathrm{H}, \mathrm{m}), 1.96(1 \mathrm{H}, \mathrm{dt}, \mathrm{J}$ $=4.92 \mathrm{~Hz}, 18.52 \mathrm{~Hz}), 2.58(1 \mathrm{H}, \mathrm{dt}, \mathrm{J}=4.76 \mathrm{~Hz}, 12.47 \mathrm{~Hz}), 2.80(1 \mathrm{H}, \mathrm{dt}, \mathrm{J}=4.72 \mathrm{~Hz}, 19.12 \mathrm{~Hz}), 3.58(1 \mathrm{H}, \mathrm{dd}, \mathrm{J}=8.35$ $\mathrm{Hz}, 11.39 \mathrm{~Hz}), 3.66(2 \mathrm{H}, \mathrm{t}, \mathrm{J}=5.97 \mathrm{~Hz}), 3.74(1 \mathrm{H}, \mathrm{dd}, \mathrm{J}=6.45 \mathrm{~Hz}, 11.41 \mathrm{~Hz}), 7.16-7.21(3 \mathrm{H}, \mathrm{m}), 7.27-7.30(2 \mathrm{H}, \mathrm{m})$, 7.36-7.38 (3H, m), 7.55-7.60 (2H, m). ${ }^{13} \mathrm{C}$ NMR (101 MHz, $\left.\mathrm{CDCl}_{3}\right)$ 8: $-1.46-1.23,12.09,17.46,18.20,30.73$, $30.77,31.34,32.27,33.50,34.87,63.40,64.15,125.89,127.93,128.41,128.50,128.99,133.70,140.03,142.68$.

${ }^{29} \mathrm{Si}$ NMR (80 MHz, $\mathrm{CDCl}_{3}$ ) $\delta:-4.68,12.46$. HRMS (APCl): m/z calculated for $\mathrm{C}_{32} \mathrm{H}_{51} \mathrm{OSi}_{2}[\mathrm{M}-(\mathrm{OH})]^{+}: 507.3473$, found 507.3473 .

\section{2-butyl-3-(hydroxymethyl)-2-methyl-1-(trimethylsilyl)cyclopropanecarboxylic acid (120)}

Scale, physical description, yield: $0.5 \mathrm{mmol}$, white solid. $73 \%$ yield, $95 \mathrm{mg}$. Flash chromatography: $40 \%$

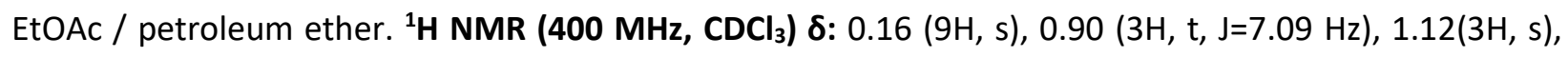
1.25-1.37 (5H, m), 1.43-1.47 (1H, m), 1.59-1.66 (1H, m), $3.64(1 \mathrm{H}, \mathrm{dd}, \mathrm{J}=10.43,12.11 \mathrm{~Hz}), 3.94(1 \mathrm{H}, \mathrm{dd}$, J=6.13, $12.13 \mathrm{~Hz}), 7.35(3 \mathrm{H}, \mathrm{m}), 7.53(2 \mathrm{H}, \mathrm{m}) .{ }^{13} \mathrm{C}$ NMR (101 MHz, $\left.\mathrm{CDCl}_{3}\right)$ 8: -0.47, 14.23, 16.72, 22.87, 
29.34, 31.27, 32.31, 34.40, 38.50, 60.81, 179.47. ${ }^{29}$ Si NMR (80 MHz, $\mathrm{CDCl}_{3}$ ) 8: 3.58. HRMS (APCl): $\mathrm{m} / \mathrm{z}$ calculated for $\mathrm{C}_{13} \mathrm{H}_{27} \mathrm{O}_{3} \mathrm{Si}[\mathrm{M}+\mathrm{H}]^{+}: 259.1724$, found 259.1741 .

\section{(2-butyl-2-decyl-3-(trimethylsilyl)cyclopropyl)methanol (12p)}

Scale, physical description, yield: $0.5 \mathrm{mmol}$, colorless oil. $75 \%$ yield, $128 \mathrm{mg}$. Flash chromatography: $5 \%$ EtOAc / petroleum ether. ${ }^{1} \mathrm{H}$ NMR $\left(400 \mathrm{MHz}, \mathrm{CDCl}_{3}\right) \delta:-0.68(1 \mathrm{H}, \mathrm{d}, \mathrm{J}=7.32 \mathrm{~Hz}), 0.02(9 \mathrm{H}, \mathrm{s}), 0.86-0.91(6 \mathrm{H}$, m), 1.25-1.29 $(21 \mathrm{H}, \mathrm{m}), 1.39-1.42(2 \mathrm{H}, \mathrm{m}), 1.59-165(1 \mathrm{H}, \mathrm{m}), 3.54(1 \mathrm{H}, \mathrm{dd}, \mathrm{J}=8.47,11.39 \mathrm{~Hz}), 3.69(1 \mathrm{H}, \mathrm{dd}$, $\mathrm{J}=6.39,11.43 \mathrm{~Hz}) .{ }^{13} \mathrm{C}$ NMR (101 MHz, $\mathrm{CDCl}_{3}$ ) $\delta: 0.12,14.26,14.30,17.95,22.83,23.16,27.07,29.50$, $29.66,29.69,29.79,29.85,30.18,30.79,31.82,32.06,32.16,34.72,64.54 .{ }^{29} \mathrm{Si} \mathrm{NMR}\left(80 \mathrm{MHz}, \mathrm{CDCl}_{3}\right)$ 8: 0.21. HRMS (APCl): $\mathrm{m} / \mathrm{z}$ calculated for $\mathrm{C}_{21} \mathrm{H}_{43} \mathrm{Si}[\mathrm{M}-(\mathrm{OH})]^{+}: 323.3129$, found 323.3171 .

\section{(2-butyl-2-(3-phenylpropyl)-3-(trimethylsilyl)cyclopropyl)methanol (12q)}

Scale, physical description, yield: $0.5 \mathrm{mmol}$, colorless oil. $94 \%$ yield, $151 \mathrm{mg}$. Flash chromatography: 5 to $10 \%$ EtOAc / petroleum ether. ${ }^{1} \mathrm{H}$ NMR $\left(400 \mathrm{MHz}, \mathrm{CDCl}_{3}\right)$ 8: $-0.68(1 \mathrm{H}, \mathrm{d}, \mathrm{J}=7.33 \mathrm{~Hz}), 0.03(9 \mathrm{H}, \mathrm{s}), 0.88(3 \mathrm{H}$, t, J=7.00 Hz), 1.03-1.10 (2H, m), 1.24-1.29 (3H, m), 1.33-1.38 $(1 \mathrm{H}, \mathrm{m}), 1.59-1.67(3 \mathrm{H}, \mathrm{m}), 1.75-1.81(1 \mathrm{H}$, m), 2.57-2.65 (2H, m), $3.50(1 \mathrm{H}, \mathrm{dd}, \mathrm{J}=8.43,11.35 \mathrm{~Hz}), 3.66(1 \mathrm{H}, \mathrm{dd}, \mathrm{J}=6.40,11.45 \mathrm{~Hz}), 7.18-7.20(3 \mathrm{H}, \mathrm{m})$, 7.27-7.31 (2H, m). ${ }^{13} \mathrm{C}$ NMR (101 MHz, $\left.\mathrm{CDCl}_{3}\right)$ 8: 0.13, 14.28, 17.91, 23.11, 28.82, 29.59, 30.62, 31.76,

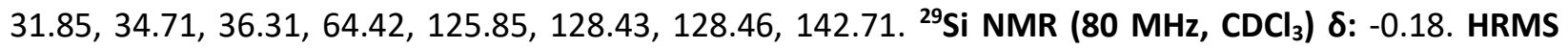
(APCI): $\mathrm{m} / \mathrm{z}$ calculated for $\mathrm{C}_{20} \mathrm{H}_{33} \mathrm{Si}[\mathrm{M}-(\mathrm{OH})]^{+}: 301.2346$, found 301.2373 .

\section{(3-(benzyldimethylsilyl)-2-butyl-2-ethylcyclopropyl)methanol (12r).}

Scale, physical description, yield: $0.5 \mathrm{mmol}$, colorless oil. $61 \%$ yield, $93 \mathrm{mg}$. Flash chromatography: 5 to $10 \%$ EtOAc / petroleum ether. ${ }^{1} \mathrm{H}$ NMR $\left(400 \mathrm{MHz}, \mathrm{CDCl}_{3}\right)$ 8: $-0.69(1 \mathrm{H}, \mathrm{d}, \mathrm{J}=7.32 \mathrm{~Hz}),-0.01(3 \mathrm{H}, \mathrm{s}), 0.04(3 \mathrm{H}$, s), $0.88(3 \mathrm{H}, \mathrm{t}, \mathrm{J}=7.00 \mathrm{~Hz}), 0.92(3 \mathrm{H}, \mathrm{t}, \mathrm{J}=7.10 \mathrm{~Hz}), 0.99-1.06(1 \mathrm{H}, \mathrm{m}), 1.24-1.30(4 \mathrm{H}, \mathrm{m}), 1.34-159(2 \mathrm{H}, \mathrm{m})$, 1.60-165 (2H, m), $2.14(2 \mathrm{H}, \mathrm{s}), 3.50-3.53(2 \mathrm{H}, \mathrm{m}), 7.01(2 \mathrm{H}, \mathrm{t}, \mathrm{J}=7.03 \mathrm{~Hz}), 7.08(1 \mathrm{H}, \mathrm{t}, \mathrm{J}=7.38 \mathrm{~Hz}), 7.21(2 \mathrm{H}$, t, J=7.57 Hz). ${ }^{13} \mathrm{C}$ NMR (101 MHz, $\left.\mathrm{CDCl}_{3}\right)$ 8: $-1.84,-1.67,11.31,14.29,16.33,23.11,24.84,27.29,29.49$, 31.61, 32.21, 34.24, 64.37, 124.18, 128.27, 128.34, 140.52. ${ }^{29}$ Si NMR (80 MHz, $\mathrm{CDCl}_{3}$ ) 8: 0.49. HRMS (APCI): $\mathrm{m} / \mathrm{z}$ calculated for $\mathrm{C}_{19} \mathrm{H}_{31} \mathrm{Si}[\mathrm{M}-(\mathrm{OH})]^{+}:$287.2195, found 287.2183. 
(3-(benzyldimethylsilyl)-2-butyl-2-propylcyclopropyl)methanol (12s)

Scale, physical description, yield: $0.5 \mathrm{mmol}$, colorless oil. $48 \%$ yield, $77 \mathrm{mg}$. Flash chromatography: 5 to $10 \%$ EtOAc / petroleum ether. ${ }^{1} \mathrm{H}$ NMR $\left(400 \mathrm{MHz}, \mathrm{CDCl}_{3}\right)$ 8: $-0.66(1 \mathrm{H}, \mathrm{d}, \mathrm{J}=7.29 \mathrm{~Hz}), 0.02(3 \mathrm{H}, \mathrm{s}), 0.04(3 \mathrm{H}$, s), 0.80-0.84 $(2 \mathrm{H}, \mathrm{m}), 0.86-0.92(6 \mathrm{H}, \mathrm{m}), 1.26-1.30(4 \mathrm{H}, \mathrm{m}), 1.38-1.50(2 \mathrm{H}, \mathrm{m}), 1.60-1.66(3 \mathrm{H}, \mathrm{m}), 2.13(2 \mathrm{H}$, s), 3.50-3.52 (2H, m), $7.01(2 \mathrm{H}, \mathrm{d}, \mathrm{J}=7.53 \mathrm{~Hz}), 7.08(1 \mathrm{H}, \mathrm{t}, \mathrm{J}=7.31 \mathrm{~Hz}), 7.21(2 \mathrm{H}, \mathrm{t}, \mathrm{J}=7.45 \mathrm{~Hz}) .{ }^{13} \mathrm{C}$ NMR (101 $\mathrm{MHz}_{2} \mathrm{CDCl}_{3}$ ) $\delta:-1.82,-1.64,14.28,14.67,16.62,20.25,23.11,27.30,29.57,30.46,31.73,34.54,34.95$, $64.39,124.17,128.26,128.33,140.51 .{ }^{29} \mathrm{Si} \mathrm{NMR} \mathrm{(80} \mathrm{MHz,} \mathrm{CDCl}_{3}$ ) 8: 0.52. HRMS (APCI): m/z calculated for $\mathrm{C}_{20} \mathrm{H}_{33} \mathrm{Si}[\mathrm{M}-(\mathrm{OH})]^{+}:$301.2346, found 301.2337.

1-(benzyldimethylsilyl)-2-butyl-3-(hydroxymethyl)-2-methylcyclopropanecarboxylic acid (12t)

Scale, physical description, yield: $0.5 \mathrm{mmol}$, white solid. 57\% yield, $96 \mathrm{mg}$. Flash chromatography: $40 \%$ EtOAc / petroleum ether. ${ }^{1} \mathbf{H}$ NMR (400 $\left.\mathbf{M H z} \mathrm{CDCl}_{3}\right)$ $\delta: 0.07(3 \mathrm{H}, \mathrm{s}), 0.12(3 \mathrm{H}, \mathrm{s}), 0.89(3 \mathrm{H}, \mathrm{t}, \mathrm{J}=6.89 \mathrm{~Hz})$, 1.08-1.11 (1H, m), $1.15(3 \mathrm{H}, \mathrm{s}), 1.26-1.30(3 \mathrm{H}, \mathrm{m}), 1.37-1.48(2 \mathrm{H}, \mathrm{m}), 1.53-1.59(1 \mathrm{H}, \mathrm{m}), 2.28(2 \mathrm{H}, \mathrm{q}, \mathrm{J}=14.00$ $\mathrm{Hz}), 3.69(1 \mathrm{H}, \mathrm{t}, \mathrm{J}=11.17 \mathrm{~Hz}), 3.95(1 \mathrm{H}, \mathrm{dd}, \mathrm{J}=6.11,12.11 \mathrm{~Hz}), 7.01(2 \mathrm{H}, \mathrm{d}, \mathrm{J}=7.65 \mathrm{~Hz}), 7.08(1 \mathrm{H}, \mathrm{t}, \mathrm{J}=7.31$ $\mathrm{Hz}), 7.20(1 \mathrm{H}, \mathrm{t}, \mathrm{J}=7.49 \mathrm{~Hz}) .{ }^{13} \mathrm{C}$ NMR (101 MHz, $\left.\mathrm{CDCl}_{3}\right)$ 8: -2.42, -2.41, 14.22, 16.66, 22.84, 25.35, 29.32, $31.60,31.91,34.52,38.61,60.73,124.53,128.41,128.57,139.13,179.25 .{ }^{29} \mathrm{Si}$ NMR (80 MHz, $\left.\mathrm{CDCl}_{3}\right)$ 8: 3.24. HRMS (APCl): $\mathrm{m} / \mathrm{z}$ calculated for $\mathrm{C}_{19} \mathrm{H}_{31} \mathrm{O}_{3} \mathrm{Si}[\mathrm{M}+\mathrm{H}]^{+}: 335.2037$, found 335.2067.

\section{(2-butyl-2-ethyl-3-(1-methylsiletan-1-yl)cyclopropyl)methanol (12u)}

Scale, physical description, yield: $0.5 \mathrm{mmol}$, colorless oil. $47 \%$ yield, $60 \mathrm{mg}$. Flash chromatography: 5 to $10 \%$ EtOAc / petroleum ether. ${ }^{1} \mathrm{H}$ NMR $\left(400 \mathrm{MHz} \mathrm{CDCl}_{3}\right)$ 8: $-0.49(1 \mathrm{H}, \mathrm{d}, \mathrm{J}=7.17 \mathrm{~Hz}), 0.29(3 \mathrm{H}, \mathrm{S}), 0.87(3 \mathrm{H}$, t, J=7.09 Hz), $0.94(3 \mathrm{H}, \mathrm{t}, \mathrm{J}=7.33 \mathrm{~Hz}), 0.97-1.01(3 \mathrm{H}, \mathrm{m}), 1.09-1.16(2 \mathrm{H}, \mathrm{m}), 1.23-1.37(6 \mathrm{H}, \mathrm{m}), 1.54-1.66$ $(2 \mathrm{H}, \mathrm{m}), 1.97-2.13(2 \mathrm{H}, \mathrm{m}), 3.59(1 \mathrm{H}, \mathrm{dd}, \mathrm{J}=8.27 \mathrm{~Hz}, 11.43 \mathrm{~Hz}), 3.73(1 \mathrm{H}, \mathrm{dd}, \mathrm{J}=6.51 \mathrm{~Hz}, 11.43 \mathrm{~Hz}) .{ }^{13} \mathrm{C} \mathrm{NMR}$ (101 $\mathrm{MHz}, \mathrm{CDCl}_{3}$ ) : : $-0.43,11.33,14.24,14.84,15.08,17.28,18.50,23.06,24.61,29.50,32.37,32.47$, 34.09, 64.22. ${ }^{29} \mathrm{Si} \mathrm{NMR}\left(80 \mathrm{MHz}, \mathrm{CDCl}_{3}\right.$ ) 8: 17.20. HRMS (APCl): m/z calculated for $\mathrm{C}_{14} \mathrm{H}_{27} \mathrm{Si}[\mathrm{M}-(\mathrm{OH})]^{+}$: 223.1877, found 223.1865 .

\section{(2-butyl-3-(1-methylsiletan-1-yl)-2-propylcyclopropyl)methanol (12v)}

Scale, physical description, yield: $0.5 \mathrm{mmol}$, colorless oil. $71 \%$ yield, $90 \mathrm{mg}$. Flash chromatography: 5 to $10 \%$ EtOAc / petroleum ether. ${ }^{1} \mathrm{H}$ NMR $\left(400 \mathrm{MHz}^{\mathrm{CDCl}} \mathrm{CD}_{3}\right) \mathrm{\delta}:-0.45(1 \mathrm{H}, \mathrm{d}, \mathrm{J}=7.13 \mathrm{~Hz}), 0.28(3 \mathrm{H}, \mathrm{S}), 0.86(3 \mathrm{H}$, 
t, J=7.06 Hz), $0.90(3 \mathrm{H}, \mathrm{t}, \mathrm{J}=7.33 \mathrm{~Hz}), 0.96-1.01(4 \mathrm{H}, \mathrm{m}), 1.04-1.10(1 \mathrm{H}, \mathrm{s}), 1.23-1.67(10 \mathrm{H}, \mathrm{m}), 1.96-2.11$ $(2 \mathrm{H}, \mathrm{m}), 3.58(1 \mathrm{H}, \mathrm{dd}, \mathrm{J}=8.29,11.45 \mathrm{~Hz}), 3.72(1 \mathrm{H}, \mathrm{dd}, \mathrm{J}=6.51,11.43 \mathrm{~Hz}) .{ }^{13} \mathrm{C} \mathbf{N M R}\left(101 \mathrm{MHz}, \mathrm{CDCl}_{3}\right)$ 8: 0.41, 14.21, 14.59, 14.84, 15.07, 17.55, 18.48, 20.25, 23.05, 29.54, 31.11, 31.92, 34.24, 34.79, 64.19. ${ }^{29} \mathrm{Si}$ NMR (80 MHz, $\mathrm{CDCl}_{3}$ ) 8: 17.23. HRMS (APCl): m/z calculated for $\mathrm{C}_{15} \mathrm{H}_{29} \mathrm{Si}[\mathrm{M}-(\mathrm{OH})]^{+}: 237.2033$, found 237.2041.

(2-butyl-3-(dimethylsilyl)-2-methyl-1-phenylcyclopropyl)methanol (12w)

Scale, physical description, yield: $0.5 \mathrm{mmol}$, pale-yellow oil. $49 \%$ yield, $68 \mathrm{mg}$. Flash chromatography: 5 to $10 \%$ EtOAc / petroleum ether. ${ }^{1} \mathrm{H}$ NMR $\left(400 \mathrm{MHz} \mathrm{CDCl}_{3}\right) \delta:-0.17(1 \mathrm{H}, \mathrm{d}, \mathrm{J}=4.04 \mathrm{~Hz}), 0.00(3 \mathrm{H}, \mathrm{d}, \mathrm{J}=3.68$ $\mathrm{Hz}), 0.18(3 \mathrm{H}, \mathrm{d}, \mathrm{J}=3.56 \mathrm{~Hz}), 0.80(3 \mathrm{H}, \mathrm{t}, \mathrm{J}=7.29 \mathrm{~Hz}), 0.92-0.99(1 \mathrm{H}, \mathrm{m}), 1.14(2 \mathrm{H}, \mathrm{q}, \mathrm{J}=14.56 \mathrm{~Hz}), 1.28-1.32$ $(2 \mathrm{H}, \mathrm{m}), 1.34(3 \mathrm{H}, \mathrm{s}), 1.44-1.55(1 \mathrm{H}, \mathrm{m}), 3.72(2 \mathrm{H}, \mathrm{q}, \mathrm{J}=21.57 \mathrm{~Hz}), 3.81(1 \mathrm{H}, \mathrm{sex}, \mathrm{J}=3.77 \mathrm{~Hz}), 7.20-7.22(1 \mathrm{H}$, m), 7.28-7.29 (4H, m). ${ }^{13} \mathrm{C}$ NMR (101 MHz, $\left.\mathrm{CDCl}_{3}\right)$ 8: -2.22, -2.02, 14.29, 20.97, 23.06, 23.40, 30.00, 30.11, $36.41,43.47,71.42,126.79,128.24,131.37,140.57 .{ }^{29} \mathrm{Si}$ NMR (80 MHz, $\mathrm{CDCl}_{3}$ ) 8: -18.21. HRMS (APCI): $\mathrm{m} / \mathrm{z}$ calculated for $\mathrm{C}_{17} \mathrm{H}_{27} \mathrm{OSi}[\mathrm{M}+\mathrm{H}]^{+}: 275.1831$, found 275.1857 .

\section{(3-(dimethylsilyl)-2-hexyl-2-methyl-1-phenylcyclopropyl)methanol (12x)}

Scale, physical description, yield: $0.5 \mathrm{mmol}$, pale-yellow oil. $66 \%$ yield, $101 \mathrm{mg}$. Flash chromatography: 5 to $10 \%$ EtOAc / petroleum ether. ${ }^{1} \mathbf{H}$ NMR $\left(400 \mathbf{M H z} \mathbf{C D C l}_{3}\right)$ 8: $-0.14(1 \mathrm{H}, \mathrm{d}, \mathrm{J}=4.35 \mathrm{~Hz}), 0.03(3 \mathrm{H}, \mathrm{d}$, $\mathrm{J}=3.64), 0.21(3 \mathrm{H}, \mathrm{d}, \mathrm{J}=3.61), 0.85(3 \mathrm{H}, \mathrm{t}, \mathrm{J}=6.95 \mathrm{~Hz}), 0.94-1.01(1 \mathrm{H}, \mathrm{m}), 1.12-1.15(2 \mathrm{H}, \mathrm{m}), 1.21-1.26(3 \mathrm{H}$, m), 1.32-1.34 (2H, m), $1.37(3 \mathrm{H}, \mathrm{s}), 1.54-1.58(1 \mathrm{H}, \mathrm{m}), 3.75(2 \mathrm{H}, \mathrm{q}, \mathrm{J}=21.62 \mathrm{~Hz}), 3.85(1 \mathrm{H}, \mathrm{sex}, \mathrm{J}=3.76), 7.23-$ $7.25(1 \mathrm{H}, \mathrm{m}), 7.31-7.32(4 \mathrm{H}, \mathrm{m}) .{ }^{13} \mathrm{C}$ NMR (101 MHz, $\left.\mathrm{CDCl}_{3}\right)$ 8: -2.23, -2.03, 14.17, 20.97, 22.72, 23.06, $27.78,30.02,30.17,32.05,36.78,43.48,71.41,126.78,128.24,131.37,140.52 .{ }^{29} \mathrm{Si} \mathrm{NMR}\left(80 \mathrm{MHz}, \mathrm{CDCl}_{3}\right)$ ס: --18.20. HRMS (APCI): $\mathrm{m} / \mathrm{z}$ calculated for $\mathrm{C}_{19} \mathrm{H}_{31} \mathrm{OSi}[\mathrm{M}-\mathrm{H}]^{+}: 303.2139$, found 303.2104. 
((1R,2S,3S)-2-allyl-3-butyl-2-(dimethylsilyl)-3-methyl-1-phenylcyclopropyl)methanol (12y)

Scale, physical description, yield: $0.5 \mathrm{mmol}$, pale-yellow oil. $59 \%$ yield, $94 \mathrm{mg}$. Flash chromatography: 5 to $10 \%$ EtOAc / petroleum ether. ${ }^{1} \mathrm{H}$ NMR $\left(400 \mathrm{MHz}, \mathrm{CDCl}_{3}\right) \delta:-0.04(3 \mathrm{H}, \mathrm{d}, \mathrm{J}=3.80 \mathrm{~Hz}), 0.19(3 \mathrm{H}, \mathrm{d}, \mathrm{J}=3.68$ $\mathrm{Hz}), 0.87(3 \mathrm{H}, \mathrm{t}, \mathrm{J}=7.295 \mathrm{~Hz}), 1.17-1.22(2 \mathrm{H}, \mathrm{m}), 1.24-1.29(2 \mathrm{H}, \mathrm{m}), 1.33(3 \mathrm{H}, \mathrm{s}), 1.54-1.60(2 \mathrm{H}, \mathrm{m}), 2.51-$ $2.54(2 \mathrm{H}, \mathrm{m}), 3.62(1 \mathrm{H}, \mathrm{sep}, \mathrm{J}=3.66), 3.86(2 \mathrm{H}, \mathrm{q}, \mathrm{J}=12.71), 5.03-5.14(2 \mathrm{H}, \mathrm{m}), 5.87-5.97(1 \mathrm{H}, \mathrm{m}), 7.22-7.25$ (1H, m), 7.31-7.34 (4H, m). ${ }^{13} \mathrm{C}$ NMR (101 MHz, $\left.\mathrm{CDCl}_{3}\right)$ 8: -1.94, -1.26, 14.33, 15.27, 23.44, 24.25, 30.23, $32.35,33.21,37.50,44.42,66.26,115.82,126.73,128.19,131.65,138.97,141.33 .{ }^{29}$ Si NMR (80 MHz, $\mathrm{CDCl}_{3}$ ) $\delta:$ 1.75. HRMS (APCI): $\mathrm{m} / \mathrm{z}$ calculated for $\mathrm{C}_{20} \mathrm{H}_{31} \mathrm{OSi}[\mathrm{M}+\mathrm{H}]^{+}: 315.2114$, found 315.2128.

HPLC: The racemic and chiral examples were analyzed using a CHIRALCEL ${ }^{\circledR} A S-H$ column $(0.46 \mathrm{~cm} \emptyset \times 25 \mathrm{~cm})$, $n$-hexane/isopropanol 95/5, 0.5mL/min, $214 n m . t_{R}($ major $)=8.68 \mathrm{~min}, t_{R}(\operatorname{minor})=9.71 \mathrm{~min}$.
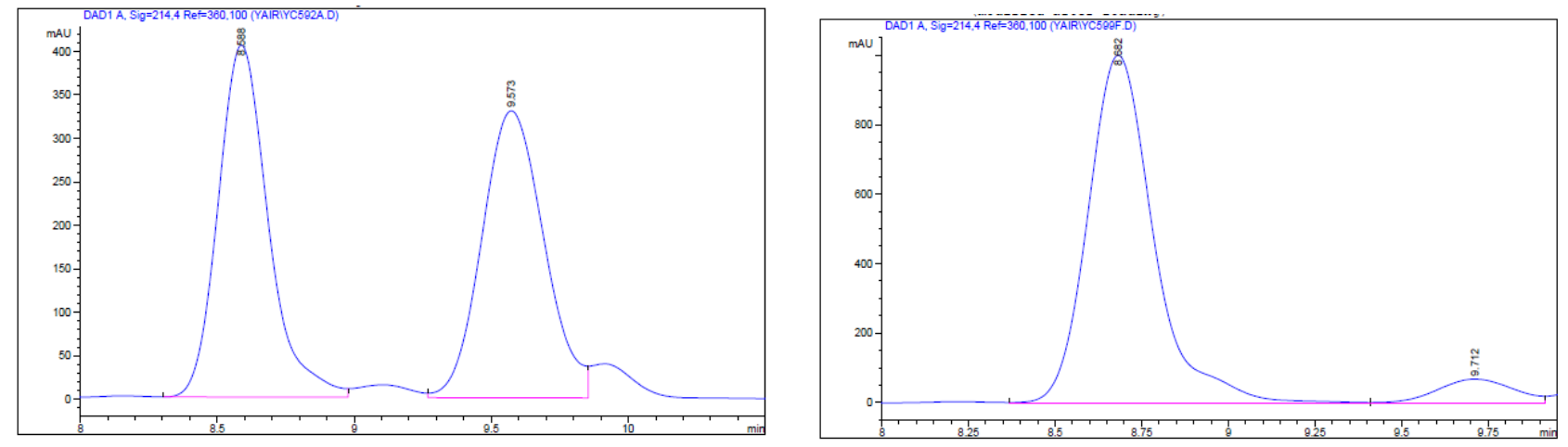

Signal 1: DAD1 A, Sig $=214,4$ Ref $=360,100$

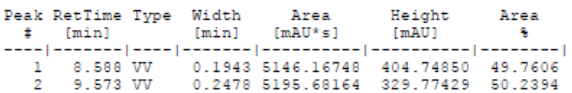

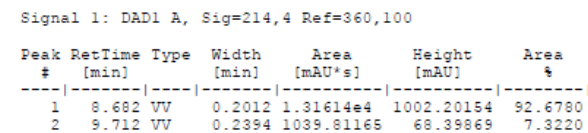

\section{(3-(dimethylsilyl)-2-hexyl-1,2-dimethylcyclopropyl)methanol (12z)}

Scale, physical description, yield: $0.5 \mathrm{mmol}$, Colorless oil. $81 \%$ yield, $99 \mathrm{mg}$. Flash chromatography: 5 to $10 \%$ EtOAc / petroleum ether. ${ }^{1} \mathbf{H}$ NMR $\left(400 \mathrm{MHz}, \mathrm{CDCl}_{3}\right) \delta:-0.61(1 \mathrm{H}, \mathrm{d}, \mathrm{J}=6.13 \mathrm{~Hz}), 0.09(3 \mathrm{H}, \mathrm{d}, \mathrm{J}=3.64$ $\mathrm{Hz}), 0.12(3 \mathrm{H}, \mathrm{d}, \mathrm{J}=3.64 \mathrm{~Hz}), 0.88(3 \mathrm{H}, \mathrm{t}, \mathrm{J}=6.77 \mathrm{~Hz}), 1.20(3 \mathrm{H}, \mathrm{s}), 1.25(3 \mathrm{H}, \mathrm{s}), 1.27-1.47(10 \mathrm{H}, \mathrm{m}), 3.48(1 \mathrm{H}$, d, J=11.29 Hz), $3.63(1 \mathrm{H}, \mathrm{d}, \mathrm{J}=11.25 \mathrm{~Hz}), 3.86-3.93(1 \mathrm{H}, \mathrm{m}) .{ }^{13} \mathrm{C} \mathbf{N M R}\left(101 \mathrm{MHz}, \mathrm{CDCl}_{3}\right)$ 8: -2.71, 14.24, $15.38,21.50,21.93,22.82,27.61,29.45,30.02,31.69,32.06,34.58,71.27 .{ }^{29}$ Si NMR (80 MHz, $\left.\mathrm{CDCl}_{3}\right)$ 8: 19.85. HRMS $(A P C l): m / z$ calculated for $\mathrm{C}_{14} \mathrm{H}_{29} \mathrm{OSi}[\mathrm{M}+\mathrm{H}]^{+}: 241.1982$, found 241.1990 . 
4. General procedure for the reduction of cyclopropenylsilanes ${ }^{1}$

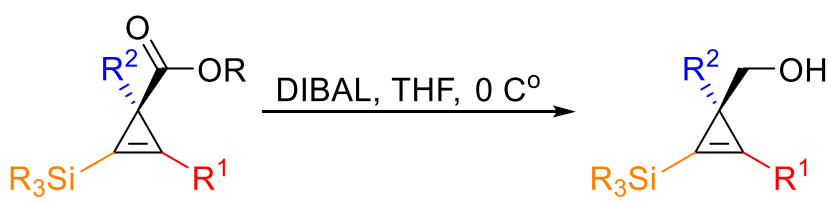

In a flame dried three necked flask equipped with addition funnel, cyclopropenylsilane ester (1 eq.) was dissolved in THF (1M) and cooled to $0^{\circ} \mathrm{C}$. DIBAL (2.2 eq., $1 \mathrm{M}$ in hexane) was added dropwise from the addition funnel. Reaction mixture was stirred at $0^{\circ} \mathrm{C}$ until full consumption of cyclopropenylsilane ester (followed by TLC, $15 \%$ EtOAc/ petroleum ether, $p$-anisaldehyde/ $\mathrm{KMnO}_{4}$, ca. $1 \mathrm{hr}$ ). The reaction was diluted with $\mathrm{Et}_{2} \mathrm{O}$. Solid Rochelle salt was added, and reaction was diluted with water. The mixture was stirred until two separated phases appeared. The aqueous layer was extracted three times with $\mathrm{Et}_{2} \mathrm{O}$, and the combined organic phases were dried over $\mathrm{Na}_{2} \mathrm{SO}_{4}$, filtered, and concentrated under reduced pressure. Crude mixture was then purified by flash chromatography using EtOAc / petroleum ether as eluent

\section{(2-butyl-3-(dimethyl(phenyl)silyl)cycloprop-2-en-1-yl)methanol (11a)}

Scale, physical description, yield: $20 \mathrm{mmol}$, pale-yellow oil. $72 \%$ yield, 3.78 gr. Flash chromatography: 5 to $10 \%$ EtOAc / petroleum ether. ${ }^{1} \mathrm{H}$ NMR $\left(400 \mathrm{MHz}, \mathrm{CDCl}_{3}\right) \delta: 0.47(6 \mathrm{H}, \mathrm{d}, \mathrm{J}=1.40 \mathrm{~Hz}), 0.92(3 \mathrm{H}, \mathrm{t}, \mathrm{J}=$ $7.33 \mathrm{~Hz}), 1.37(2 \mathrm{H}$, sex, J = $7.39 \mathrm{~Hz}), 1.56(2 \mathrm{H}$, quin, J = $7.51 \mathrm{~Hz}), 1.66(1 \mathrm{H}, \mathrm{t}, \mathrm{J}=4.74 \mathrm{~Hz}), 2.59(2 \mathrm{H}, \mathrm{t}, \mathrm{J}=$ $7.33 \mathrm{~Hz}), 3.41(1 \mathrm{H}, \mathrm{dd}, \mathrm{J}=5.15 \mathrm{~Hz}, 10.75 \mathrm{~Hz}), 3.53(1 \mathrm{H}, \mathrm{dd}, \mathrm{J}=4.34 \mathrm{~Hz}, 10.75 \mathrm{~Hz}), 7.38-7.40$ (3H, m), 7.56$7.59(2 \mathrm{H}, \mathrm{m}) .{ }^{13} \mathrm{C}$ NMR $\left(101 \mathrm{MHz}, \mathrm{CDCl}_{3}\right)$ 8: -2.17, -1.95, 13.88, 22.42, 22.50, 27.89, 29.61, 69.66, 109.16, 128.06, 129.44, 133.69, 137.76, 141.44. ${ }^{29}$ Si NMR (80 MHz, $\mathrm{CDCl}_{3}$ ) 8: -15.58. HRMS (APCI): m/z calculated for $\mathrm{C}_{16} \mathrm{H}_{25} \mathrm{OSi}[\mathrm{M}+\mathrm{H}]^{+}:$261.1669, found 261.1697.

\section{(2-(benzyldimethylsilyl)-3-butylcycloprop-2-en-1-yl)methanol (11c)}

Scale, physical description, yield: $5 \mathrm{mmol}$, pale-yellow oil. $68 \%$ yield, 0.93 gr. Flash chromatography: 5 to $10 \%$ EtOAc / petroleum ether. ${ }^{1} \mathbf{H}$ NMR $\left(400 \mathrm{MHz}, \mathrm{CDCl}_{3}\right) \delta: 0.16(3 \mathrm{H}, \mathrm{s}), 0.17(3 \mathrm{H}, \mathrm{s}), 0.90(3 \mathrm{H}, \mathrm{t}, \mathrm{J}=$ $7.30 \mathrm{~Hz}), 1.32(2 \mathrm{H}$, sex, J = 7.13 Hz), 1.44-1.50 (2H, m), $1.53(1 \mathrm{H}, \mathrm{t}, \mathrm{J}=4.73 \mathrm{~Hz}), 2.49(1 \mathrm{H}, \mathrm{t}, \mathrm{J}=7.32 \mathrm{~Hz})$, $3.34(1 \mathrm{H}, \mathrm{dd}, \mathrm{J}=5.13 \mathrm{~Hz}, 10.64 \mathrm{~Hz}), 3.47(1 \mathrm{H}, \mathrm{dd}, \mathrm{J}=4.44 \mathrm{~Hz}, 10.67 \mathrm{~Hz}), 7.00(2 \mathrm{H}, \mathrm{d}, \mathrm{J}=6.96 \mathrm{~Hz}), 7.08(1 \mathrm{H}$, $\mathrm{t}, \mathrm{J}=7.38 \mathrm{~Hz}), 7.21(2 \mathrm{H}, \mathrm{t}, \mathrm{J}=7.51 \mathrm{~Hz}) .{ }^{13} \mathrm{C}$ NMR (101 MHz, $\left.\mathrm{CDCl}_{3}\right)$ 8: -2.65, -2.47, 13.95, 22.36, 22.53, 25.85, 27.90, 29.57, 69.62, 109.06, 124.42, 128.32, 128.36, 139.61, 141.36. ${ }^{29}$ Si NMR (80 MHz, CDCl ${ }_{3}$ ) : 10.19. HRMS (APCI): $\mathrm{m} / \mathrm{z}$ calculated for $\mathrm{C}_{17} \mathrm{H}_{25} \mathrm{Si}[\mathrm{M}-(\mathrm{OH})]^{+}: 257.1720$, found 257.1718 . 
Scale, physical description, yield: $11 \mathrm{mmol}$, pale-yellow oil. $80 \%$ yield, $1.8 \mathrm{gr}$. Flash chromatography: 5 to $10 \%$ EtOAc / petroleum ether. ${ }^{1} \mathbf{H}$ NMR $\left(400 \mathrm{MHz}, \mathrm{CDCl}_{3}\right) \delta: 0.42(3 \mathrm{H}, \mathrm{s}), 0.91(3 \mathrm{H}, \mathrm{t}, \mathrm{J}=7.35 \mathrm{~Hz}), 107-$ $1.16(4 \mathrm{H}, \mathrm{m}) 1.37(2 \mathrm{H}$, sex, $\mathrm{J}=7.39 \mathrm{~Hz}), 1.57(2 \mathrm{H}$, quin, $\mathrm{J}=7.45 \mathrm{~Hz}), 1.68(1 \mathrm{H}, \mathrm{t}, \mathrm{J}=4.66 \mathrm{~Hz}), 2.11(2 \mathrm{H}$, quin, $\mathrm{J}=8.25), 2.57(2 \mathrm{H}, \mathrm{t}, \mathrm{J}=7.29 \mathrm{~Hz}), 3.50(2 \mathrm{H}, \mathrm{ddd}, \mathrm{J}=4.73 \mathrm{~Hz}, 10.74 \mathrm{~Hz}, 17.89 \mathrm{~Hz}) .{ }^{13} \mathrm{C} \mathrm{NMR}\left(101 \mathrm{MHz}, \mathrm{CDCl}_{3}\right)$ 8: $-0.90,13.90,14.93,15.20,18.50,22.49,22.72,28.02,29.64,69.42,108.91,142.02 .{ }^{29}$ Si NMR (80 MHz, $\mathrm{CDCl}_{3}$ ) $\delta:$ 1.73. HRMS (APCI): $\mathrm{m} / \mathrm{z}$ calculated for $\mathrm{C}_{12} \mathrm{H}_{21} \mathrm{OSi}[\mathrm{M}-\mathrm{H}]^{+}:$209.1356, found 209.1370.

\section{(2-(dimethyl(phenyl)silyl)-3-(3-((triisopropylsilyl)oxy)propyl)cycloprop-2-en-1-yl)methanol (11e)}

Scale, physical description, yield: $6 \mathrm{mmol}$, pale-yellow oil. $82 \%$ yield, 2.07 gr. Flash chromatography: 5 to $10 \%$ EtOAc / petroleum ether. ${ }^{1} \mathrm{H}$ NMR $\left(400 \mathrm{MHz}, \mathrm{CDCl}_{3}\right) \delta: 0.45(6 \mathrm{H}, \mathrm{s}), 1.05-1.07(21 \mathrm{H}, \mathrm{m}), 1.66(1 \mathrm{H}$, $\mathrm{t}, \mathrm{J}=4.74 \mathrm{~Hz}), 1.80(2 \mathrm{H}, \mathrm{sex}, \mathrm{J}=6.70 \mathrm{~Hz}), 2.69(1 \mathrm{H}, \mathrm{t}, \mathrm{J}=7.33 \mathrm{~Hz}), 2.49(1 \mathrm{H}, \mathrm{t}, \mathrm{J}=7.32 \mathrm{~Hz}), 3.40(1 \mathrm{H}, \mathrm{dd}, \mathrm{J}$ $=5.13 \mathrm{~Hz}, 10.81 \mathrm{~Hz}), 3.52(1 \mathrm{H}, \mathrm{dd}, \mathrm{J}=4.36 \mathrm{~Hz}, 10.77 \mathrm{~Hz}), 3.71(2 \mathrm{H}, \mathrm{t}, \mathrm{J}=6.11 \mathrm{~Hz}), 7.36-7.38(3 \mathrm{H}, \mathrm{m}), 7.55-$ $7.57(2 \mathrm{H}, \mathrm{m}) .{ }^{13} \mathrm{C}$ NMR $\left(101 \mathrm{MHz}, \mathrm{CDCl}_{3}\right)$ 8: $-2.15,-1.93,12.10,18.14,22.54,24.58,30.87,62.50,69.72$, 109.53, 128.12, 129.50, 133.72, 137.71, 141.14. ${ }^{29} \mathrm{Si} \mathrm{NMR} \mathrm{(80} \mathrm{MHz,} \mathrm{CDCl}_{3}$ ) 8: -15.57, 12.70. HRMS (APCI): $\mathrm{m} / \mathrm{z}$ calculated for $\mathrm{C}_{24} \mathrm{H}_{41} \mathrm{OSi}_{2}[\mathrm{M}-(\mathrm{OH})]^{+}: 401.2690$, found 401.2711 . 
Scale, physical description, yield: $3 \mathrm{mmol}$, yellow oil. $67 \%$ yield, $593 \mathrm{mg}$. Flash chromatography: 5 to 10 \% EtOAc / petroleum ether. ${ }^{1} \mathrm{H}$ NMR (400 MHz, $\left.\mathrm{CDCl}_{3}\right) \delta: 0.21(3 \mathrm{H}, \mathrm{d}, \mathrm{J}=2.51 \mathrm{~Hz}), 0.22(3 \mathrm{H}, \mathrm{d}, \mathrm{J}=2.51 \mathrm{~Hz})$, $0.89(3 \mathrm{H}, \mathrm{t}, \mathrm{J}=7.34 \mathrm{~Hz}), 1.33-1.42(2 \mathrm{H}, \mathrm{m}), 1.53-1.61(2 \mathrm{H}, \mathrm{m}), 2.51-2.56(2 \mathrm{H}, \mathrm{m}), 3.99(1 \mathrm{H}, \mathrm{d}, \mathrm{J}=10.98 \mathrm{~Hz})$, $4.09(1 \mathrm{H}, \mathrm{d}, \mathrm{J}=10.98 \mathrm{~Hz}), 4.40\left(1 \mathrm{H}\right.$, sep, J = 3.76 Hz), 7.08-7.15 (3H, m), 7.22-7.26 (2H, m). ${ }^{13} \mathrm{C}$ NMR (101 $\mathrm{MHz}, \mathrm{CDCl}_{3}$ ) $\delta:-3.38,-3.27,13.93,22.60,26.15,29.77,33.34,68.55,108.78,125.01,125.99,128.20$, 139.67, 146.47. ${ }^{29} \mathrm{Si} \mathrm{NMR}\left(80 \mathrm{MHz} \mathrm{CDCl}_{3}\right)$ 8: -29.83 . HRMS (APCI): $\mathrm{m} / \mathrm{z}$ calculated for $\mathrm{C}_{16} \mathrm{H}_{23} \mathrm{OSi}[\mathrm{M}-\mathrm{H}]^{+}$: 259.15.13, found 259.1520.

HPLC: The racemic and chiral examples were analyzed using a CHIRALCEL ${ }^{\circledR} A Z-H$ column $(0.46 \mathrm{~cm} \emptyset \times 25 \mathrm{~cm})$, $n$-hexane/isopropanol 95/5, 0.75mL/min, $250 \mathrm{~nm}$. $t_{R}$ (major) $=6.15 \mathrm{~min}, \mathrm{t}_{\mathrm{R}}($ minor $)=6.53 \mathrm{~min}$.
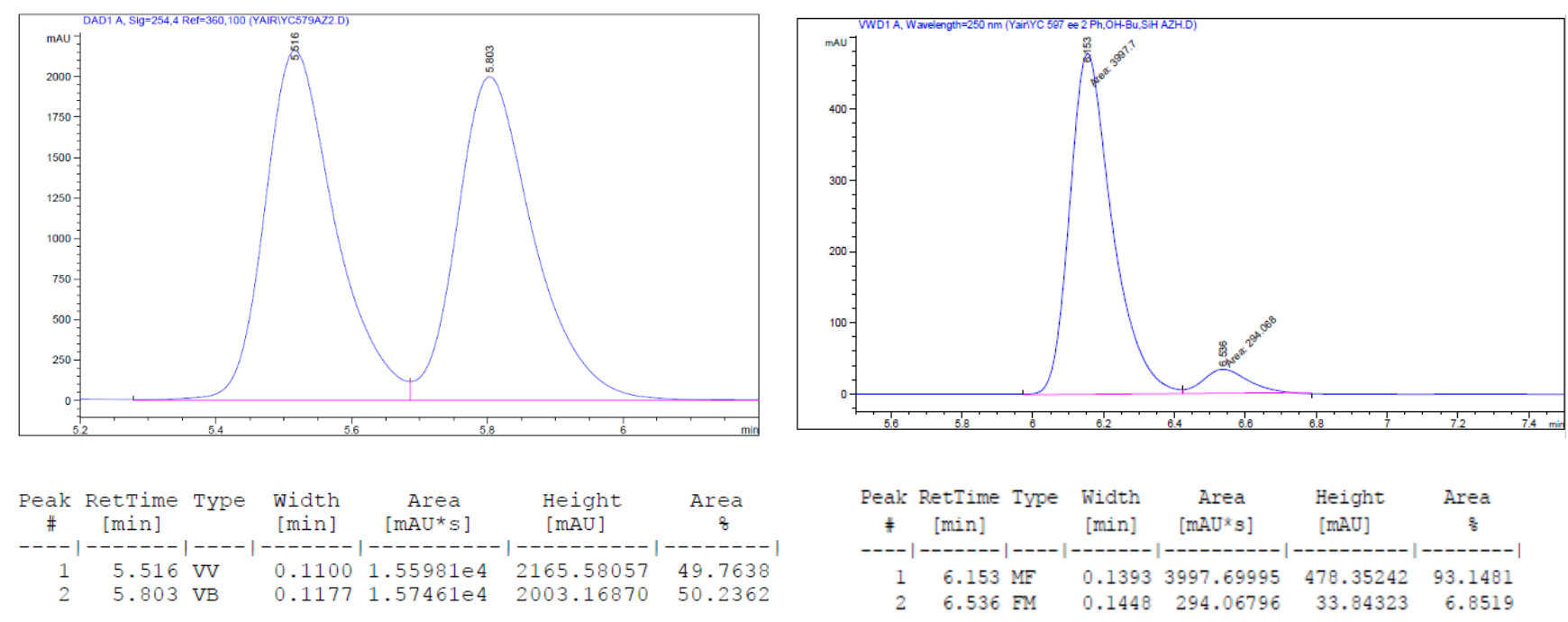

(2-(dimethylsilyl)-3-hexyl-1-phenylcycloprop-2-en-1-yl)methanol (11g)

Scale, physical description, yield: $3 \mathrm{mmol}$, yellow oil. $53 \%$ yield, $504 \mathrm{mg}$. Flash chromatography: 5 to 10 \% EtOAc / petroleum ether. ${ }^{1} \mathrm{H}$ NMR (400 MHz, $\left.\mathrm{CDCl}_{3}\right) \delta: 0.25(3 \mathrm{H}, \mathrm{d}, \mathrm{J}=2.00 \mathrm{~Hz}), 0.26(3 \mathrm{H}, \mathrm{d}, \mathrm{J}=2.00 \mathrm{~Hz})$, $0.89(3 \mathrm{H}, \mathrm{t}, \mathrm{J}=6.91 \mathrm{~Hz}), 1.24-1.33(4 \mathrm{H}, \mathrm{m}), 1.35-1.42(2 \mathrm{H}, \mathrm{m}), 1.58-1.65(2 \mathrm{H}, \mathrm{m}), 2.57(2 \mathrm{H}, \mathrm{td}, \mathrm{J}=7.16 \mathrm{~Hz}$, $5.10 \mathrm{~Hz}), 4.03(1 \mathrm{H}, \mathrm{d}, \mathrm{J}=10.97 \mathrm{~Hz}), 4.13(1 \mathrm{H}, \mathrm{d}, \mathrm{J}=10.97 \mathrm{~Hz}), 4.44(1 \mathrm{H}, \operatorname{sep}, \mathrm{J}=3.81 \mathrm{~Hz}), 7.12-7.19(3 \mathrm{H}, \mathrm{m})$, 7.26-7.30 (2H, m). ${ }^{13} \mathrm{C}$ NMR (101 MHz, $\left.\mathrm{CDCl}_{3}\right)$ 8: -3.36, -3.26, 14.19, 22.71, 26.44, 27.66, 29.17, 31.71, 33.35, 68.55, 108.76, 125.01, 125.99, 128.19, 139.70, 146.47. ${ }^{29}$ Si NMR (80 MHz, $\mathrm{CDCl}_{3}$ ) 8: -29.82. HRMS (APCI): $\mathrm{m} / \mathrm{z}$ calculated for $\mathrm{C}_{18} \mathrm{H}_{29} \mathrm{OSi}[\mathrm{M}+\mathrm{H}]^{+}:$289.1982, found 289.1969. 
(2-(dimethylsilyl)-3-hexyl-1-methylcycloprop-2-en-1-yl)methanol (11h)

Scale, physical description, yield: $2.5 \mathrm{mmol}$, Pale-yellow oil. $51 \%$ yield, $280 \mathrm{mg}$. Flash chromatography: 5 to $10 \%$ EtOAc / petroleum ether. ${ }^{1} \mathbf{H}$ NMR $\left(400 \mathrm{MHz} \mathrm{CDCl}_{3}\right) \delta: 0.23(3 \mathrm{H}, \mathrm{d}, \mathrm{J}=1.86 \mathrm{~Hz}), 0.24(3 \mathrm{H}, \mathrm{d}, \mathrm{J}=$ $2.00 \mathrm{~Hz}), 0.89(3 \mathrm{H}, \mathrm{t}, \mathrm{J}=6.90 \mathrm{~Hz}), 1.17(3 \mathrm{H}, \mathrm{s}), 1.26-1.34(4 \mathrm{H}, \mathrm{m}), 1.35-1.39(2 \mathrm{H}, \mathrm{m}), 1.51-1.59(2 \mathrm{H}, \mathrm{m}), 2.51$ $(2 \mathrm{H}, \mathrm{t}, \mathrm{J}=7.39 \mathrm{~Hz}), 3.52(2 \mathrm{H}, \mathrm{s}), 4.35(1 \mathrm{H}, \operatorname{sep}, \mathrm{J}=3.70 \mathrm{~Hz}) .{ }^{13} \mathrm{C} \mathrm{NMR}\left(101 \mathrm{MHz}, \mathrm{CDCl}_{3}\right)$ 8: -3.28, -3.23, 14.22, 22.19, 22.74, 26.39, 27.44, 27.70, 29.20, 31.78, 69.96, 113.95, 148.26. $\left.{ }^{29} \mathrm{Si} \mathrm{NMR} \mathrm{(80} \mathrm{MHz,} \mathrm{CDCl}\right)$ 8: -22.01. HRMS (APCI): $\mathrm{m} / \mathrm{z}$ calculated for $\mathrm{C}_{13} \mathrm{H}_{25} \mathrm{Si}[\mathrm{M}-(\mathrm{OH})]^{+}:$209.1720, found 209.1720. 
5. General procedure for the silylation of cyclopropenes ${ }^{1}$

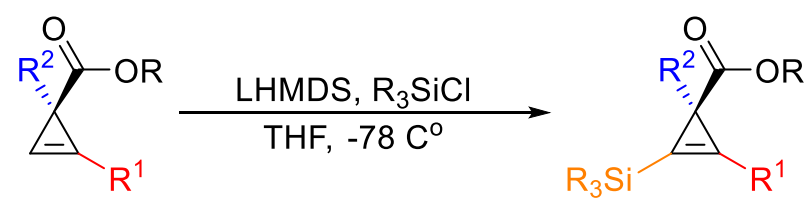

In a flame dried three necked flask $n$-BuLi (1.3 eq.) was added dropwise to a solution of hexamethyldisilazane (1.4 eq.) in THF ( $1 \mathrm{M}$ for cyclopropene) at $-30^{\circ} \mathrm{C}$. After the addition the reaction mixture was stirred for $0.5 \mathrm{hr}$ in $0^{\circ} \mathrm{C}$. In a separate flame dried three necked flask, silyl chloride (1.5 eq.) and cyclopropene (1 eq.) were dissolved in THF (1M for cyclopropene) and cooled to $-78^{\circ} \mathrm{C}$. The freshly prepared LHMDS solution was transferred to the flask containing the cyclopropene via cannula. Upon consumption of cyclopropene (followed by TLC, 10\% EtOAc/ petroleum ether, $p$-anisaldehyde $/ \mathrm{KMnO}_{4}$ ) reaction mixture was warmed to room temperature and quenched with saturated aqueous $\mathrm{NH}_{4} \mathrm{Cl}$ solution. The aqueous layer was extracted three times with $\mathrm{Et}_{2} \mathrm{O}$ and the combined organic phases were washed with brine, dried over $\mathrm{Na}_{2} \mathrm{SO}_{4}$, filtered, and concentrated under reduced pressure. Crude mixture was then purified by flash chromatography using EtOAc / petroleum ether as eluent.

\section{Ethyl 2-butyl-3-(dimethyl(phenyl)silyl)cycloprop-2-enecarboxylate (13a)}

Scale, physical description, yield: $30 \mathrm{mmol}$, pale-yellow oil. $85 \%$ yield. 7.74 gr. Flash chromatography: 3 to $7 \%$ EtOAc / petroleum ether. ${ }^{1} \mathrm{H}$ NMR $\left(400 \mathrm{MHz}^{\mathrm{CDCl}} \mathbf{C D}_{3}\right) \delta: 0.44(3 \mathrm{H}, \mathrm{s}), 0.46(3 \mathrm{H}, \mathrm{s}), 0.87(3 \mathrm{H}, \mathrm{t}, \mathrm{J}=7.33$ $\mathrm{Hz}), 1.19(3 \mathrm{H}, \mathrm{t}, \mathrm{J}=7.13 \mathrm{~Hz}), 1.35(2 \mathrm{H}, \mathrm{sex}, \mathrm{J}=7.41 \mathrm{~Hz}), 1.54(2 \mathrm{H}$, quin, J = $7.41 \mathrm{~Hz}), 2.06(1 \mathrm{H}, \mathrm{s}), 2.54(2 \mathrm{H}$, $\mathrm{td}, \mathrm{J}=1.66 \mathrm{~Hz}, 7.27 \mathrm{~Hz}), 4.06(2 \mathrm{H}, \mathrm{q}, \mathrm{J}=7.14 \mathrm{~Hz}), 7.36-7.38(3 \mathrm{H}, \mathrm{m}), 7.54-7.56(2 \mathrm{H}, \mathrm{m}) .{ }^{13} \mathrm{C} \mathrm{NMR}(\mathbf{1 0 1} \mathrm{MHz}$, $\mathrm{CDCl}_{3}$ ) $\delta:-2.52,-2.27,13.83,14.49,21.36,22.36,26.34,29.13,59.93,102.29,127.99,128.68,129.57$, 133.86, 136.69, 177.00. HRMS (TOF ES+): $\mathrm{m} / \mathrm{z}$ calculated for $\mathrm{C}_{18} \mathrm{H}_{27} \mathrm{O}_{2} \mathrm{Si}[\mathrm{M}+\mathrm{H}]^{+}: 303.1780$, found 303.1786.

\section{Ethyl 2-(benzyldimethylsilyl)-3-butylcycloprop-2-enecarboxylate (13c)}

Scale, physical description, yield: $5 \mathrm{mmol}$, pale-yellow oil. $89 \%$ yield, 1.41 gr. Flash chromatography: 3 to $7 \%$ EtOAc / petroleum ether. ${ }^{1} \mathrm{H}$ NMR $\left(400 \mathrm{MHz}, \mathrm{CDCl}_{3}\right) \delta: 0.14(3 \mathrm{H}, \mathrm{s}), 0.17(3 \mathrm{H}, \mathrm{s}), 0.89(3 \mathrm{H}, \mathrm{t}, \mathrm{J}=7.30$ $\mathrm{Hz}), 1.23(3 \mathrm{H}, \mathrm{t}, \mathrm{J}=7.13 \mathrm{~Hz}), 1.32(2 \mathrm{H}, \operatorname{sex}, \mathrm{J}=7.41 \mathrm{~Hz}), 1.50(2 \mathrm{H}$, quin, J = 7.47 Hz), $1.98(1 \mathrm{H}, \mathrm{s}), 1.24(2 \mathrm{H}$, s), $2.47(2 \mathrm{H}, \mathrm{t}, \mathrm{J}=7.41 \mathrm{~Hz}), 4.10(2 \mathrm{H}, \mathrm{q}, \mathrm{J}=7.14 \mathrm{~Hz}), 7.02(2 \mathrm{H}, \mathrm{d}, \mathrm{J}=7.33 \mathrm{~Hz}), 7.08(1 \mathrm{H}, \mathrm{t}, \mathrm{J}=7.35 \mathrm{~Hz}), 7.21$ $(2 \mathrm{H}, \mathrm{t}, \mathrm{J}=7.55 \mathrm{~Hz}) .{ }^{13} \mathrm{C}$ NMR $\left(101 \mathrm{MHz}, \mathrm{CDCl}_{3}\right) \delta-3.24,-3.15,13.85,14.54,21.14,22.34,25.20,26.28$, 29.03, 59.94, 101.95, 124.44, 128.34, 128.35, 139.08, 177.10. HRMS (TOF ES+): m/z calculated for $\mathrm{C}_{19} \mathrm{H}_{29} \mathrm{O}{ }_{2} \mathrm{Si}[\mathrm{M}+\mathrm{H}]^{+}:$317.1937, found 317.1945. 
Ethyl 2-butyl-3-(1-methylsiletan-1-yl)cycloprop-2-enecarboxylate (13d)

Scale, physical description, yield: $15 \mathrm{mmol}$, pale-yellow oil. $80 \%$ yield, 3.05 gr. Flash chromatography: 3 to $7 \%$ EtOAc / petroleum ether. ${ }^{1} \mathbf{H}$ NMR $\left(400 \mathbf{~ M H z} \mathrm{CDCl}_{3}\right) \delta: 0.45(3 \mathrm{H}, \mathrm{s}), 0.92(3 \mathrm{H}, \mathrm{t}, \mathrm{J}=7.35 \mathrm{~Hz}), 1.03-$ $1.19(4 \mathrm{H}, \mathrm{m}), 1.23(3 \mathrm{H}, \mathrm{t}, \mathrm{J}=7.10 \mathrm{~Hz}), 1.40(2 \mathrm{H}, \operatorname{sex}, \mathrm{J}=7.42 \mathrm{~Hz}), 1.58-1.65(2 \mathrm{H}, \mathrm{m}), 2.13-2.15(2 \mathrm{H}, \mathrm{m}), 2.57$ $(2 \mathrm{H}, \mathrm{dt}, \mathrm{J}=2.91 \mathrm{~Hz}, 7.27 \mathrm{~Hz}), 4.11(1 \mathrm{H}, \mathrm{q}, \mathrm{J}=7.10 \mathrm{~Hz}) .{ }^{13} \mathrm{C}$ NMR $\left(101 \mathrm{MHz}, \mathrm{CDCl}_{3}\right)$ 8: -1.04, 13.89, 14.55, $14.81,16.65,18.61,21.52,22.40,26.47,29.22,60.02,101.92,128.92,176.97$. HRMS (APCI): m/z calculated for $\mathrm{C}_{14} \mathrm{H}_{25} \mathrm{O}_{2} \mathrm{Si}[\mathrm{M}+\mathrm{H}]^{+}: 253.1618$, found 253.1645 .

\section{Ethyl 2-(dimethyl(phenyl)silyl)-3-(3-((triisopropylsilyl)oxy)propyl)cycloprop-2-enecarboxylate (13e)}

Scale, physical description, yield: $8 \mathrm{mmol}$, pale-yellow oil. $77 \%$ yield, 2.84 gr. Flash chromatography: 3 to $7 \%$ EtOAc / petroleum ether. ${ }^{1} \mathrm{H}$ NMR $\left(400 \mathrm{MHz}, \mathrm{CDCl}_{3}\right)$ 8: $0.44(3 \mathrm{H}, \mathrm{s}), 0.46(3 \mathrm{H}, \mathrm{s}), 1.03-1.07(21 \mathrm{H}, \mathrm{m})$, $1.88(3 \mathrm{H}, \mathrm{t}, \mathrm{J}=7.11 \mathrm{~Hz}), 1.78(2 \mathrm{H}$, quin, J = $7.47 \mathrm{~Hz}), 2.07(1 \mathrm{H}, \mathrm{s}), 1.24(2 \mathrm{H}, \mathrm{s}), 2.66(2 \mathrm{H}, \mathrm{t}, \mathrm{J}=7.39 \mathrm{~Hz}), 3.69$ $(2 \mathrm{H}, \mathrm{t}, \mathrm{J}=6.03 \mathrm{~Hz}), 4.06(2 \mathrm{H}, \mathrm{q}, \mathrm{J}=7.11 \mathrm{~Hz}), 7.33-7.37(3 \mathrm{H}, \mathrm{m}), 7.55-56(2 \mathrm{H}, \mathrm{m}) .{ }^{13} \mathrm{C} \mathrm{NMR}\left(101 \mathrm{MHz}, \mathrm{CDCl}_{3}\right)$ ס: $-2.53,-2.26,12.08,14.50,21.40,23.16,30.46,59.95,62.36,120.38,128.01,128.49,129.60,133.86$, 136.62, 176.92. HRMS (APCI): $\mathrm{m} / \mathrm{z}$ calculated for $\mathrm{C}_{26} \mathrm{H}_{45} \mathrm{O}_{3} \mathrm{Si}_{2}[\mathrm{M}+\mathrm{H}]^{+}: 461.2902$, found 461.2927.

(S)-methyl 2-butyl-3-(dimethylsilyl)-1-phenylcycloprop-2-enecarboxylate (13f)

Scale, physical description, yield: $3.9 \mathrm{mmol}$, yellow oil. $68 \%$ yield, $971 \mathrm{mg}$. Flash chromatography: 3 to 7 \% EtOAc / petroleum ether. ${ }^{1} \mathrm{H}$ NMR (400 MHz, $\left.\mathrm{CDCl}_{3}\right) \delta: 0.21(3 \mathrm{H}, \mathrm{d}, \mathrm{J}=3.67 \mathrm{~Hz}), 0.25(3 \mathrm{H}, \mathrm{d}, \mathrm{J}=3.63 \mathrm{~Hz})$, $0.85(3 \mathrm{H}, \mathrm{t}, \mathrm{J}=7.35 \mathrm{~Hz}), 1.31-1.37(2 \mathrm{H}, \mathrm{m}), 1.53-1.60(2 \mathrm{H}, \mathrm{m}), 2.56(2 \mathrm{H}, \mathrm{t}, \mathrm{J}=7.29 \mathrm{~Hz}), 3.60(3 \mathrm{H}, \mathrm{s}), 4.40-$ 4.43 (1H, m), 7.11-7.14 (1H, m), 7.19-7.22 (4H, m). $\left.{ }^{13} \mathrm{C} \mathrm{NMR} \mathrm{(101} \mathrm{MHz,} \mathrm{CDCl}\right)$ 8: -4.05, -3.97, 13.81, 22.38, 25.65, 29.24, 34.53, 51.78, 105.56, 125.92, 127.98, 128.28, 132.69, 142.57, 176.17. HRMS (APCI): m/z calculated for $\mathrm{C}_{17} \mathrm{H}_{25} \mathrm{O}_{2} \mathrm{Si}[\mathrm{M}+\mathrm{H}]^{+}: 289.1618$, found 289.1634 .

\section{Methyl 2-(dimethylsilyl)-3-hexyl-1-phenylcycloprop-2-enecarboxylate (13g)}

Scale, physical description, yield: $3.5 \mathrm{mmol}$, yellow oil. 93\% yield 1.04 gr. Flash chromatography: 3 to 7 \% EtOAc / petroleum ether. ${ }^{1} \mathrm{H}$ NMR (400 MHz, $\left.\mathrm{CDCl}_{3}\right) \delta: 0.26(3 \mathrm{H}, \mathrm{d}, \mathrm{J}=3.76 \mathrm{~Hz}), 0.30(3 \mathrm{H}, \mathrm{d}, \mathrm{J}=3.72 \mathrm{~Hz})$, $0.88(3 \mathrm{H}, \mathrm{t}, \mathrm{J}=6.77 \mathrm{~Hz}), 1.26-1.37(6 \mathrm{H}, \mathrm{m}), 1.57-1.66(2 \mathrm{H}, \mathrm{m}), 2.60(2 \mathrm{H}, \mathrm{t}, \mathrm{J}=7.35 \mathrm{~Hz}), 3.66(3 \mathrm{H}, \mathrm{s}), 4.46$ $\left(1 \mathrm{H}\right.$, sep, J = 3.64), 7.15-7.20 (1H, m), 7.26-7.27 (4H, m). ${ }^{13}$ C NMR (101 MHz, CDCl $)$ 8: -3.99, -3.91, 14.18, 
$22.68,22.59,27.20,29.00,31.64,34.57,51.84,105.55,125.96,128.02,128.32,132.77,142.61,176.24$. HRMS (APCI): $\mathrm{m} / \mathrm{z}$ calculated for $\mathrm{C}_{19} \mathrm{H}_{29} \mathrm{O}_{2} \mathrm{Si}[\mathrm{M}+\mathrm{H}]^{+}:$317.1931, found 317.1924.

\section{Ethyl 2-(dimethylsilyl)-3-hexyl-1-methylcycloprop-2-enecarboxylate (13h)}

Scale, physical description, yield: $13 \mathrm{mmol}$, pale-yellow oil. $18 \%$ yield, $640 \mathrm{mg}$. Flash chromatography: 3 to $7 \%$ EtOAc / petroleum ether. ${ }^{1} \mathrm{H}$ NMR $\left(400 \mathrm{MHz}, \mathrm{CDCl}_{3}\right) \delta: 0.23(6 \mathrm{H}, \mathrm{t}, \mathrm{J}=3.96), 0.89(3 \mathrm{H}, \mathrm{t}, \mathrm{J}=6.88 \mathrm{~Hz})$, $1.18(3 \mathrm{H}, \mathrm{t}, \mathrm{J}=7.11 \mathrm{~Hz}), 1.28-1.38(6 \mathrm{H}, \mathrm{m}), 1.32(3 \mathrm{H}, \mathrm{s}), 1.53-1.60(2 \mathrm{H}, \mathrm{m}), 2.48(2 \mathrm{H}, \mathrm{t}, \mathrm{J}=7.26 \mathrm{~Hz}), 3.98-$ $4.10(2 \mathrm{H}, \mathrm{m}), 4.34\left(1 \mathrm{H}\right.$, sep, J = 3.71 Hz). ${ }^{13} \mathrm{C}$ NMR (101 MHz, CDCl $)$ 8: -3.93, -3.90, 14.20, 14.56, 20.80, 22.72, 25.86, 27.24, 29.02, 29.84, 31.71, 60.07, 107.30, 134.42, 178.12. HRMS (APCI): m/z calculated for $\mathrm{C}_{15} \mathrm{H}_{29} \mathrm{O}_{2} \mathrm{Si}[\mathrm{M}+\mathrm{H}]^{+}:$269.1931, found 269.1945. 
6. Crystal data and structure refinement for compund 2ac (CCDC 1942906)

\begin{tabular}{|c|c|}
\hline Crystal data & $2 \mathrm{ac}$ \\
\hline Empirical formula & $\mathrm{C}_{18} \mathrm{H}_{28} \mathrm{O}_{3} \mathrm{Si}$ \\
\hline Formula weight & 320.49 \\
\hline Temperature (K) & 200.15 \\
\hline Wavelength $(\AA)$ & 0.71073 \\
\hline Crystal system, & monoclinic \\
\hline space group & $\mathrm{P} 2_{1}$ \\
\hline $\mathrm{a}(\AA \AA)$ & $8.354(3)$ \\
\hline$b(\AA)$ & $10.708(4)$ \\
\hline$c(\AA)$ & $10.378(4)$ \\
\hline alpha & 90 \\
\hline beta & $90.384(10)$ \\
\hline gamma & 90 \\
\hline Volume $\left(\AA^{3}\right)$ & $928.3(6)$ \\
\hline Z & 2 \\
\hline Calculated density $\left(\mathrm{g} / \mathrm{m}^{3}\right)$ & 1.147 \\
\hline Absorption coefficient $\left(\mathrm{mm}^{-1}\right)$ & 0.136 \\
\hline$F(000)$ & 348.0 \\
\hline Crystal size $\left(\mathrm{mm}^{3}\right)$ & $0.24 \times 0.15 \times 0.12$ \\
\hline 2Theta range & $3.924-49.642$ \\
\hline Reflection collected/unique & $5755 / 2894$ \\
\hline Rint & 0.1173 \\
\hline Completeness (\%) & 99.1 \\
\hline Absorption correction & semi-empirical \\
\hline Data/restraints/ parameters & $2894 / 1 / 205$ \\
\hline Goodness-of-fit on $\mathrm{F}^{2}$ & 1.031 \\
\hline R1, wR2 [I>2sigma(I)] & $\mathrm{R}_{1}=0.0890, w \mathrm{R}_{2}=0.2054$ \\
\hline R1, wR2 (all data) & $\mathrm{R}_{1}=0.1182, \mathrm{wR}_{2}=0.2198$ \\
\hline Largest diff. peak and hole & $0.46 /-0.43$ \\
\hline Diffractometer & Bruker Apex II \\
\hline
\end{tabular}<smiles>C[Si](O)(c1ccccc1)C1(C(=O)O)C(CO)C1(Br)Br</smiles>

12c

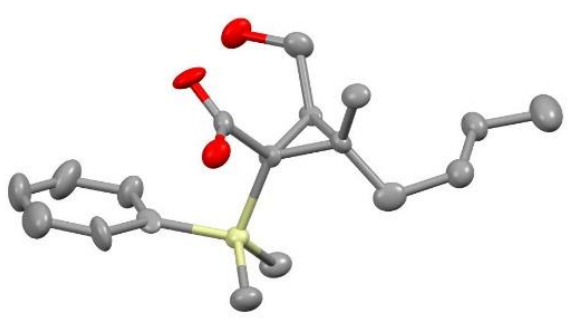

7. Refernces.

1. Li, C.; Zeng, Y.; Zhang, H.; Feng, J.; Zhang, Y.; Wang, J., Gold(I)-Catalyzed Cycloisomerization of Enynes Containing Cyclopropenes. Angew. Chem. Int. Ed. 2010, 49, 6413-6417. 
8. NMR spectra of cyclopropylsilanes $\mathbf{1 2 a - 1 2 z}$
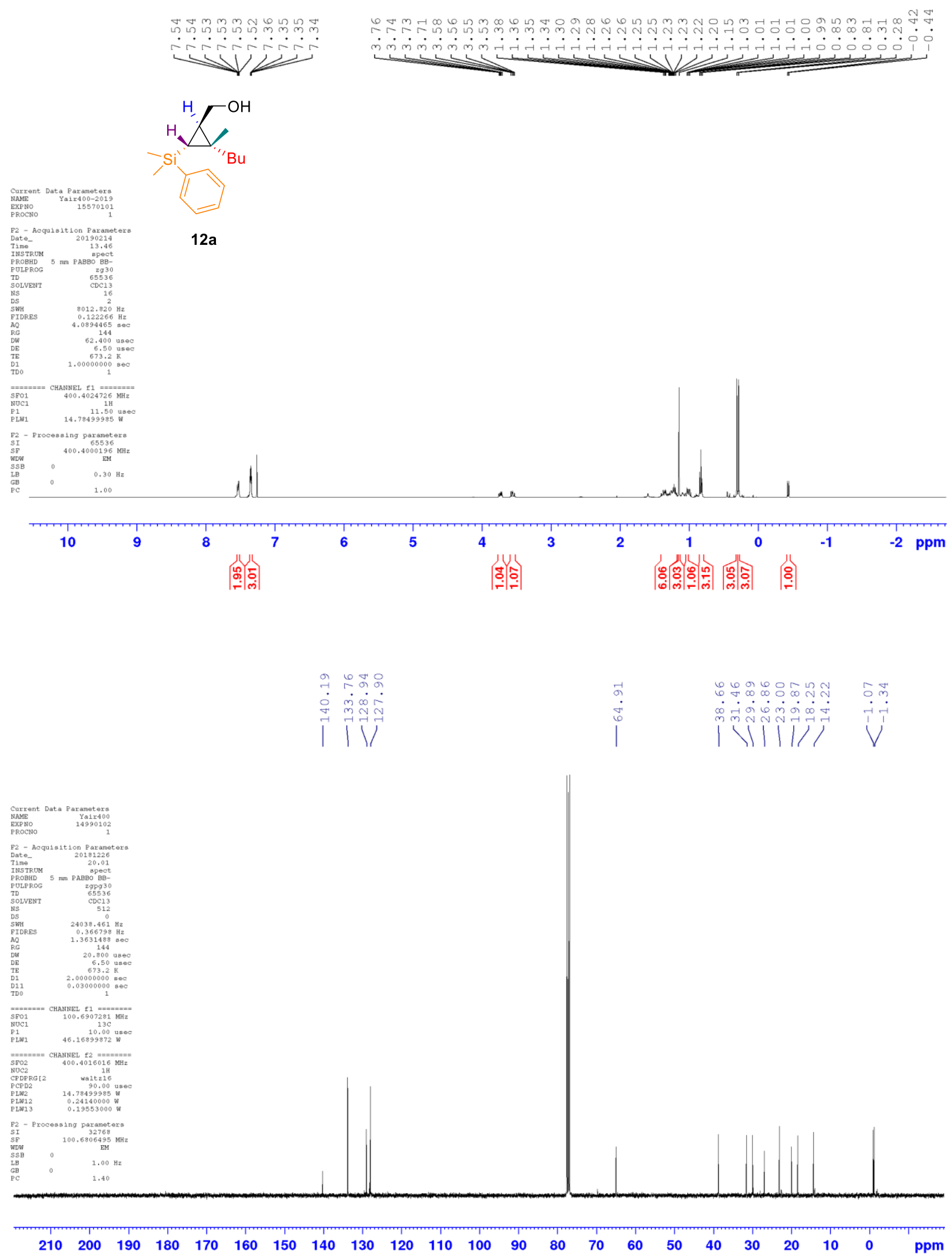


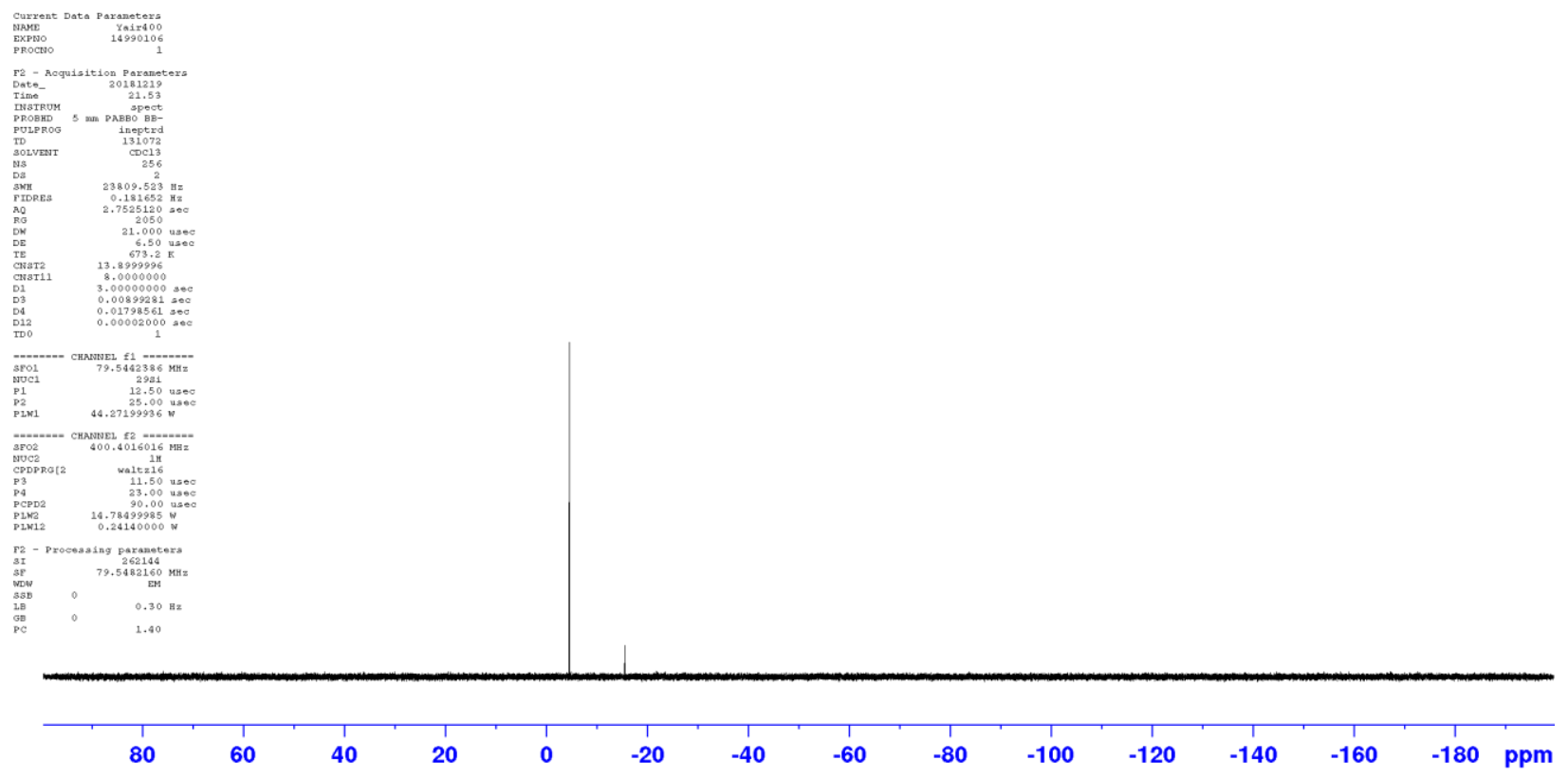



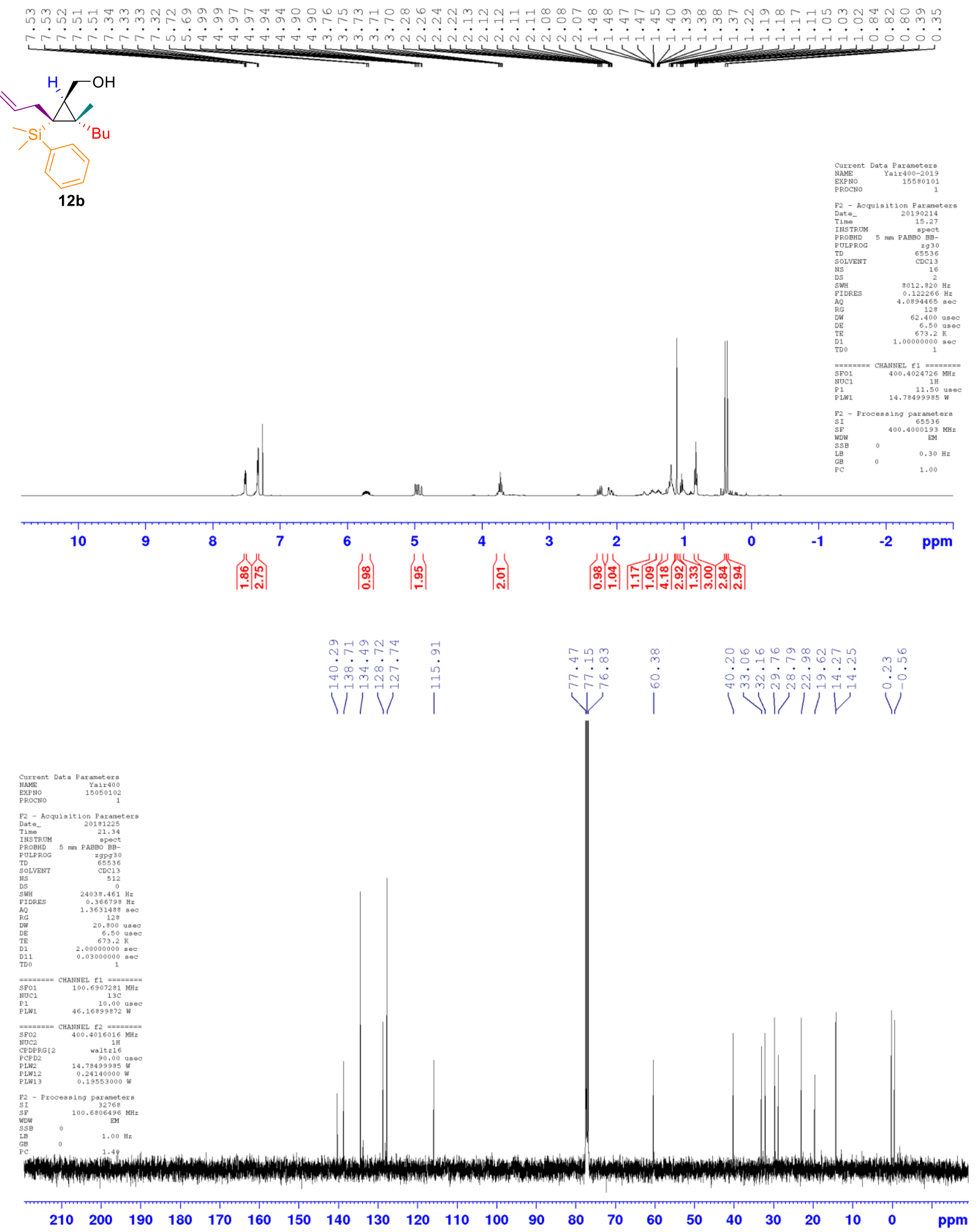


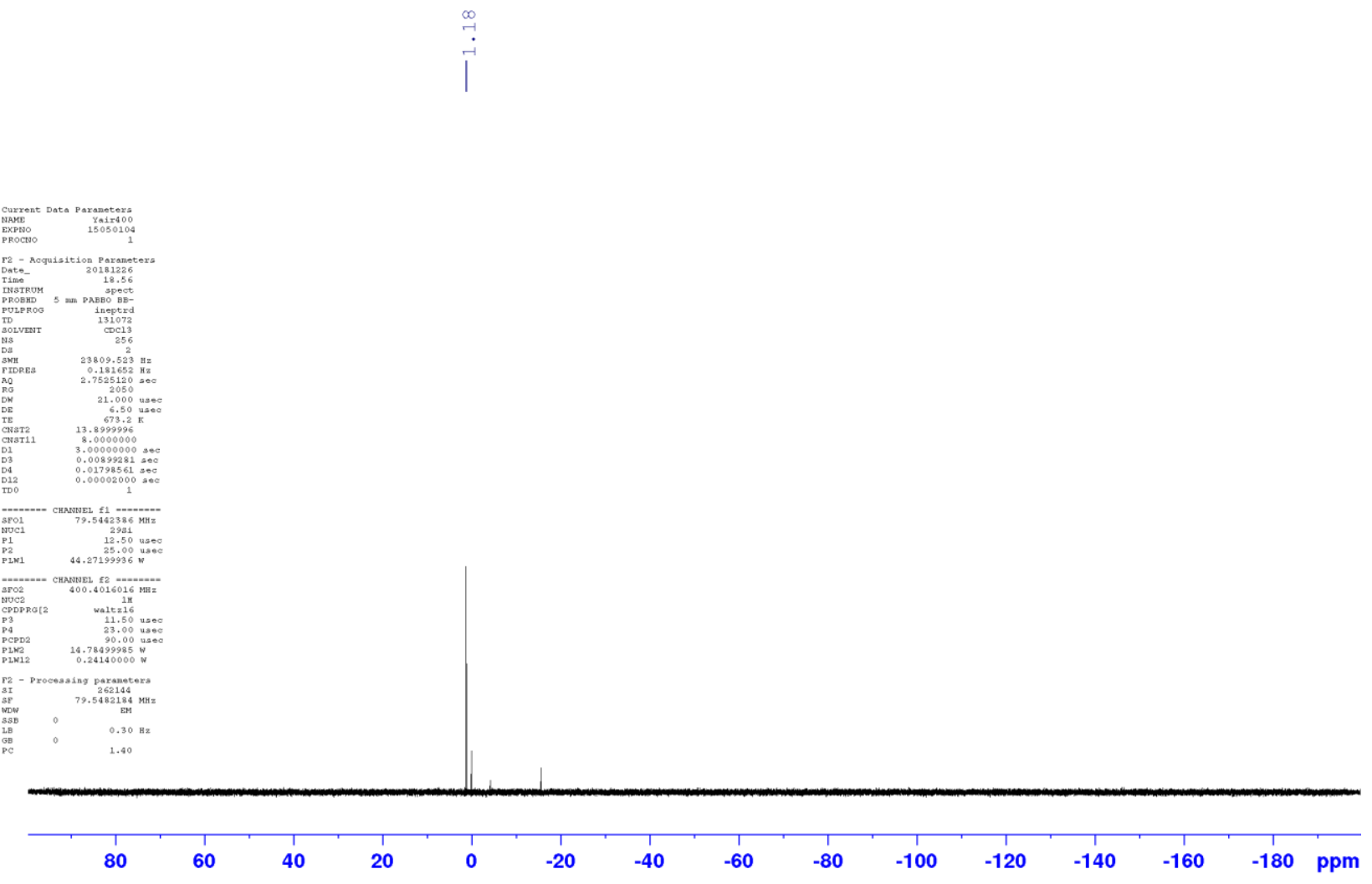



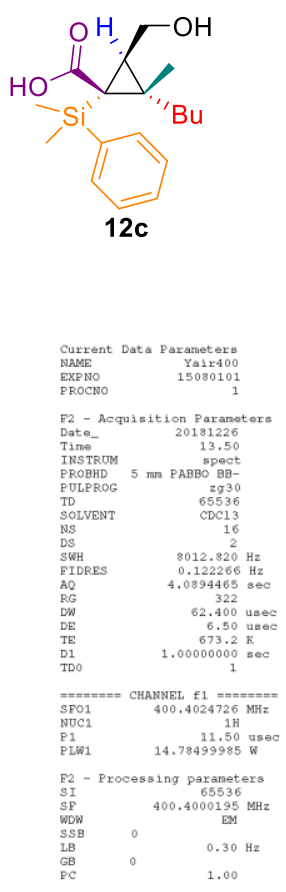

12c

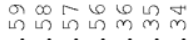

包年

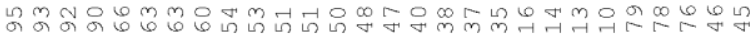

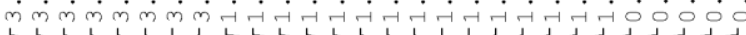

$\rightarrow W$
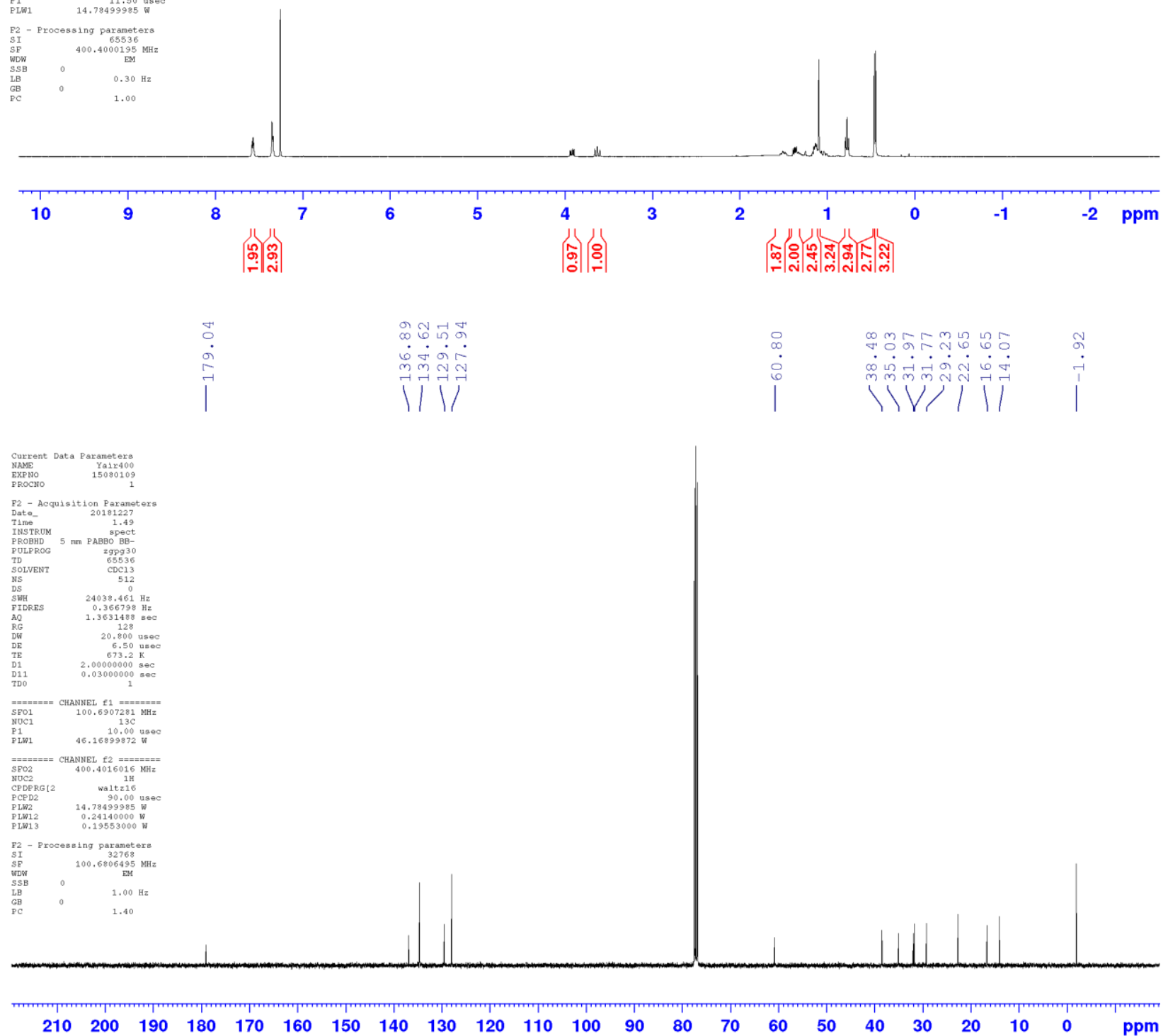


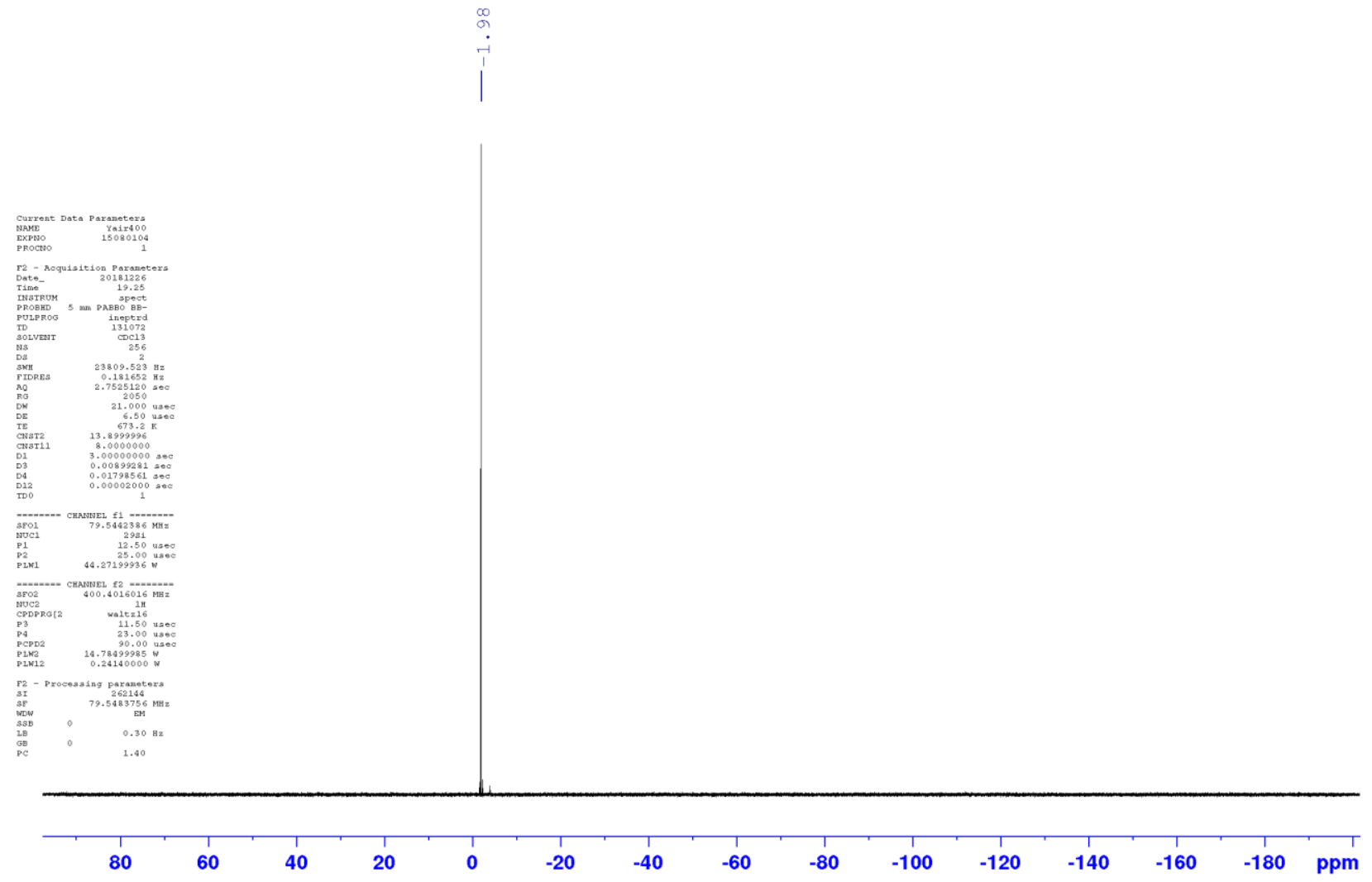



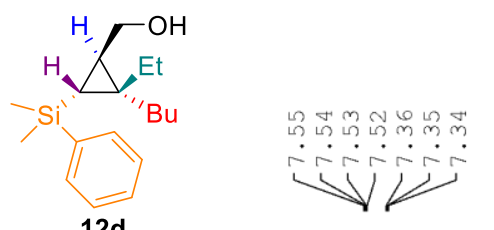

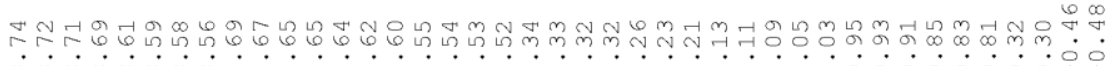
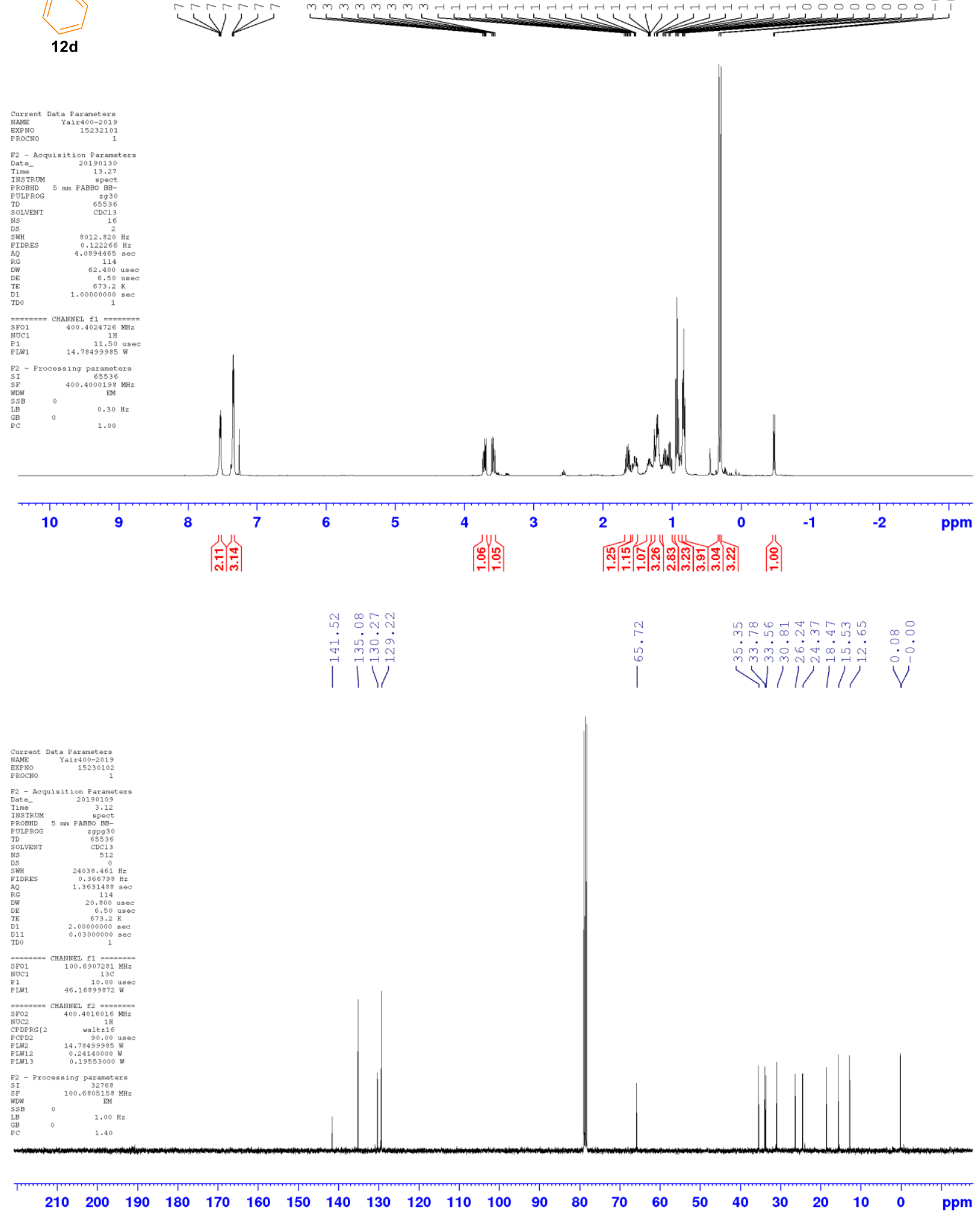


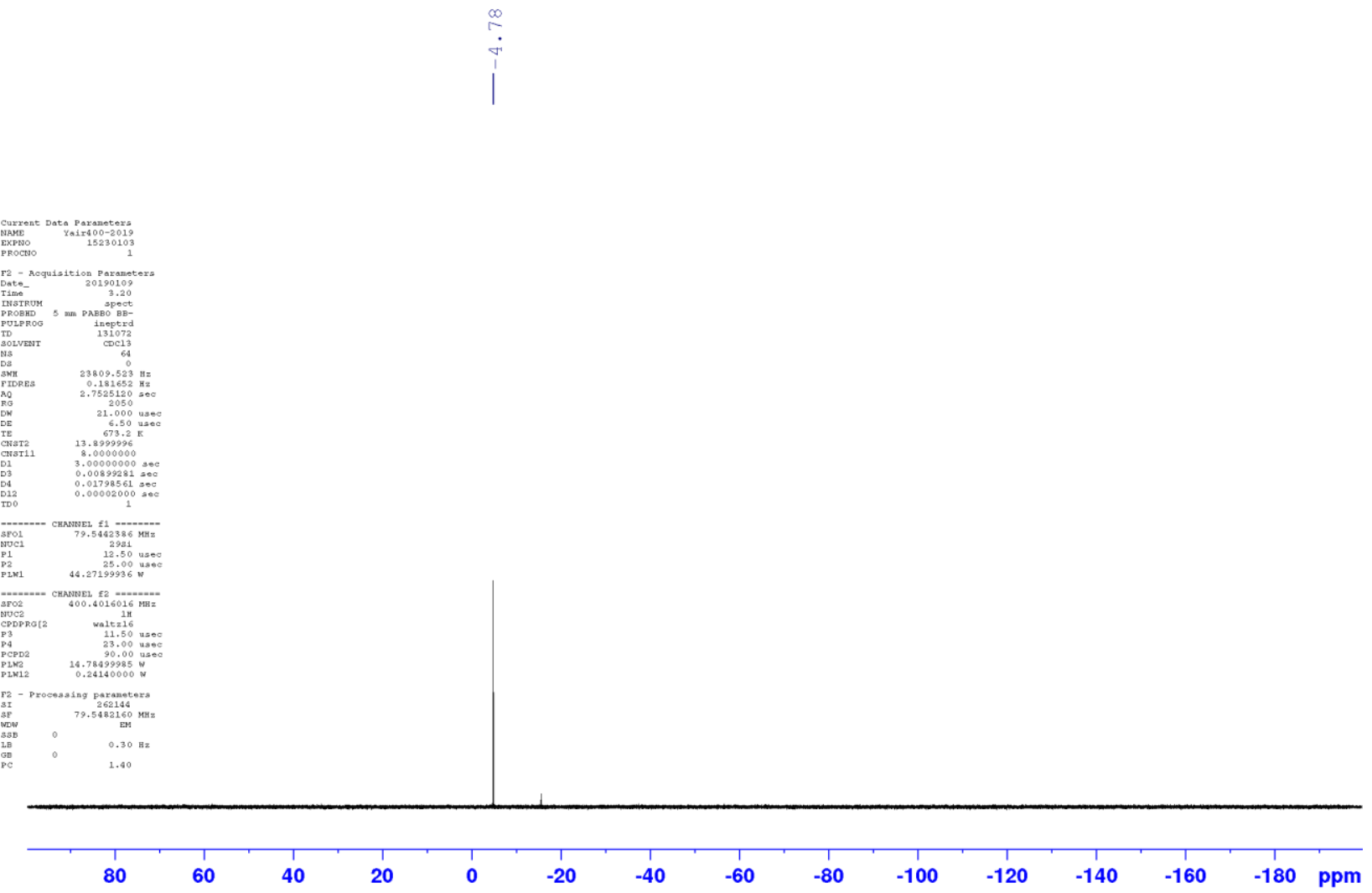




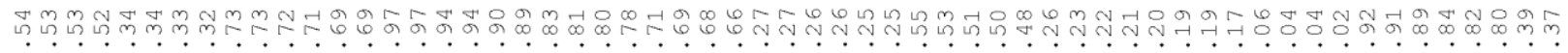
2nank
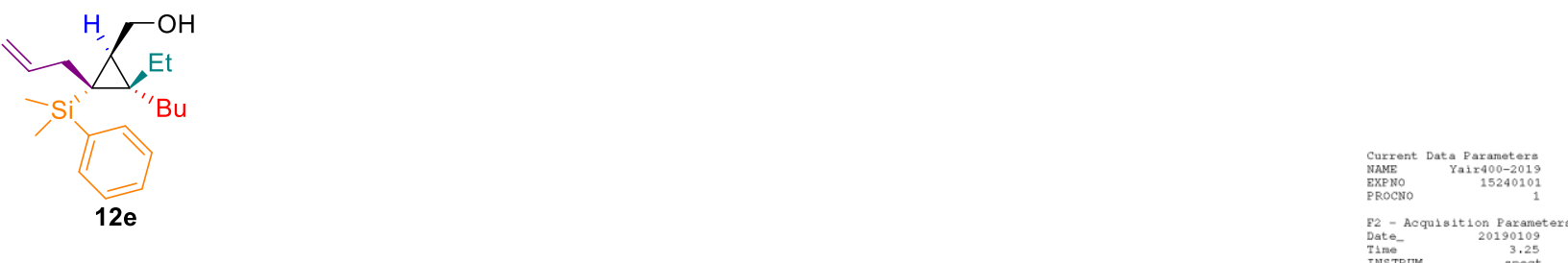

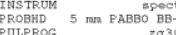
$\begin{array}{rr}65536 \\ \mathrm{COLVENT} & \mathrm{CDC13} \\ \mathrm{NS} & 16\end{array}$

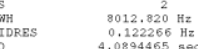
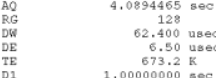

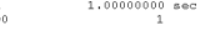

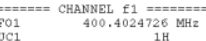

11.50 usec
$14.78499985 \mathrm{~W}$

F2 - Processing parameters

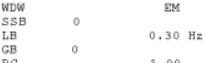

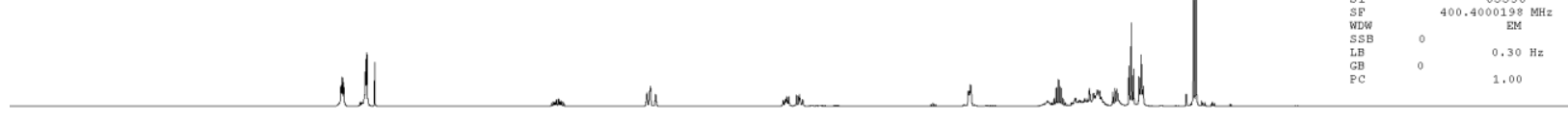

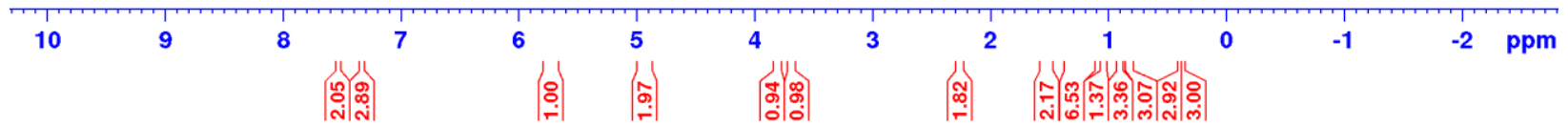
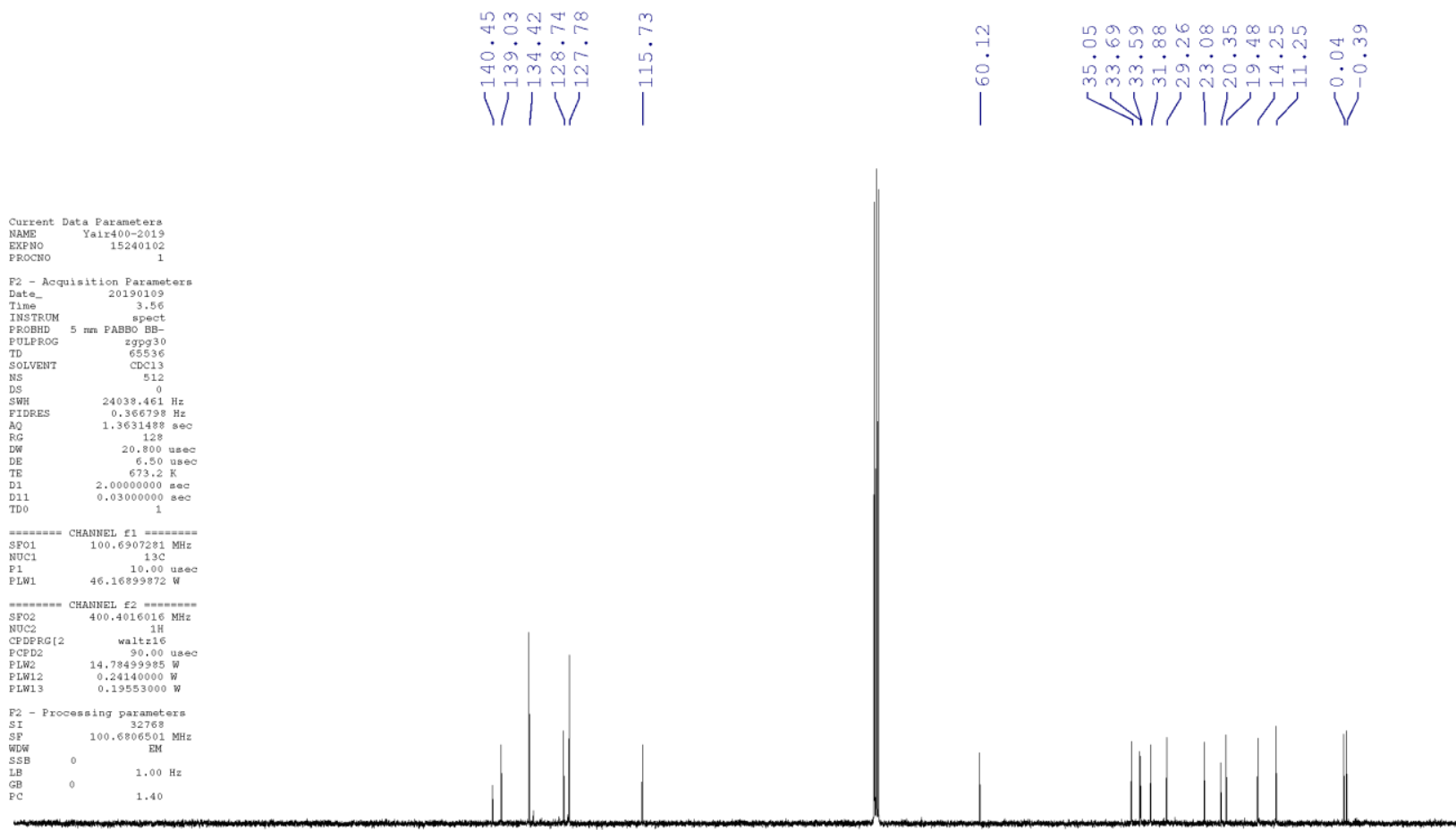

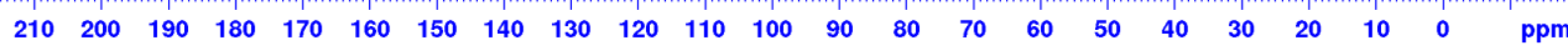




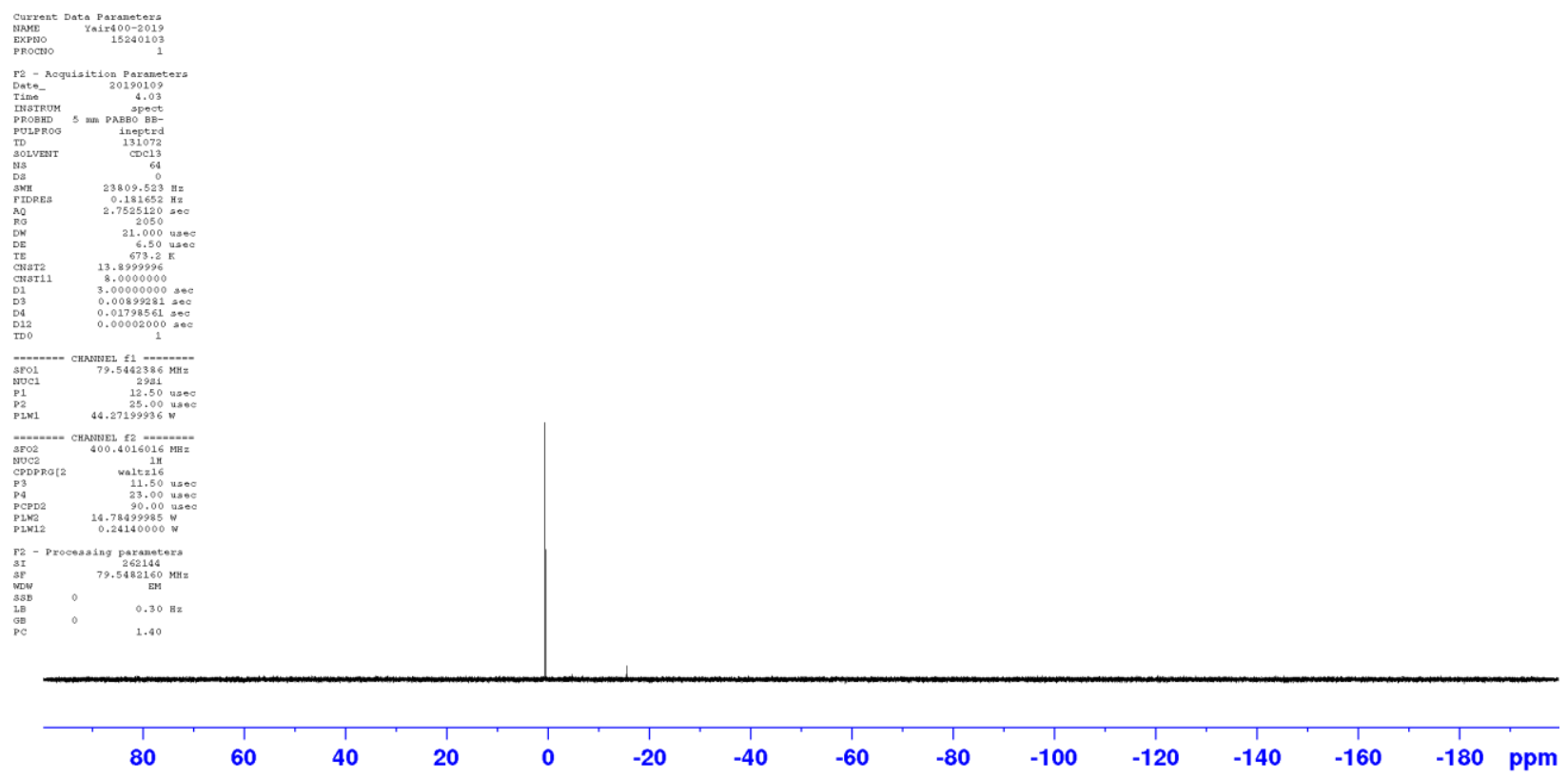




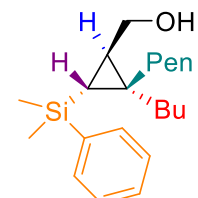

$12 f$

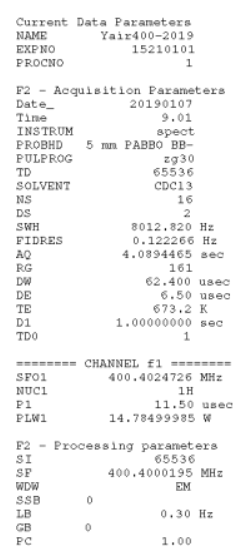

dh All

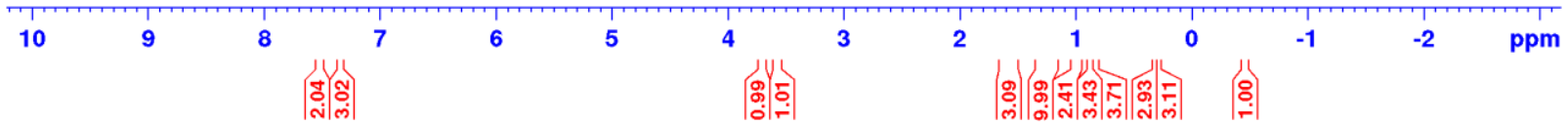
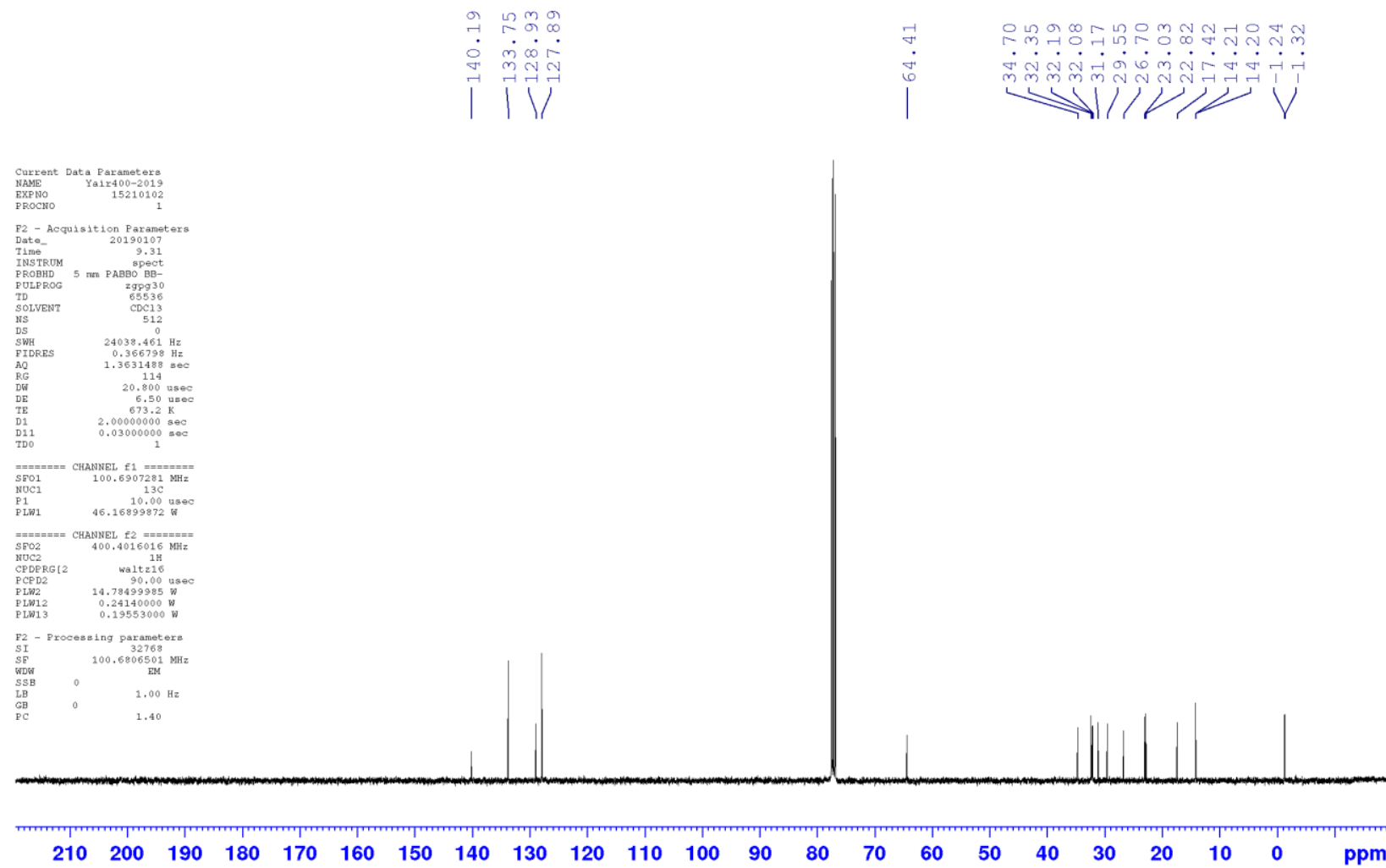


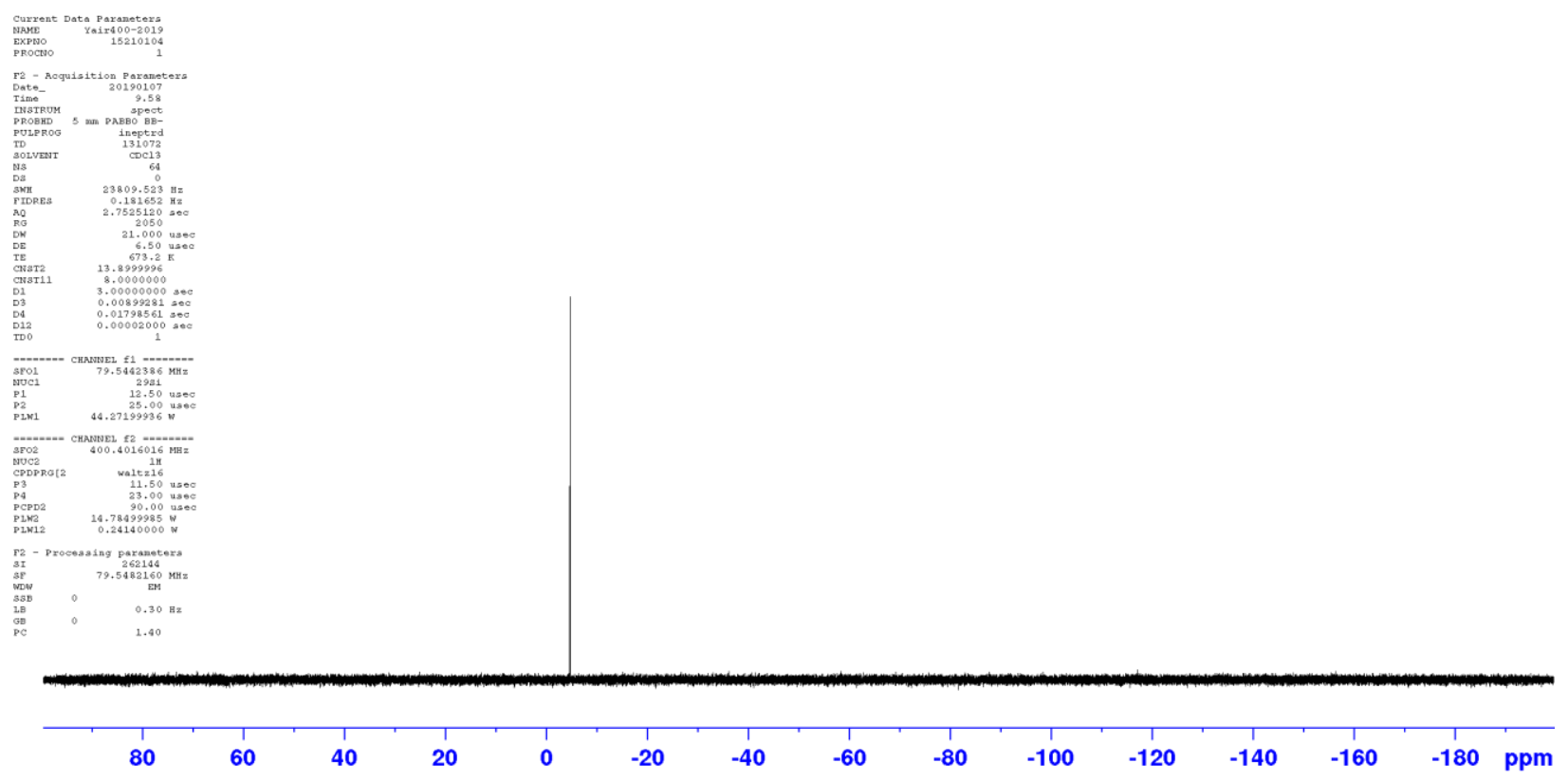




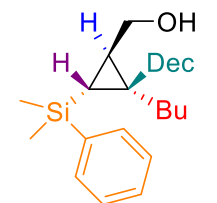

$12 \mathrm{~g}$
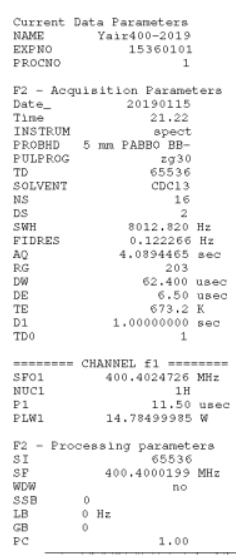

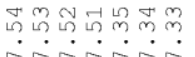

inising

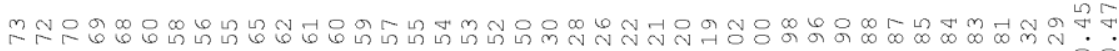

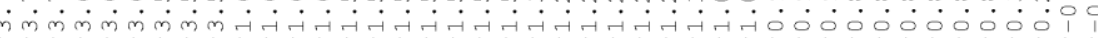

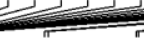
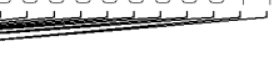
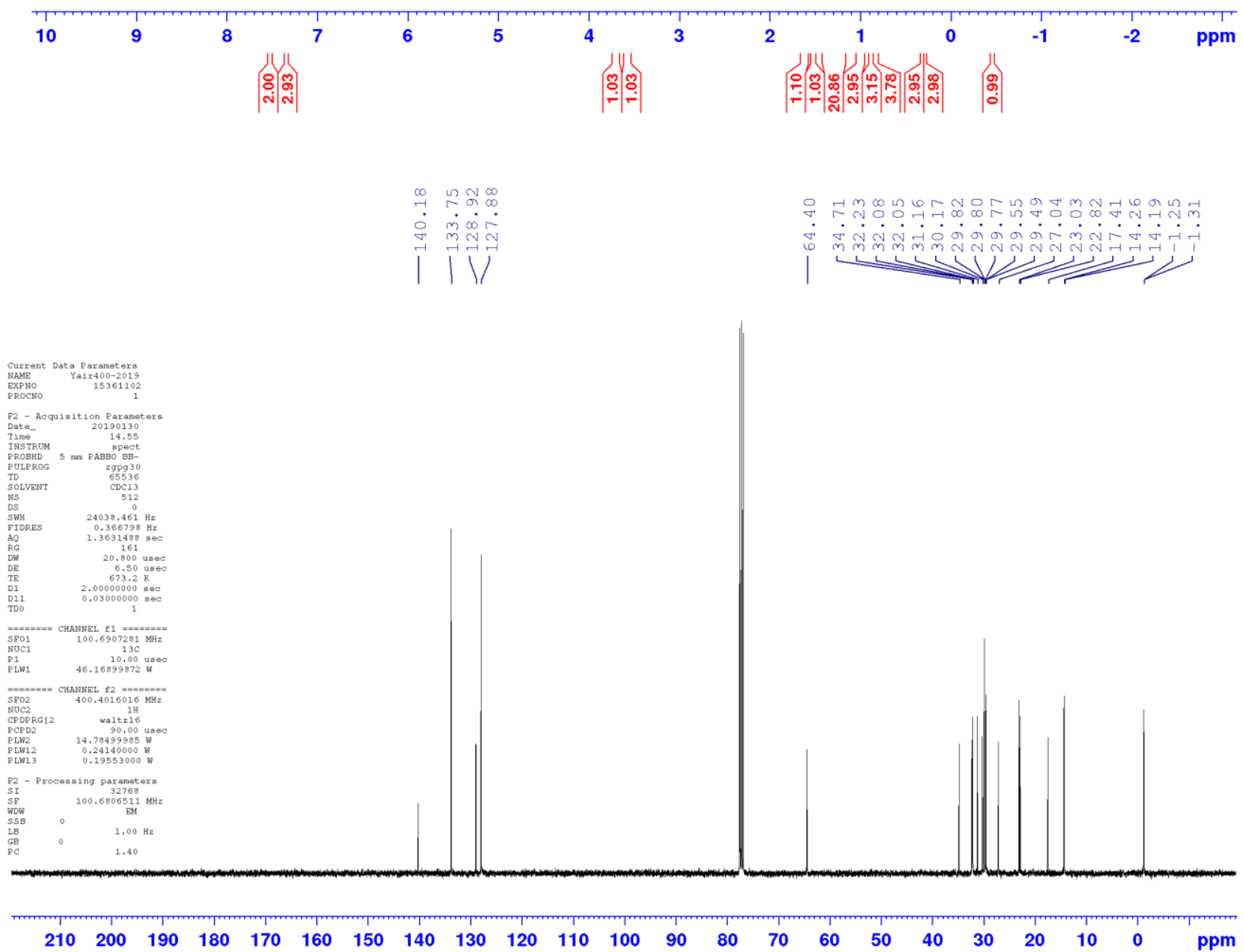


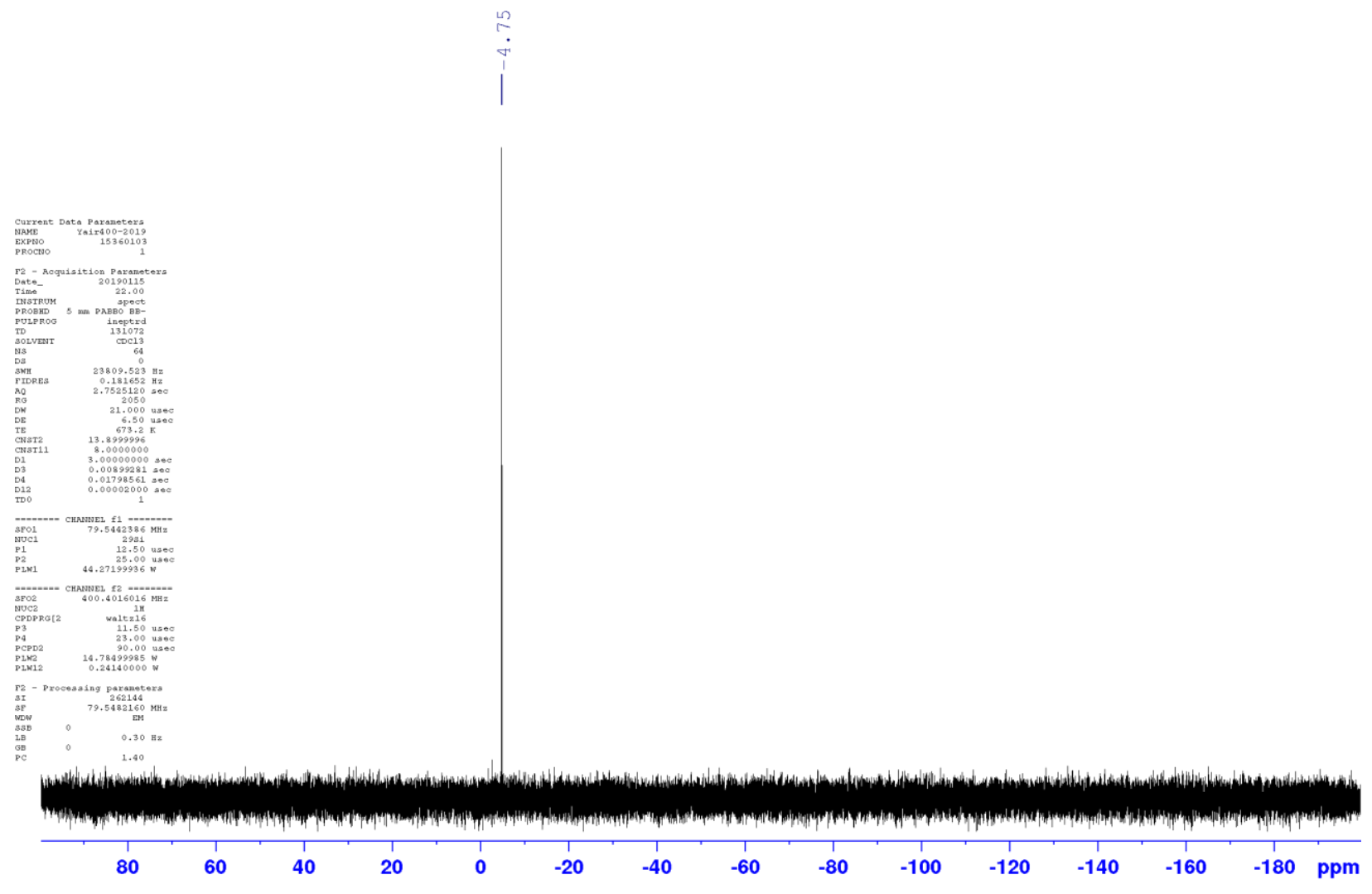




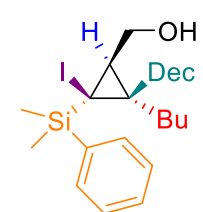

$12 \mathrm{~h}$

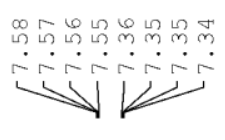

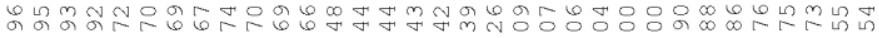

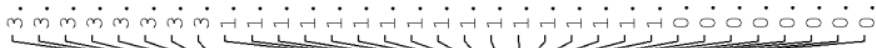

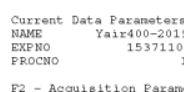

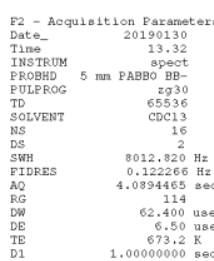

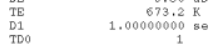

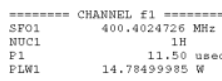

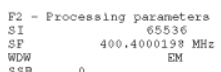

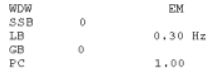

$1 \int$

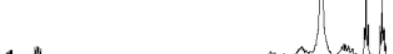

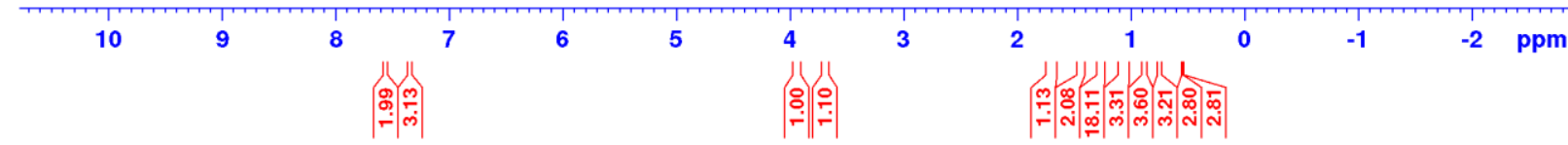
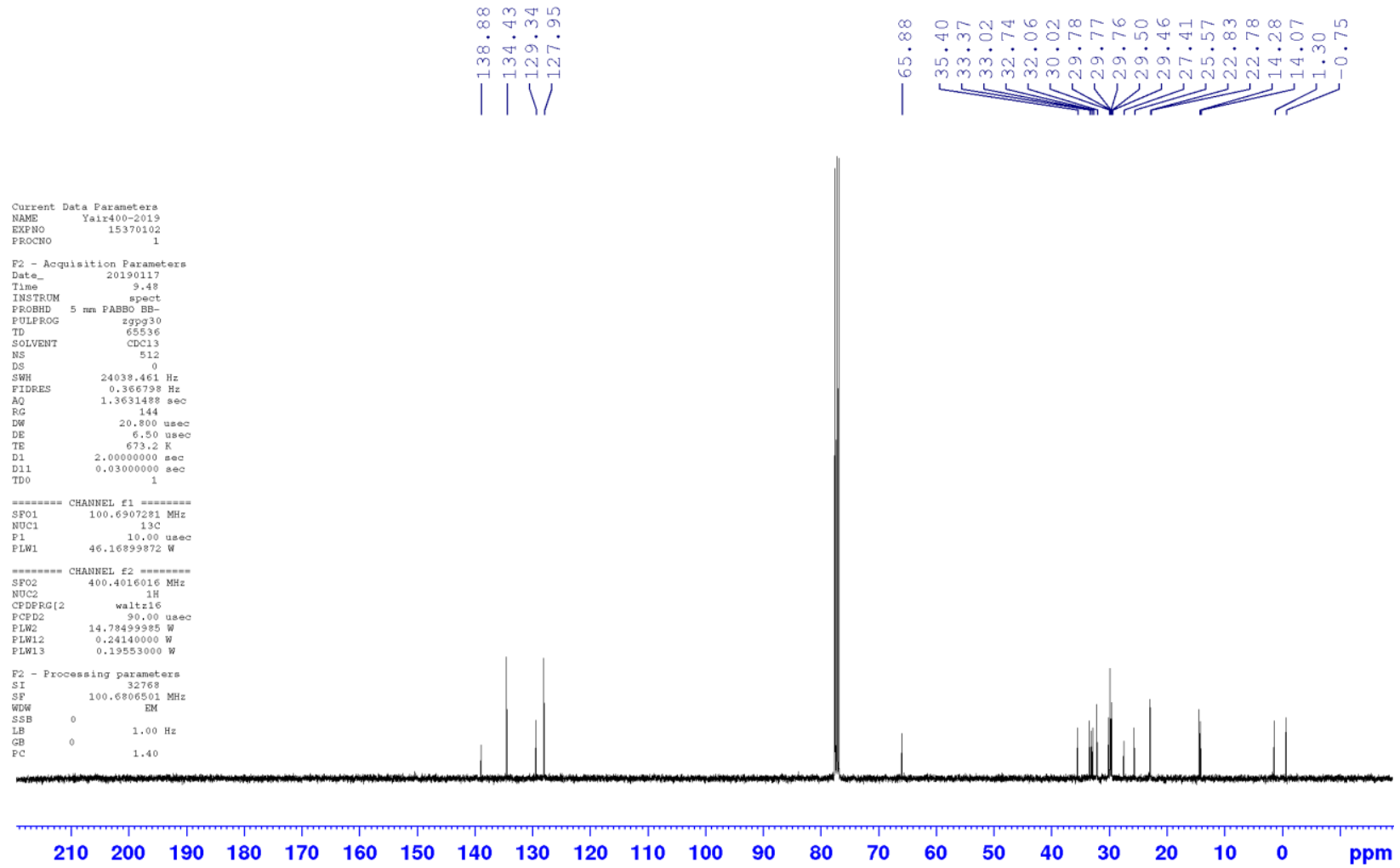


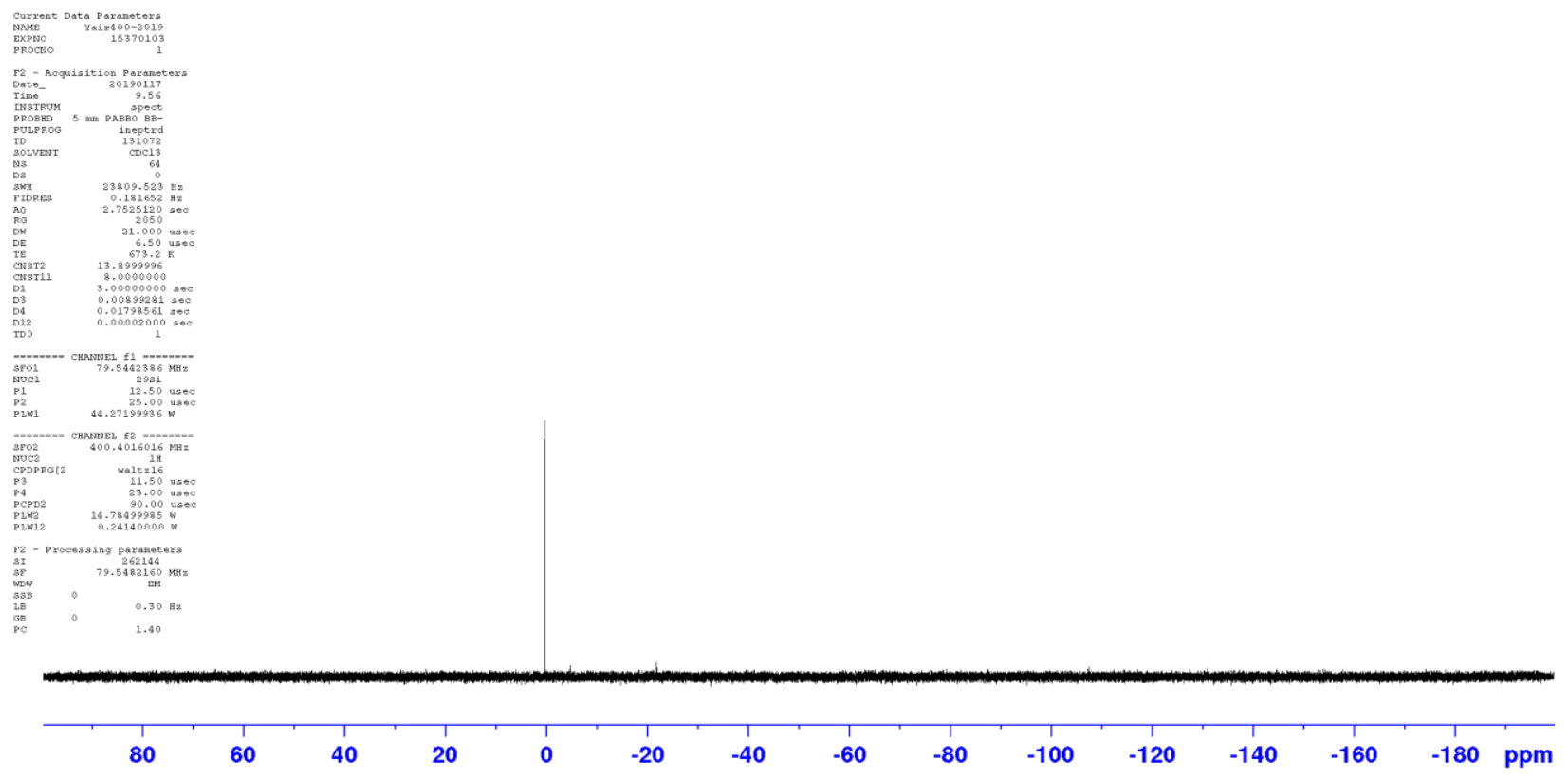



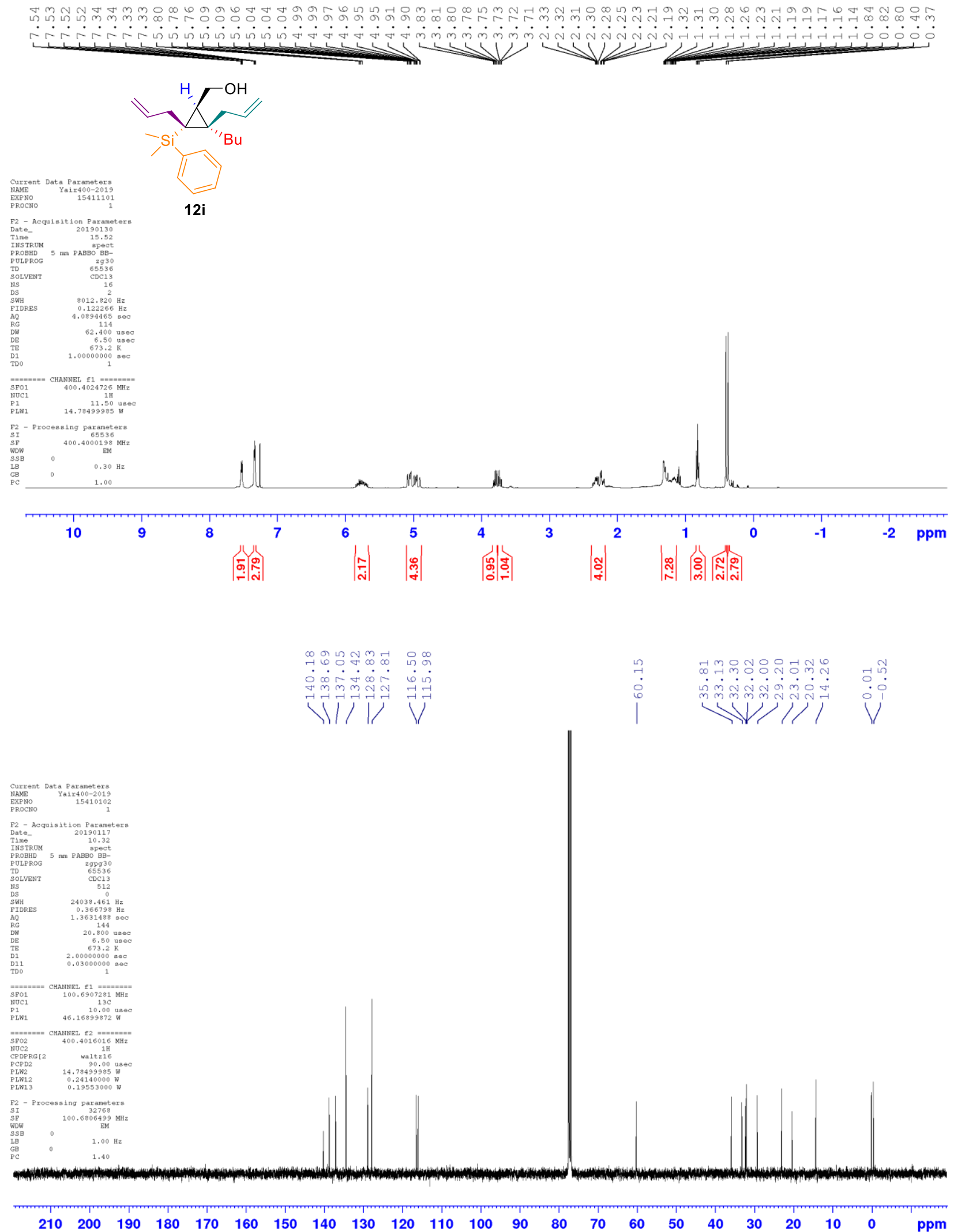


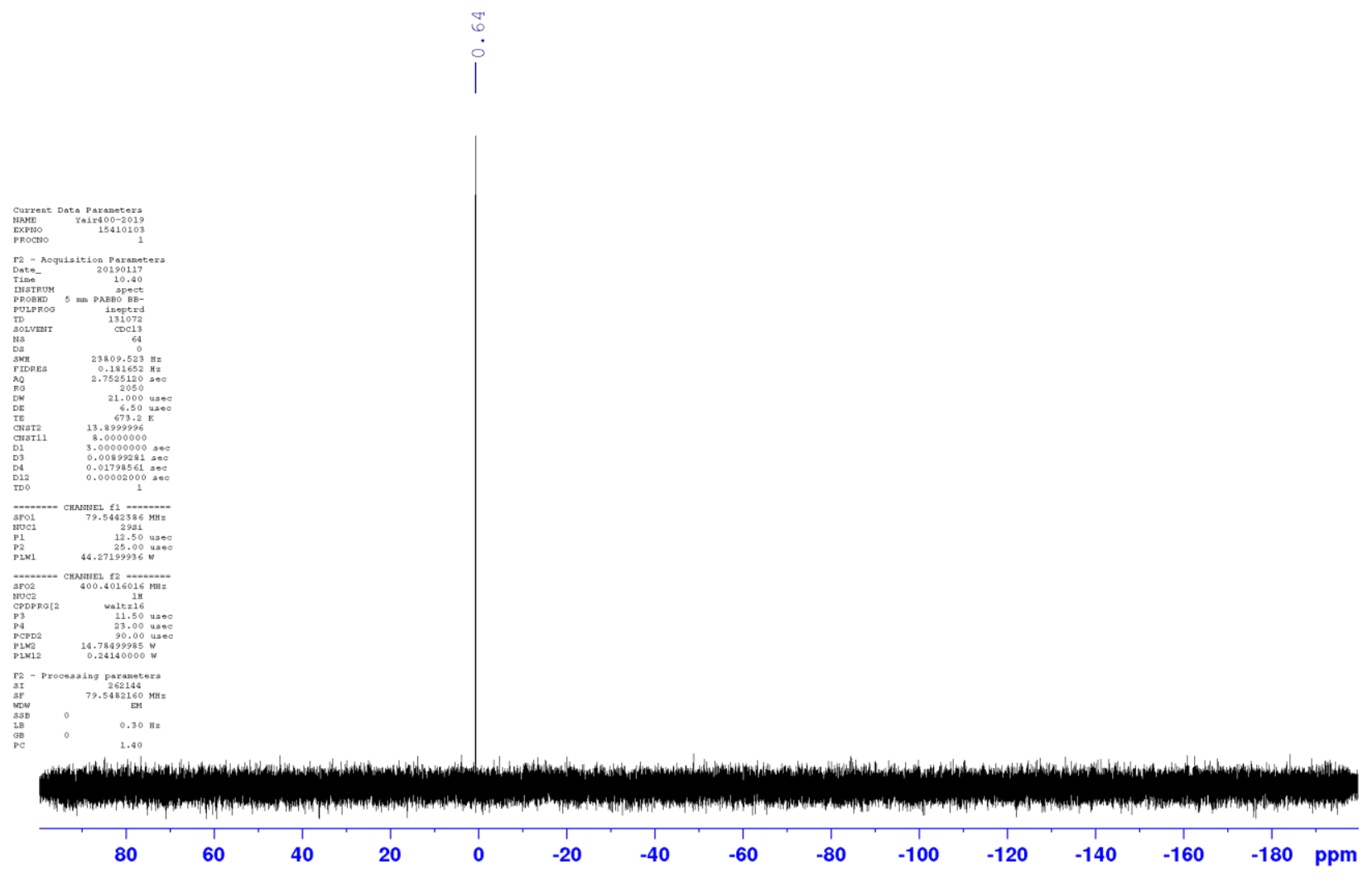




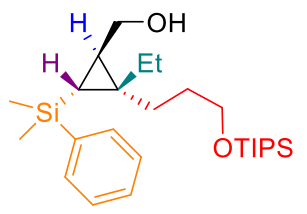

12j
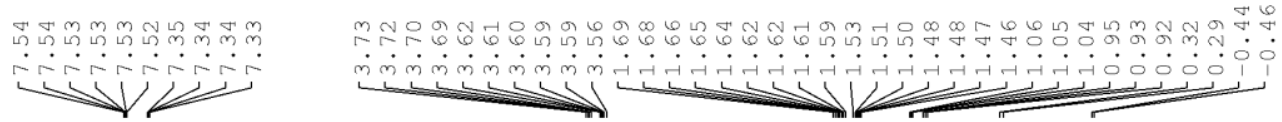

$\therefore$ -
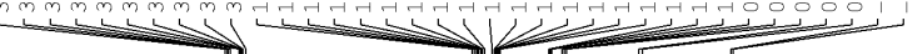
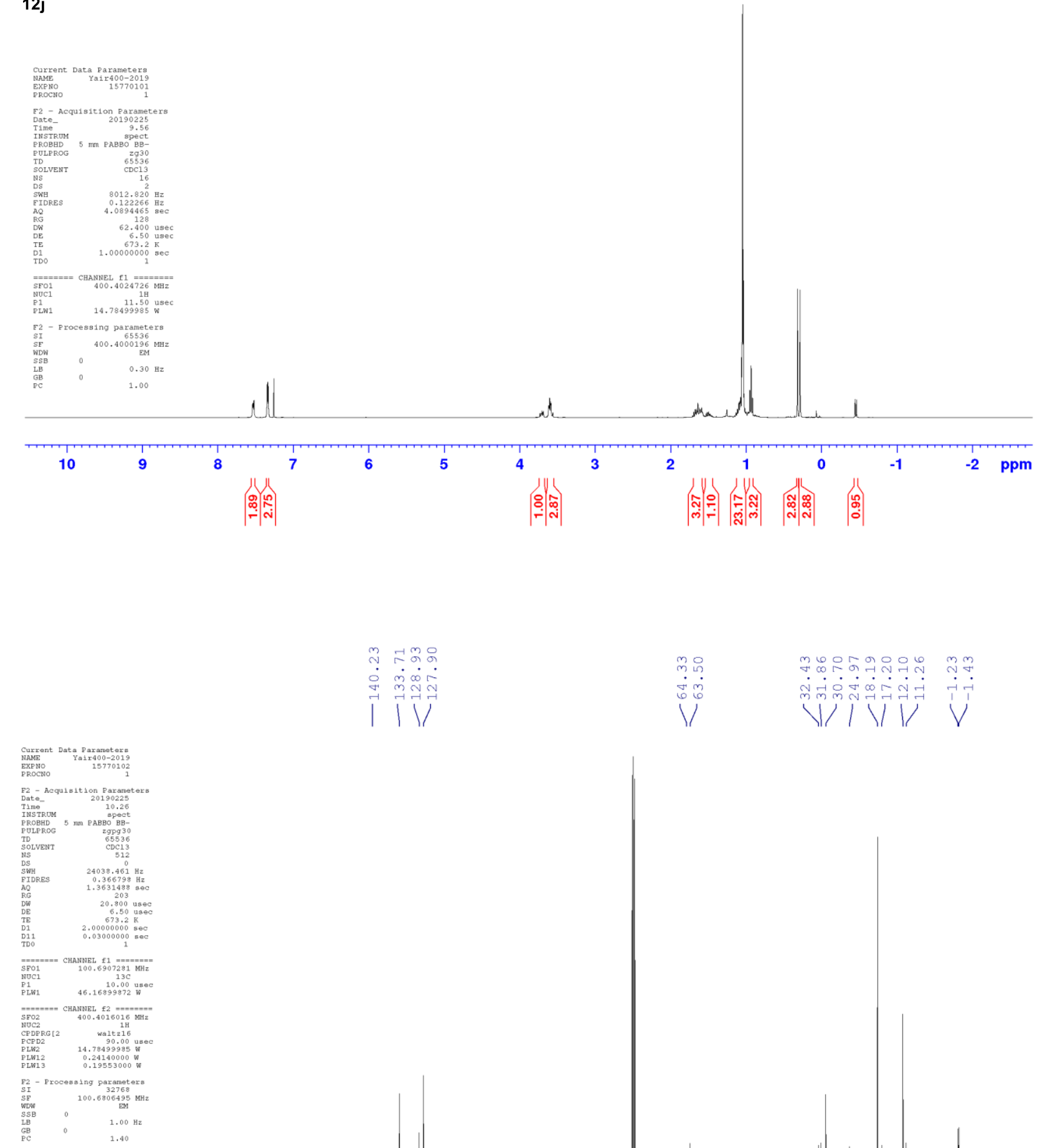

$\dot{7} \dot{2}$
60
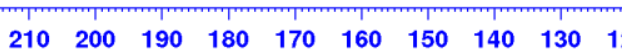

$\begin{array}{lll}10090 & 90\end{array}$
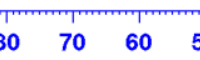


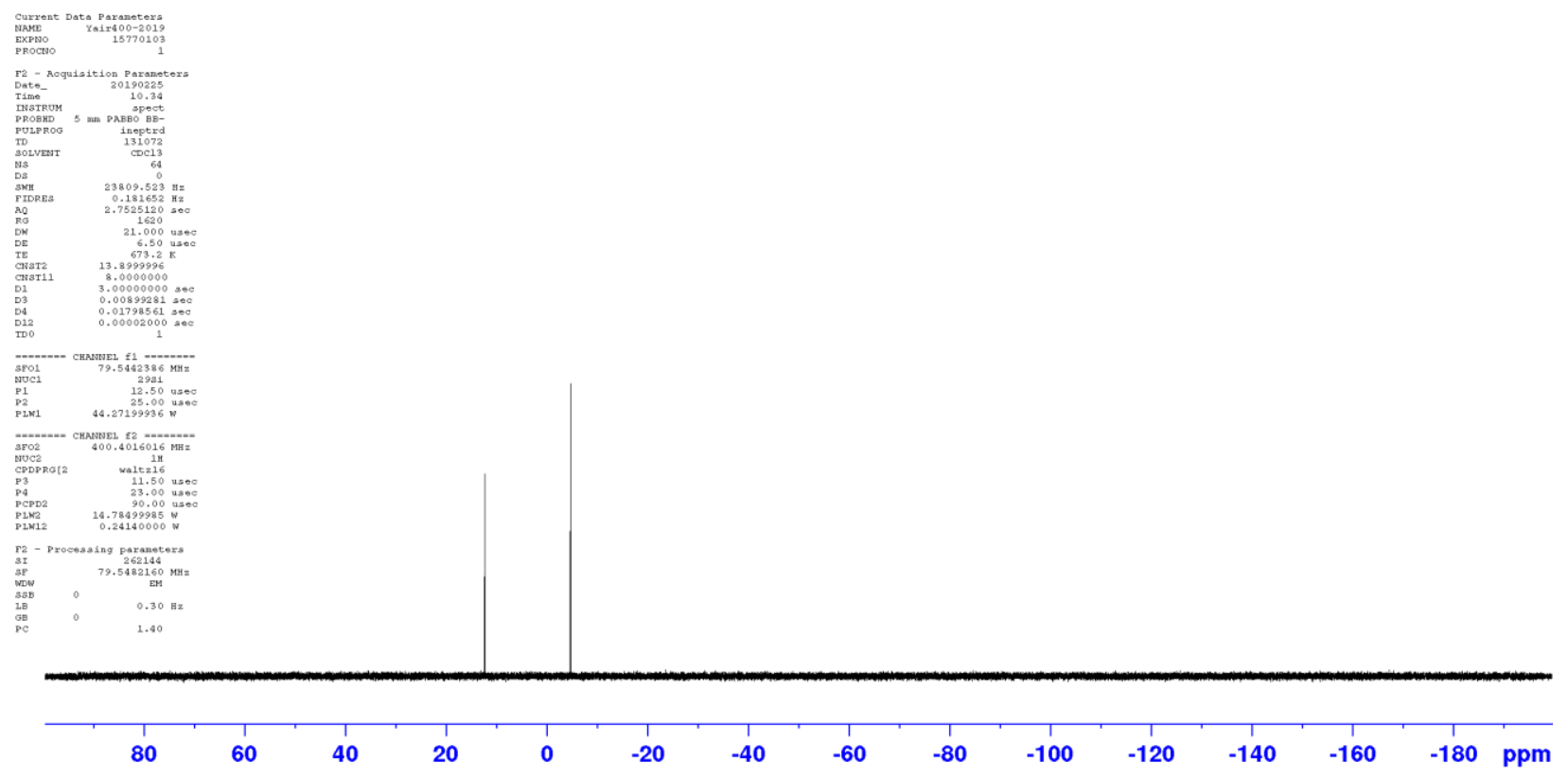



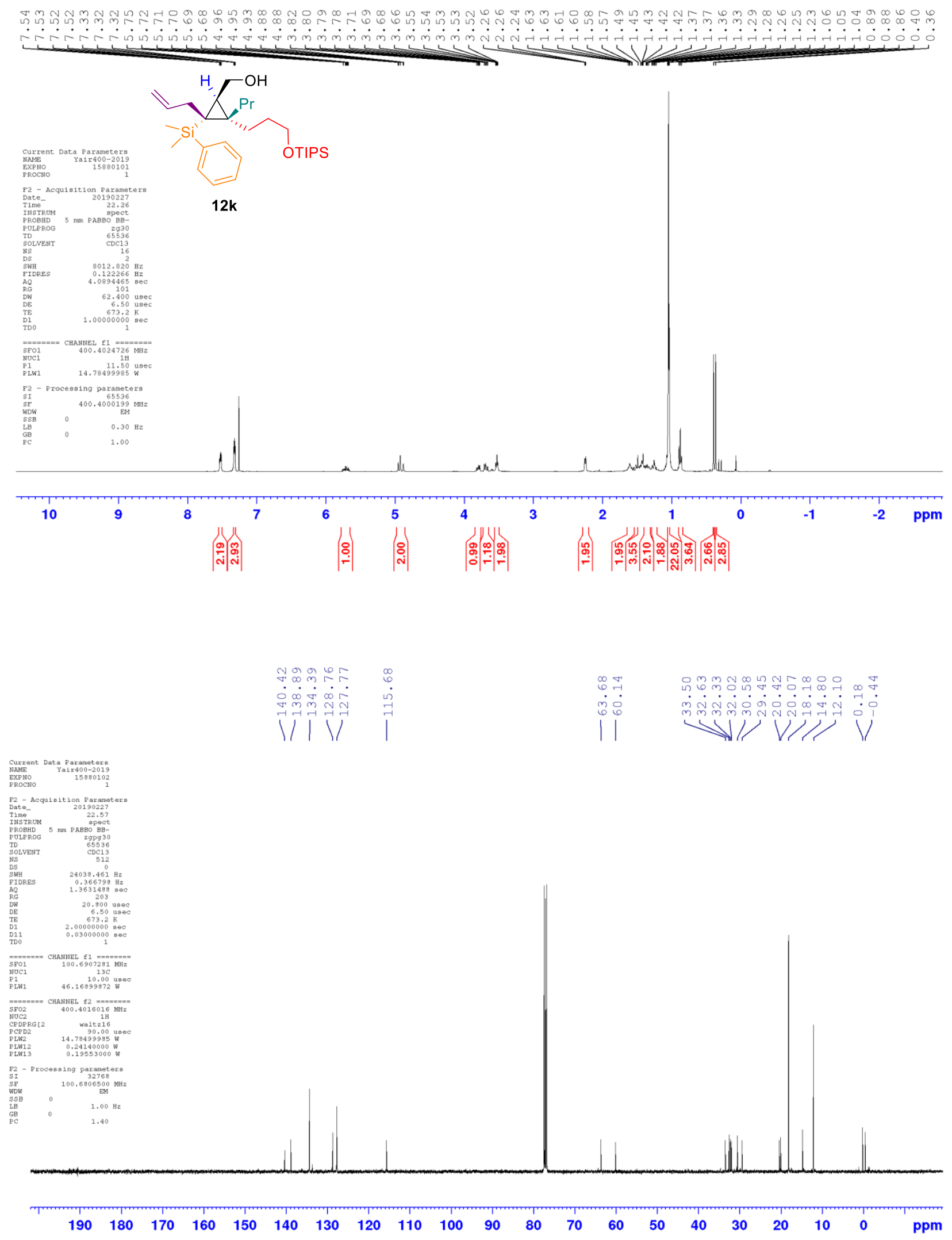


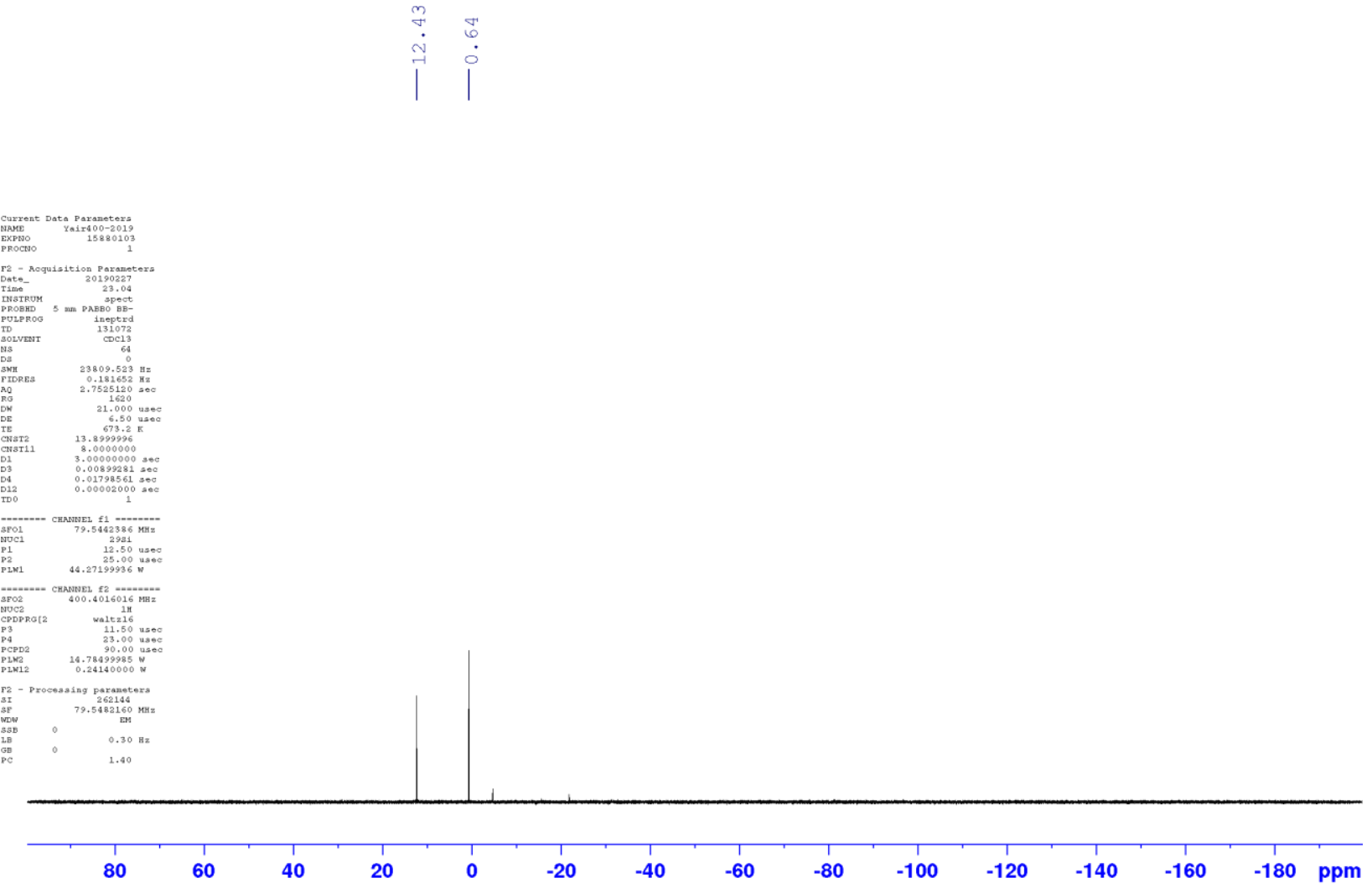




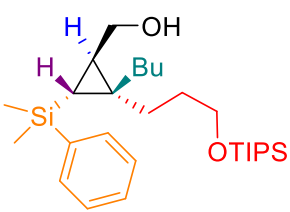

121

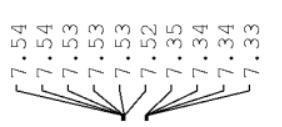

Nㅇำ

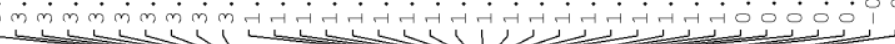

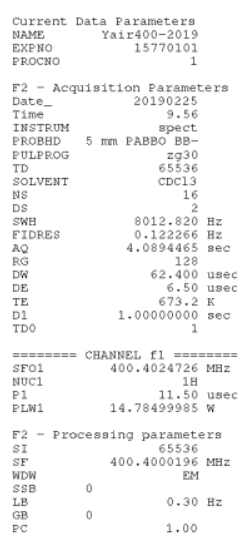

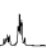

Wa.
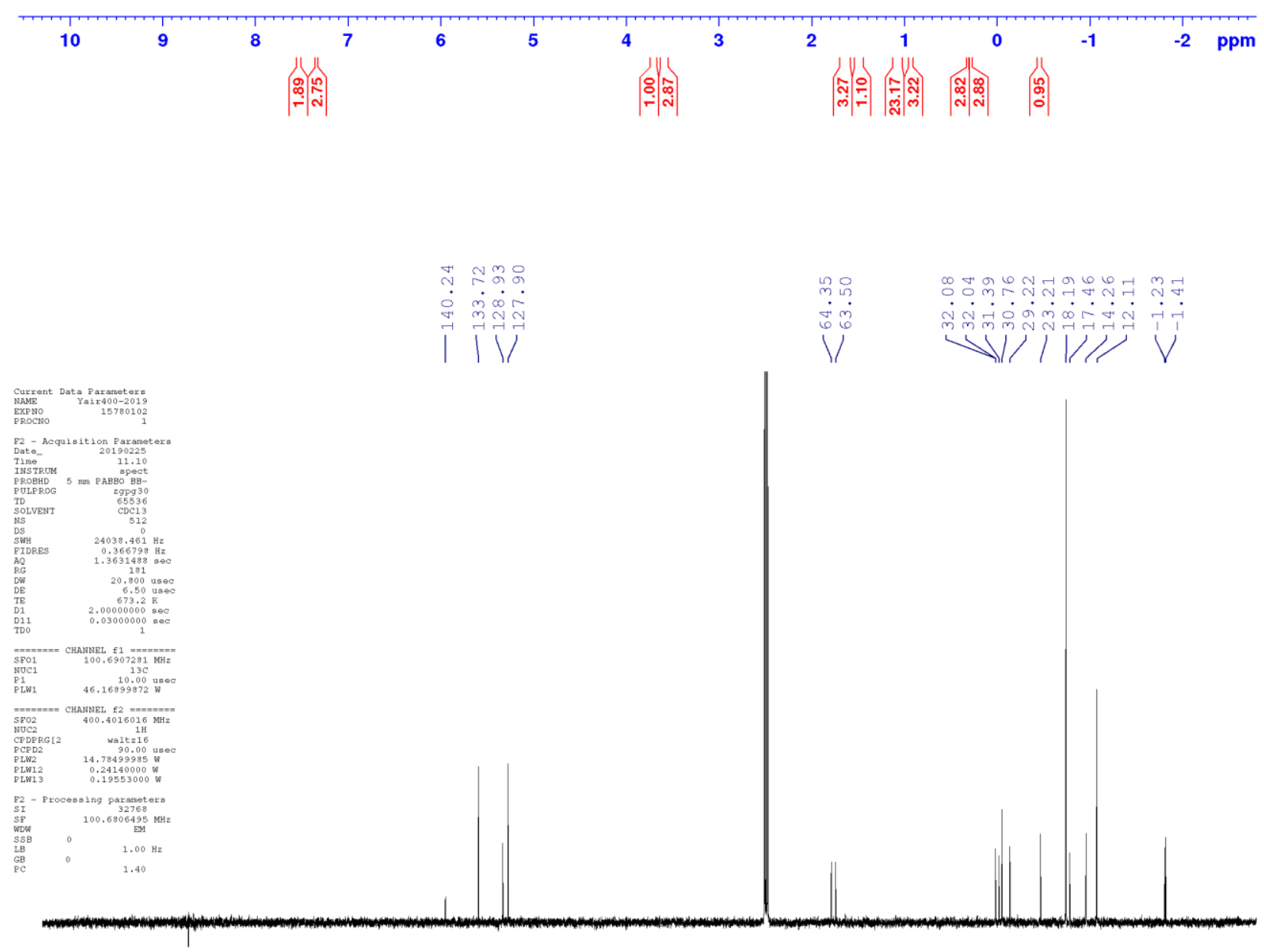

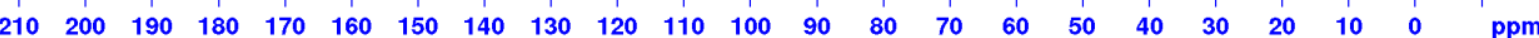




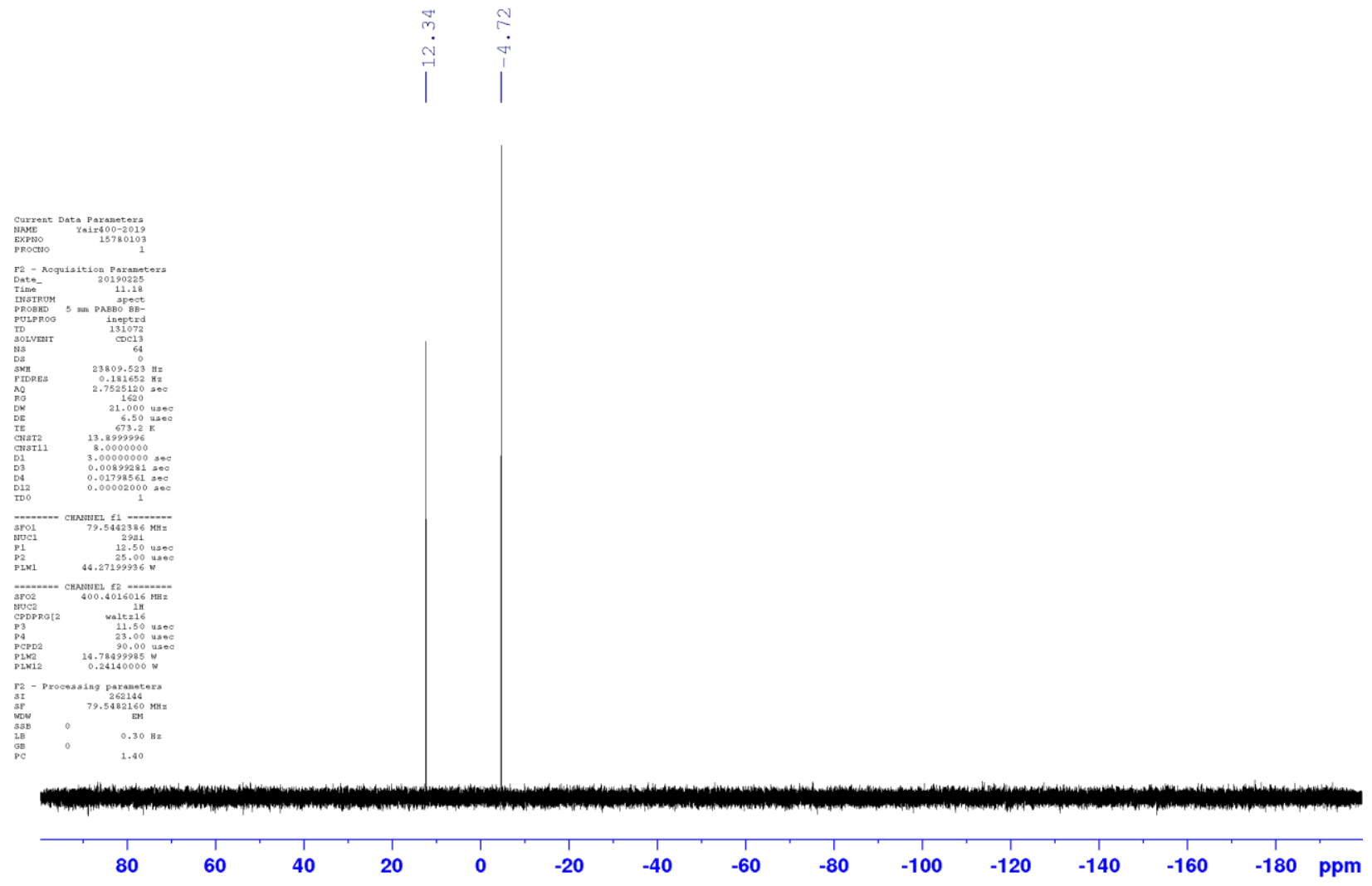



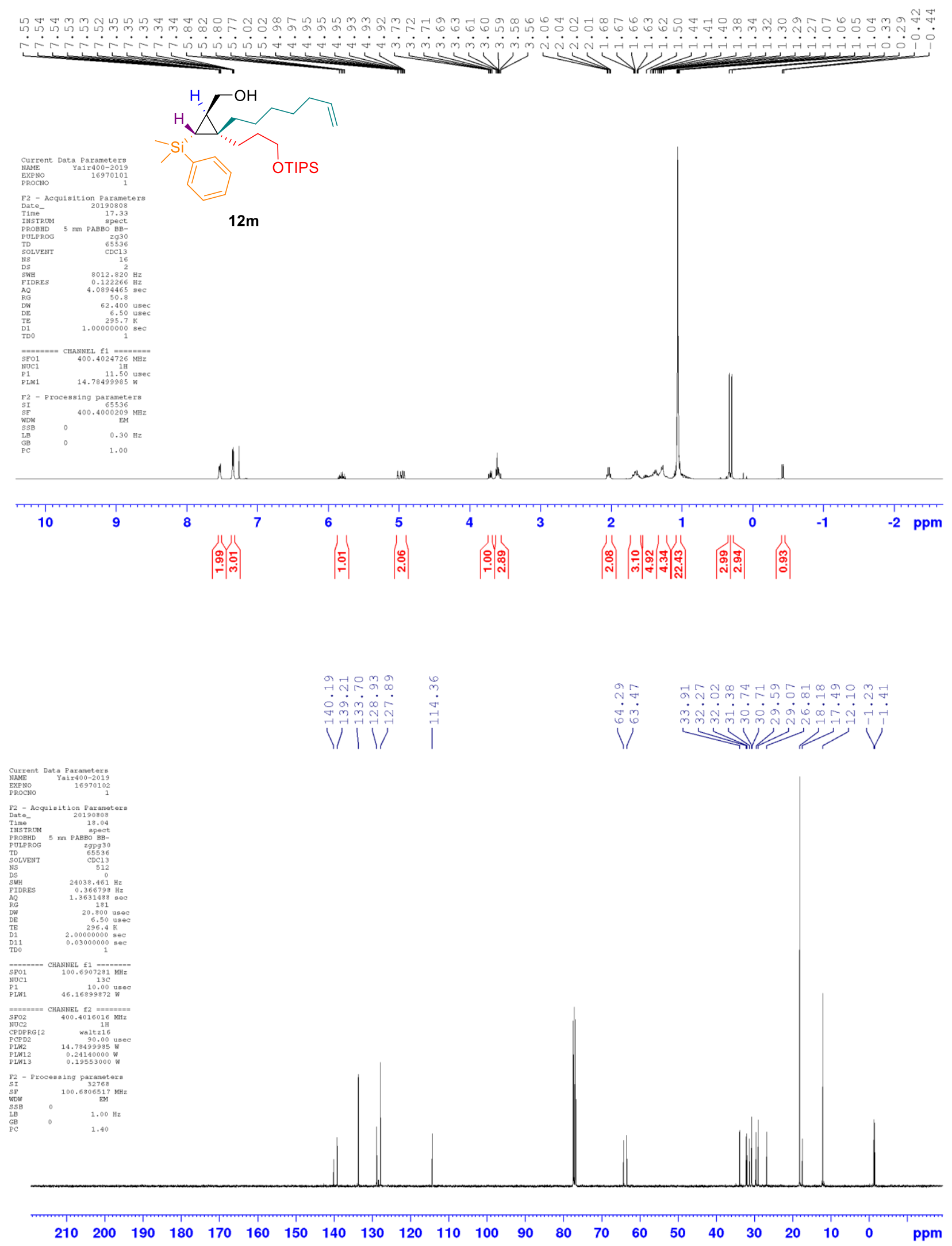


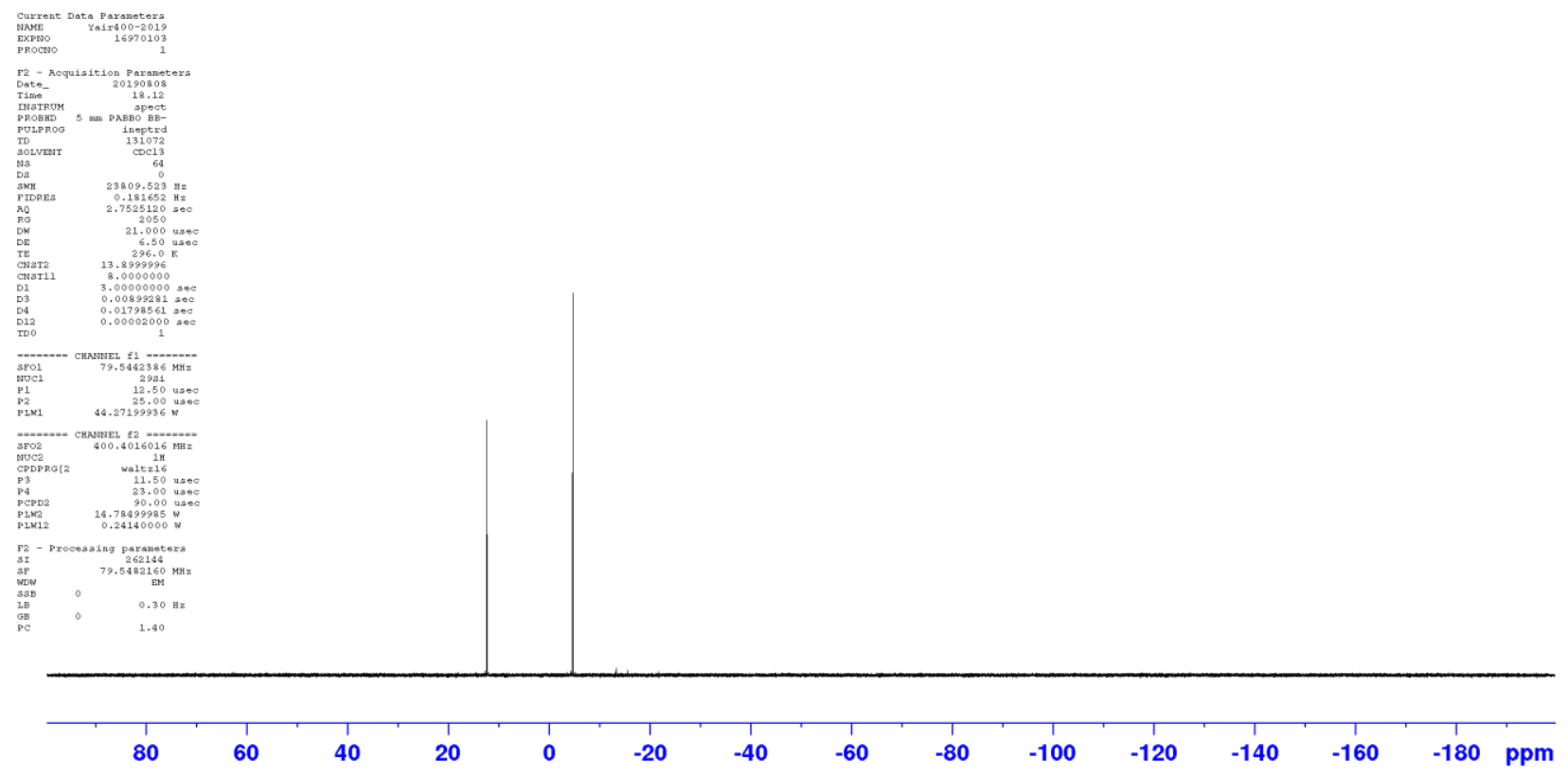



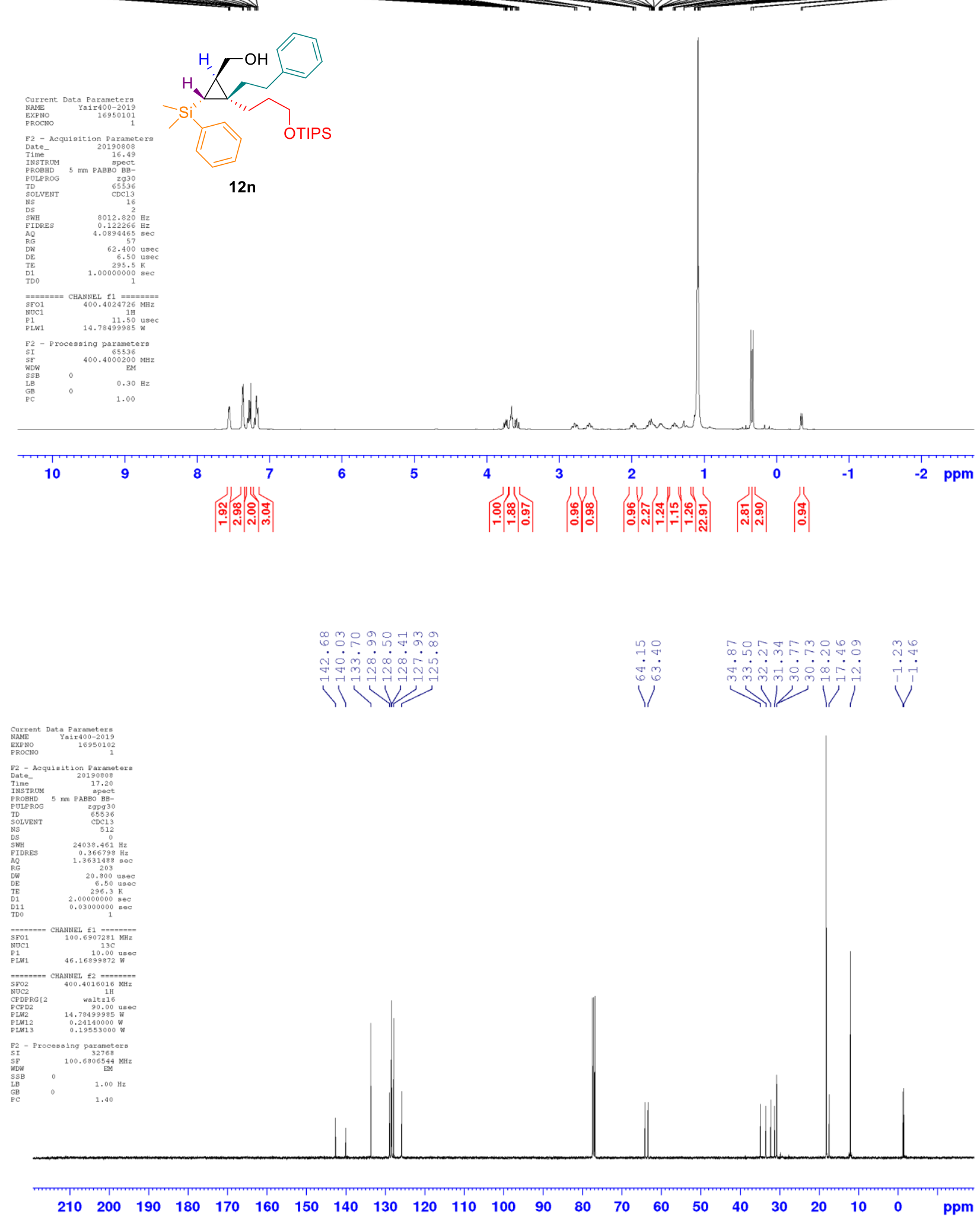


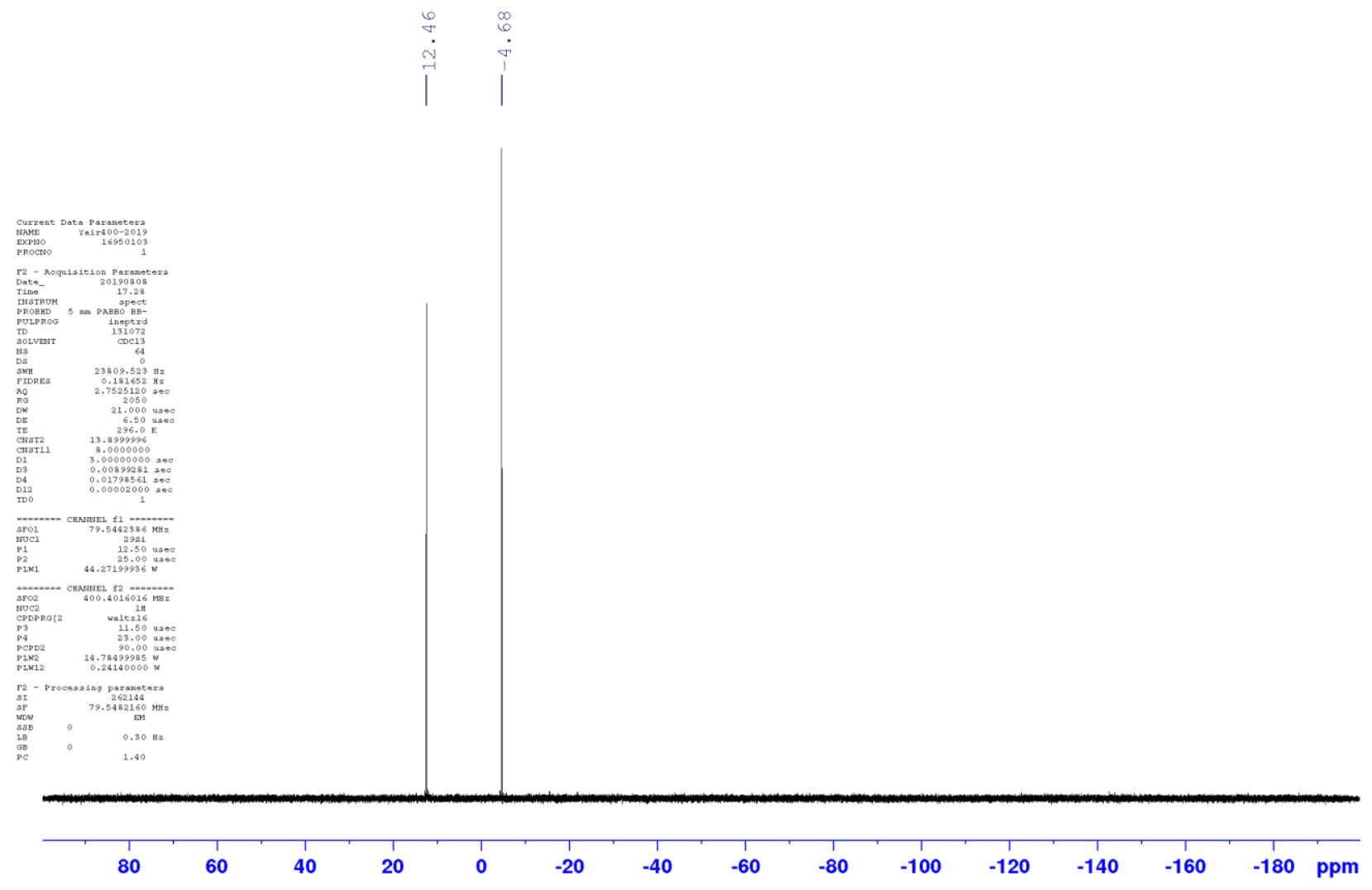




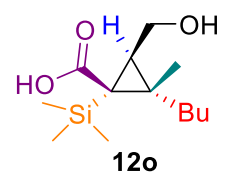

120
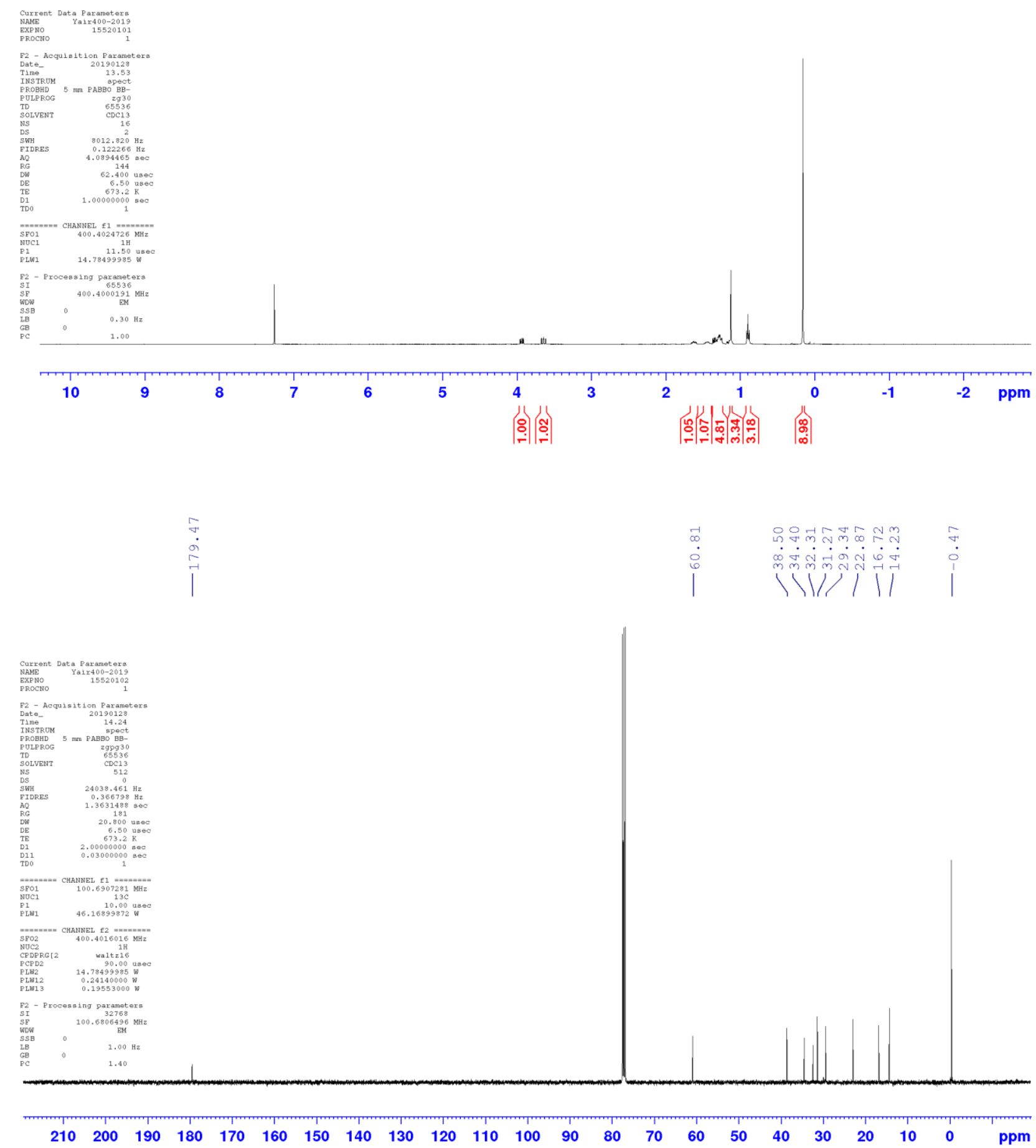


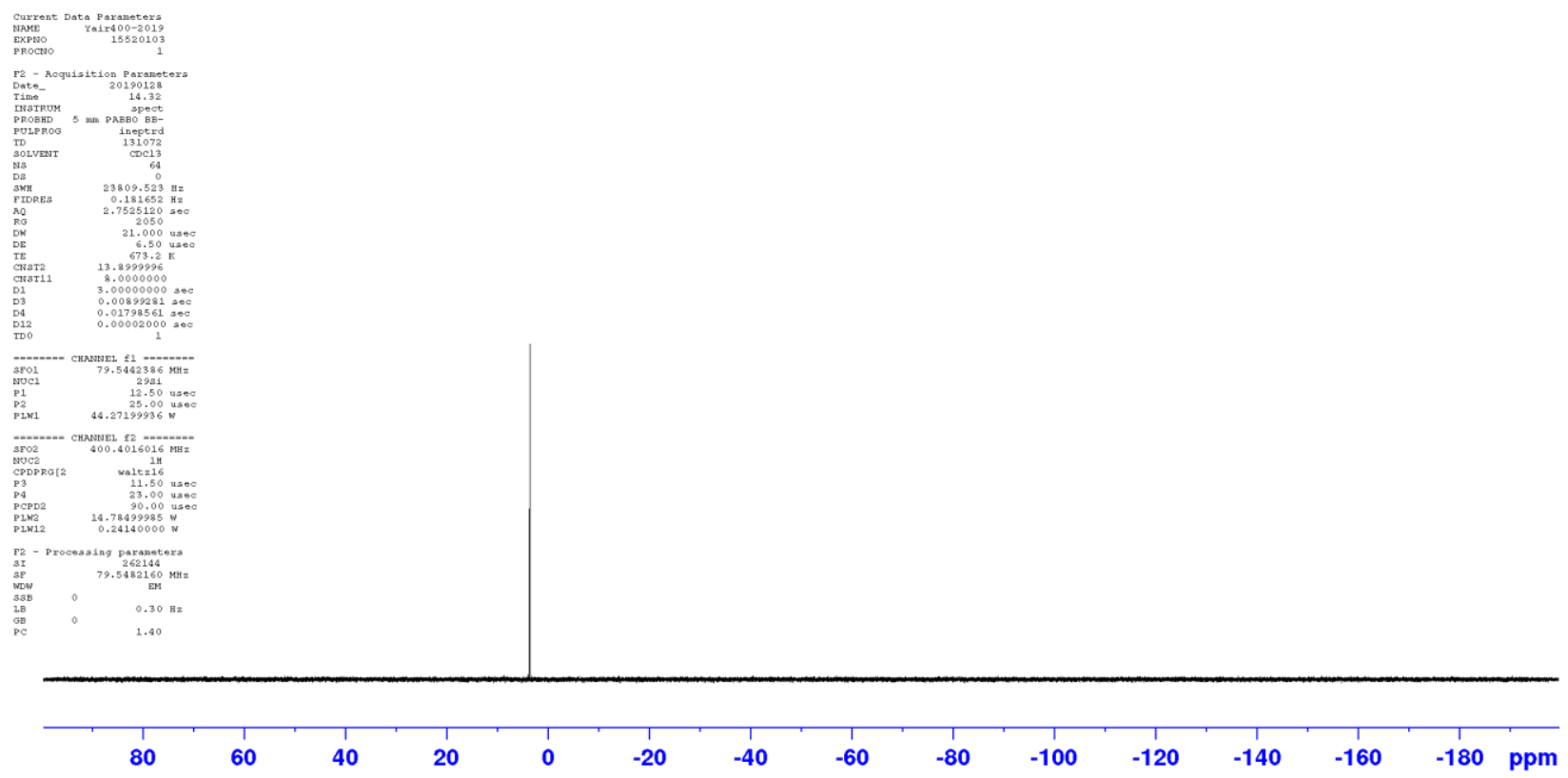




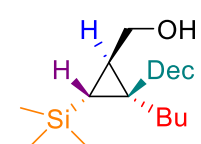

$12 p$

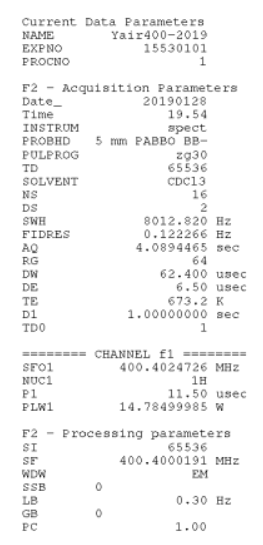

dit

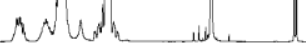

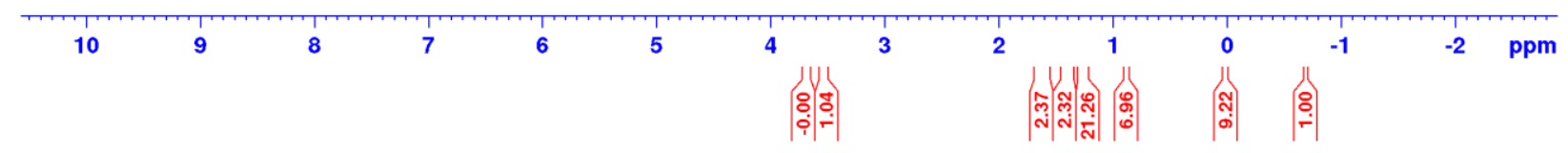

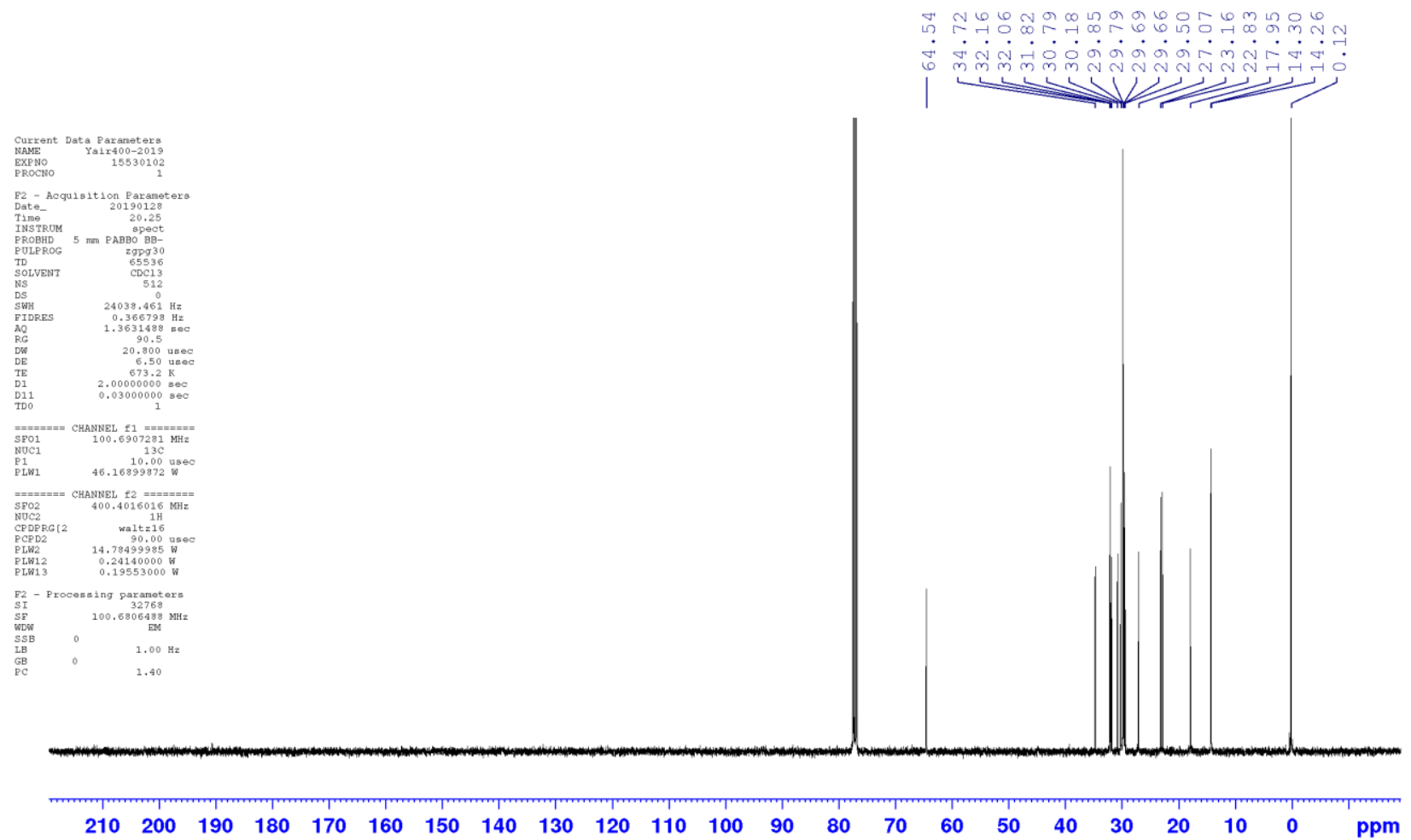




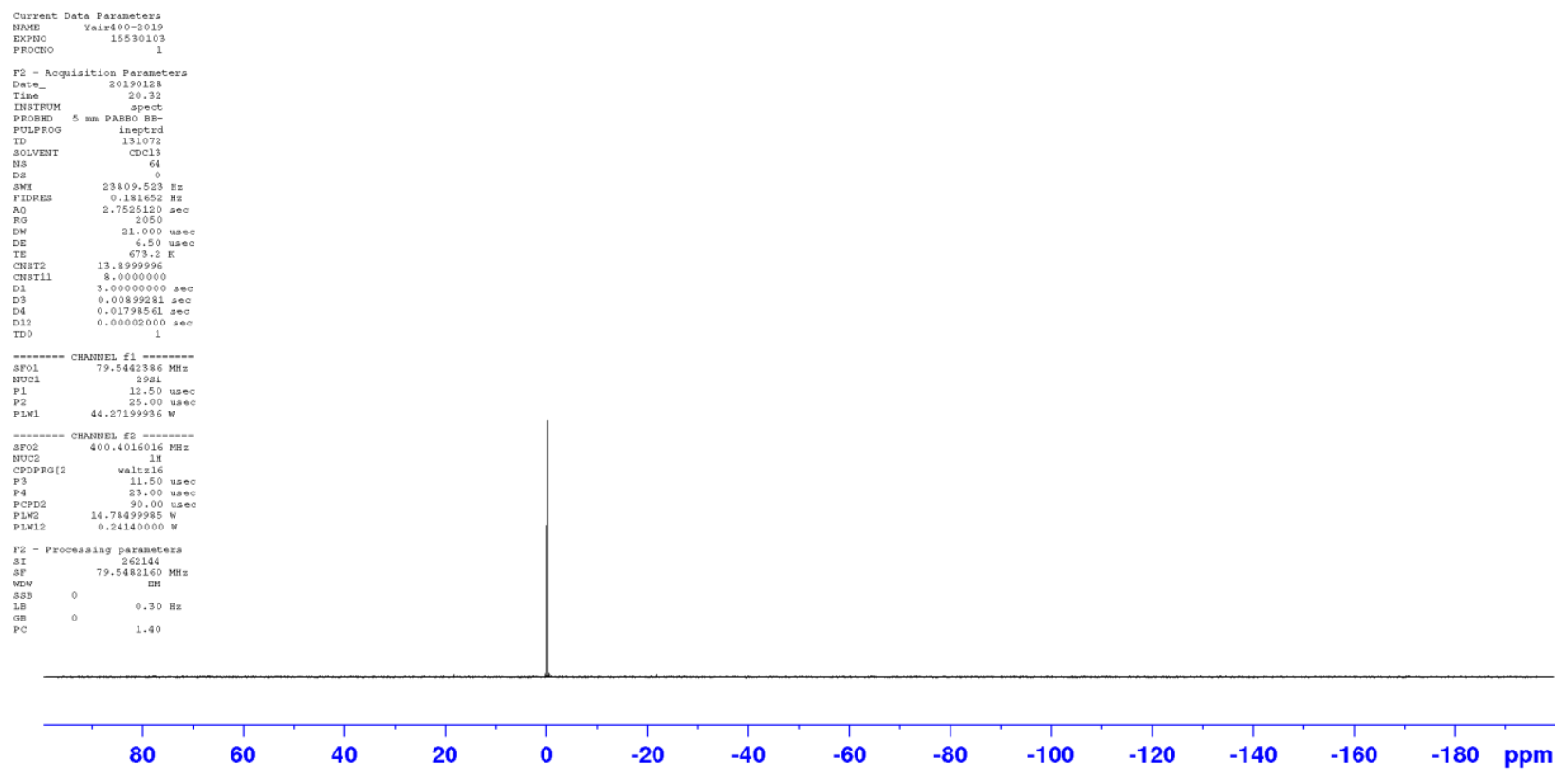



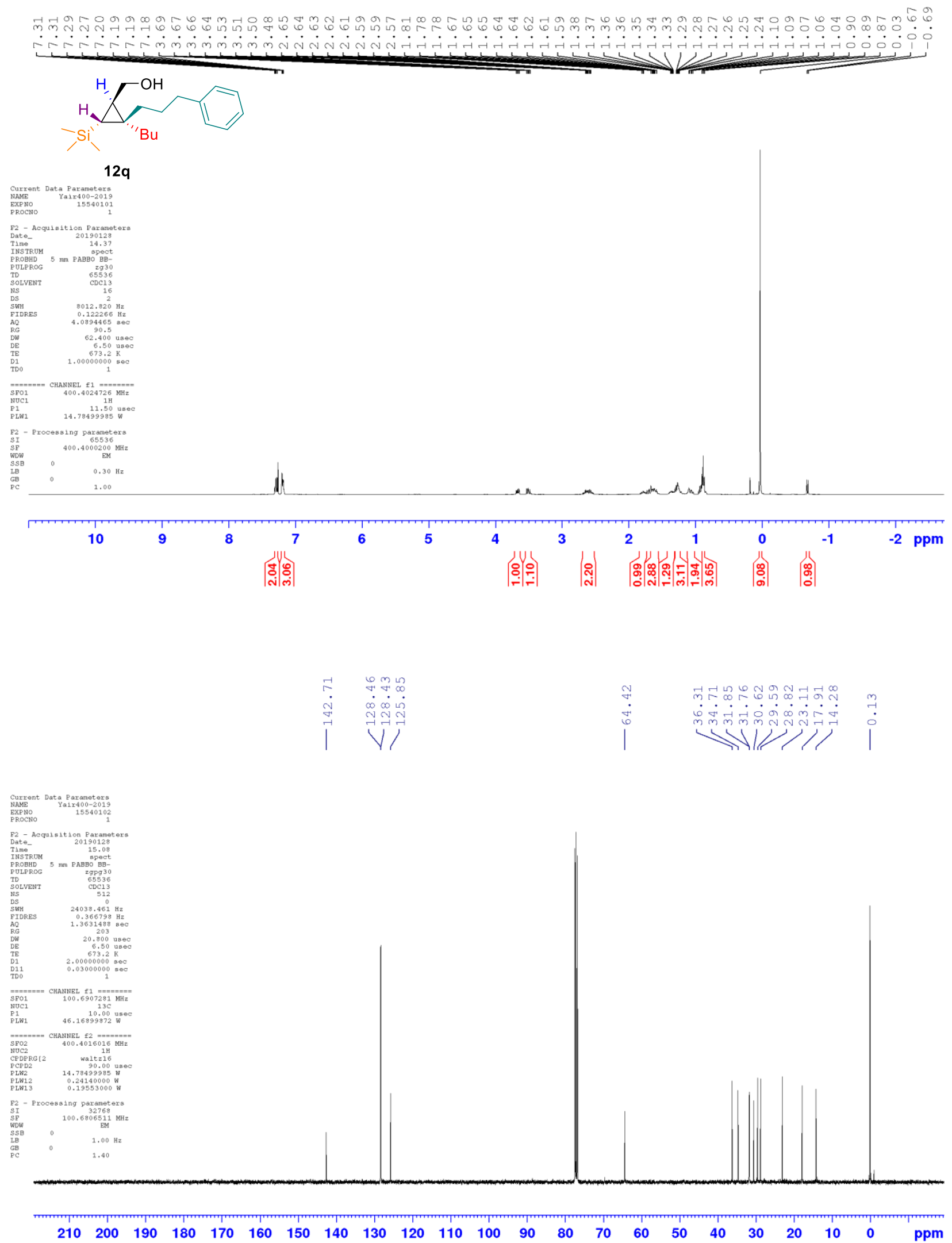


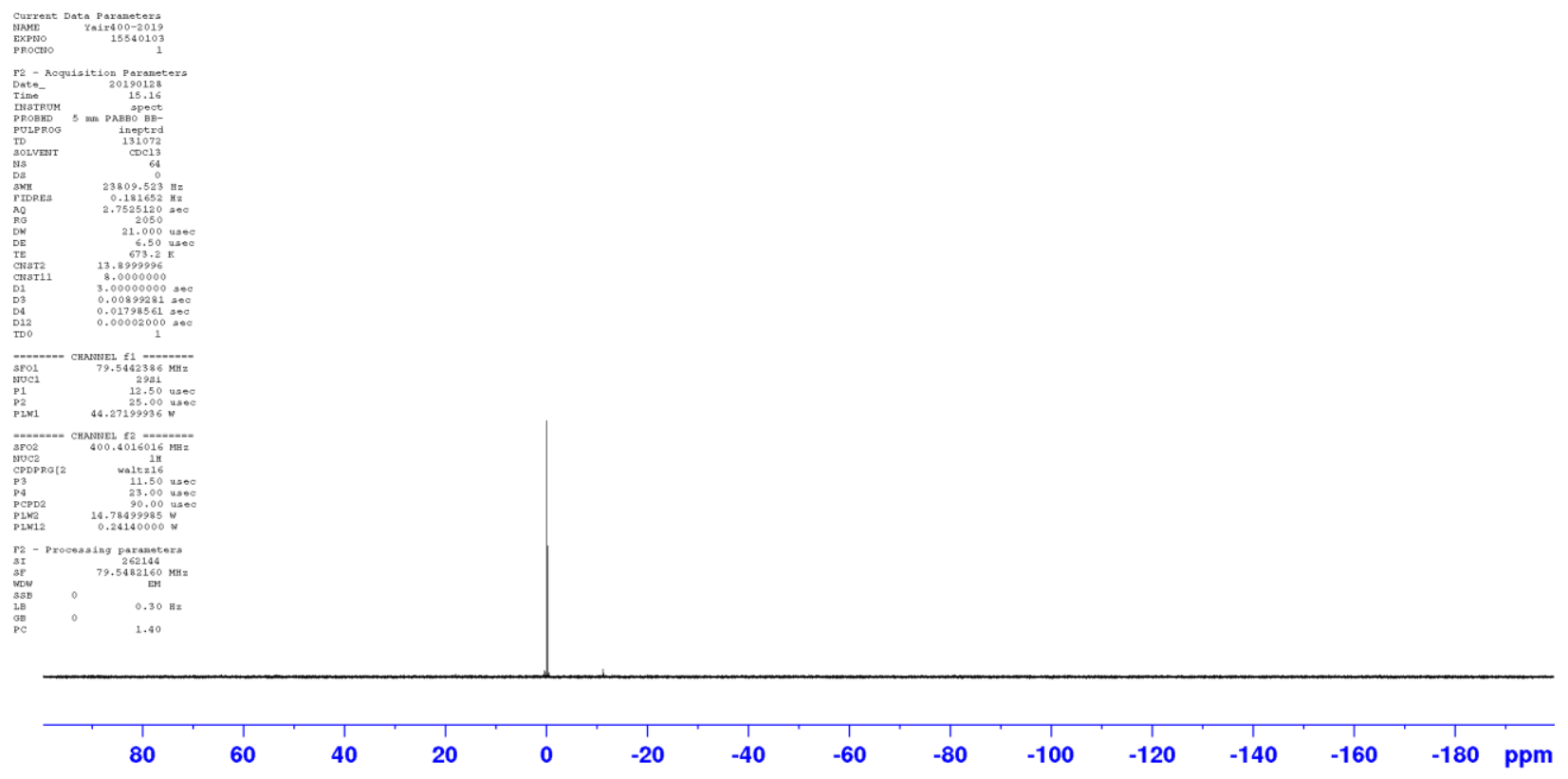



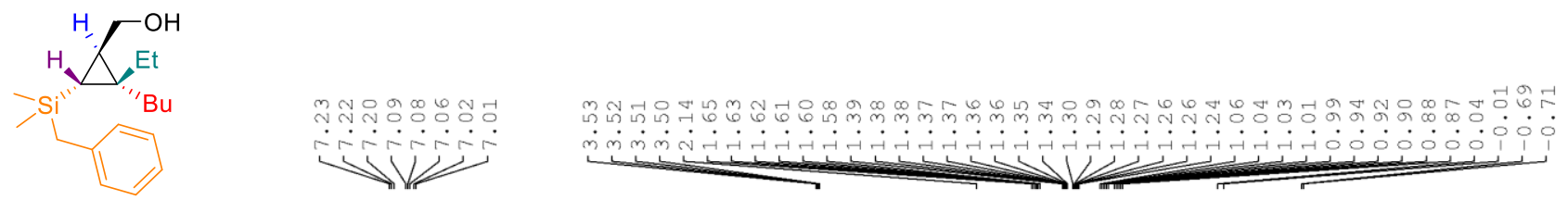

$12 r$
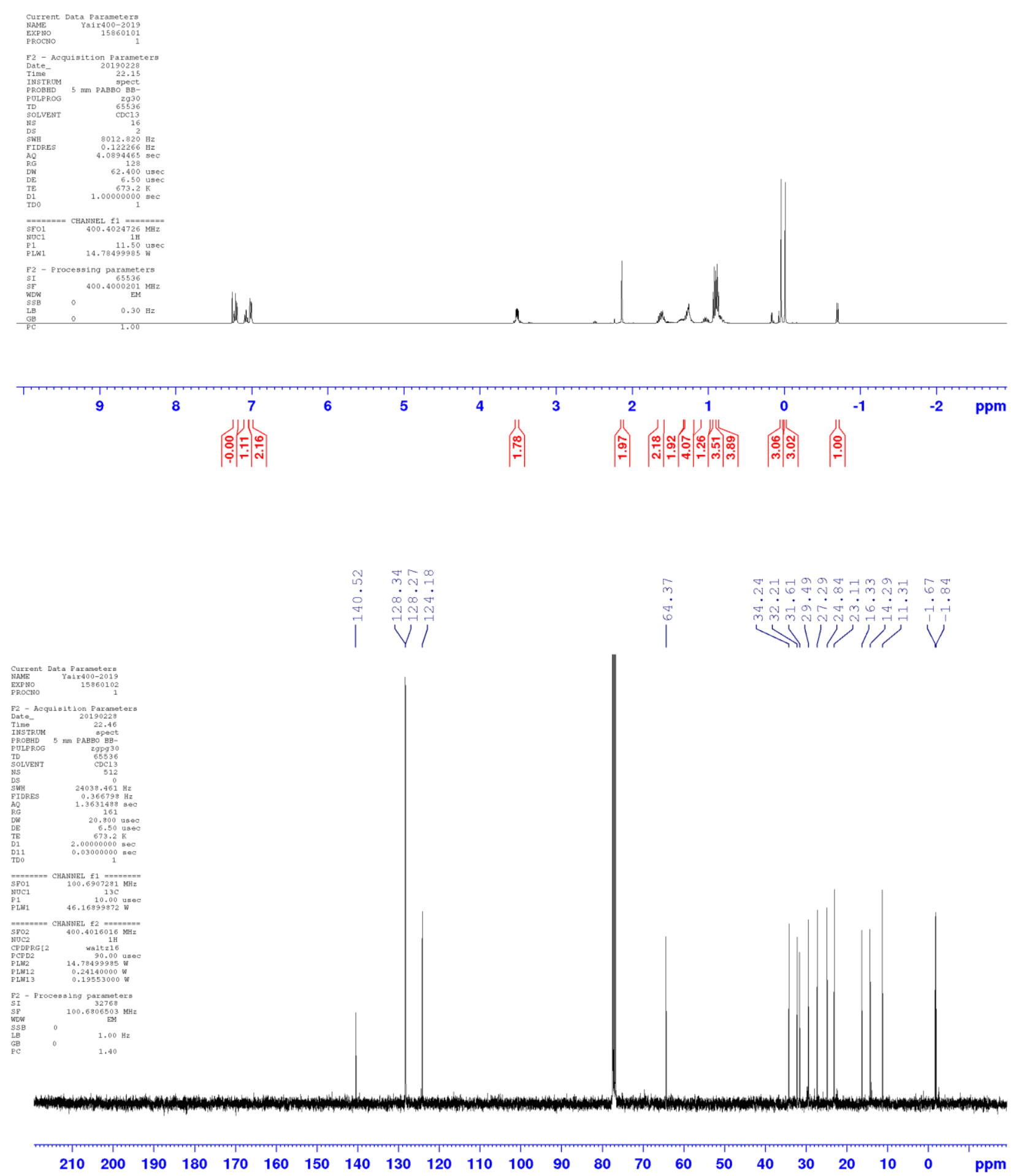


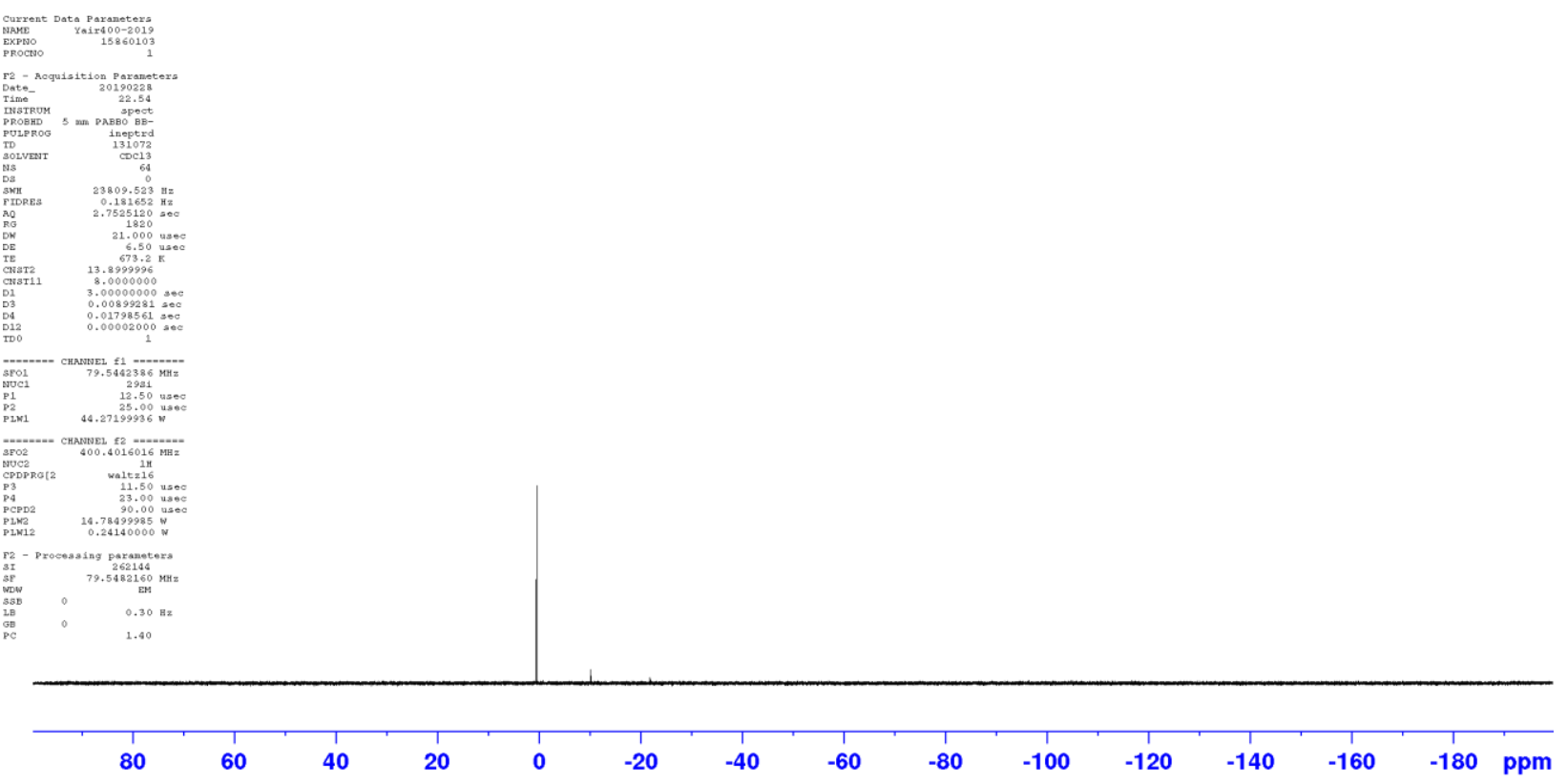




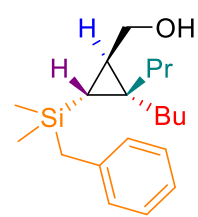

$12 s$

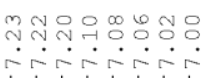

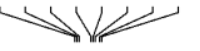

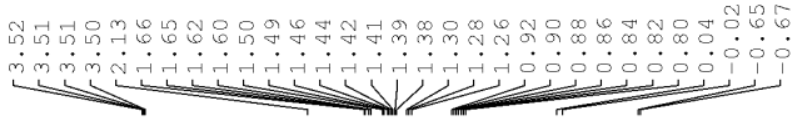
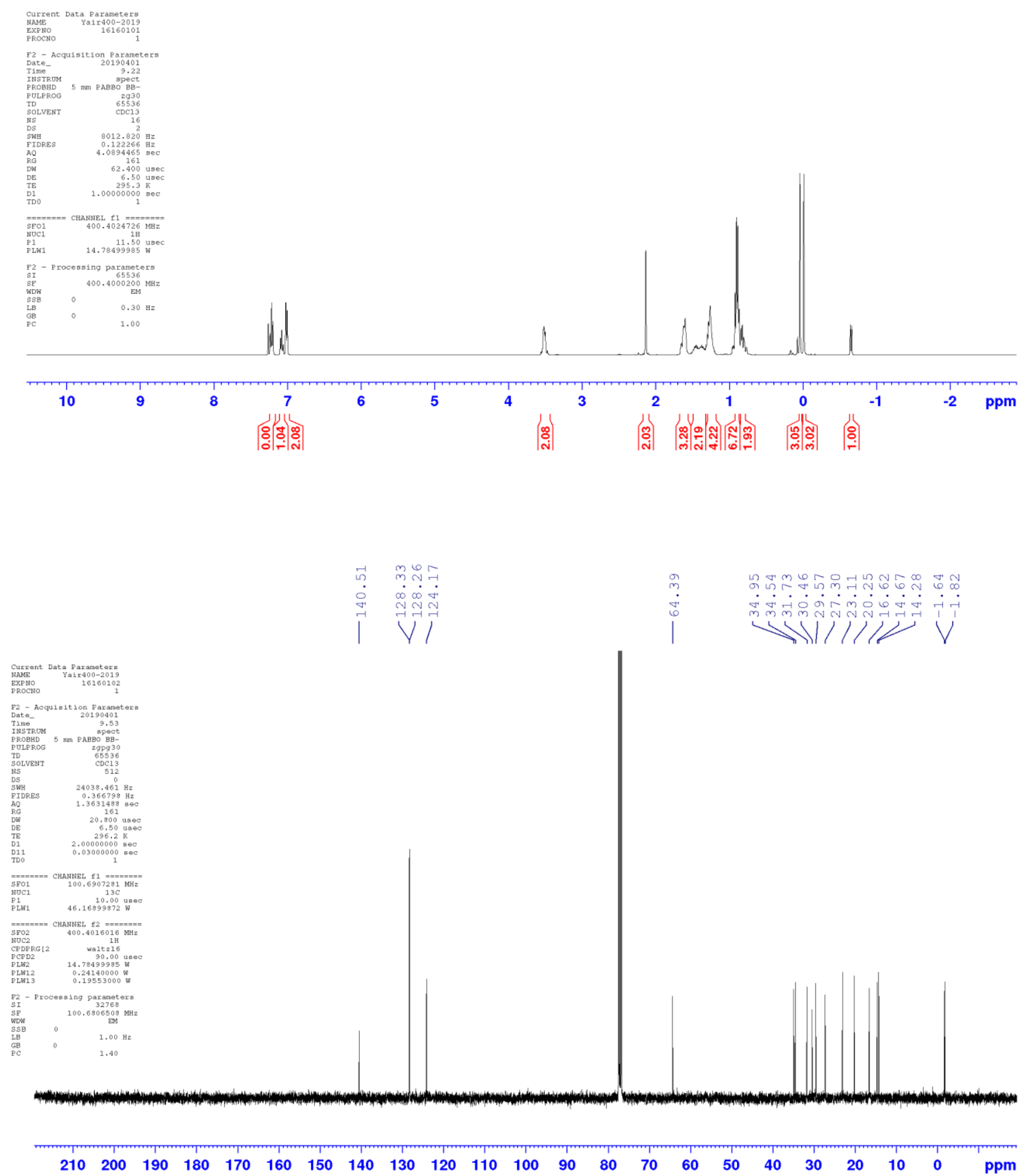


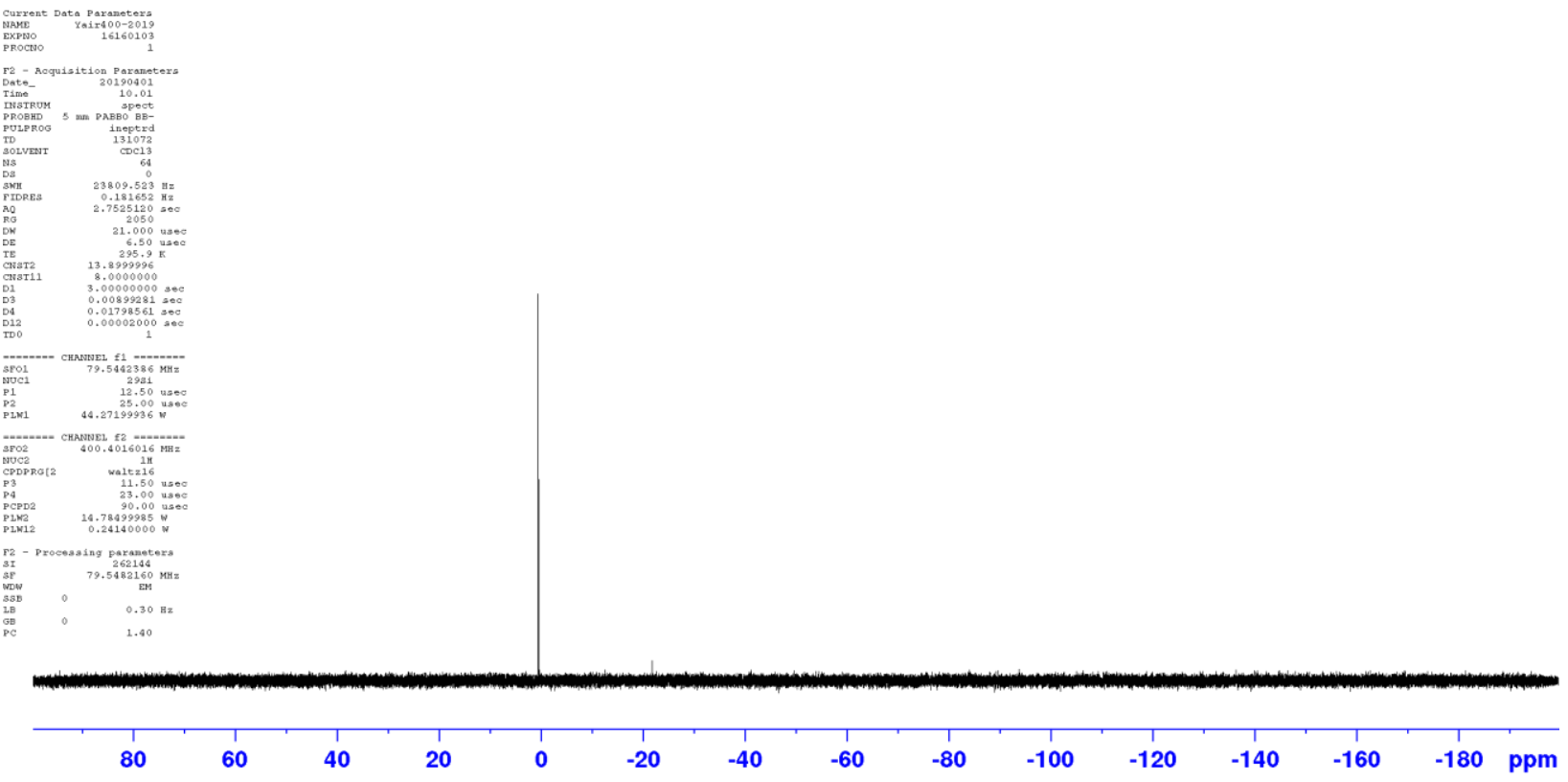




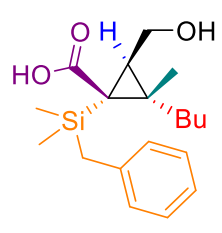

$12 t$
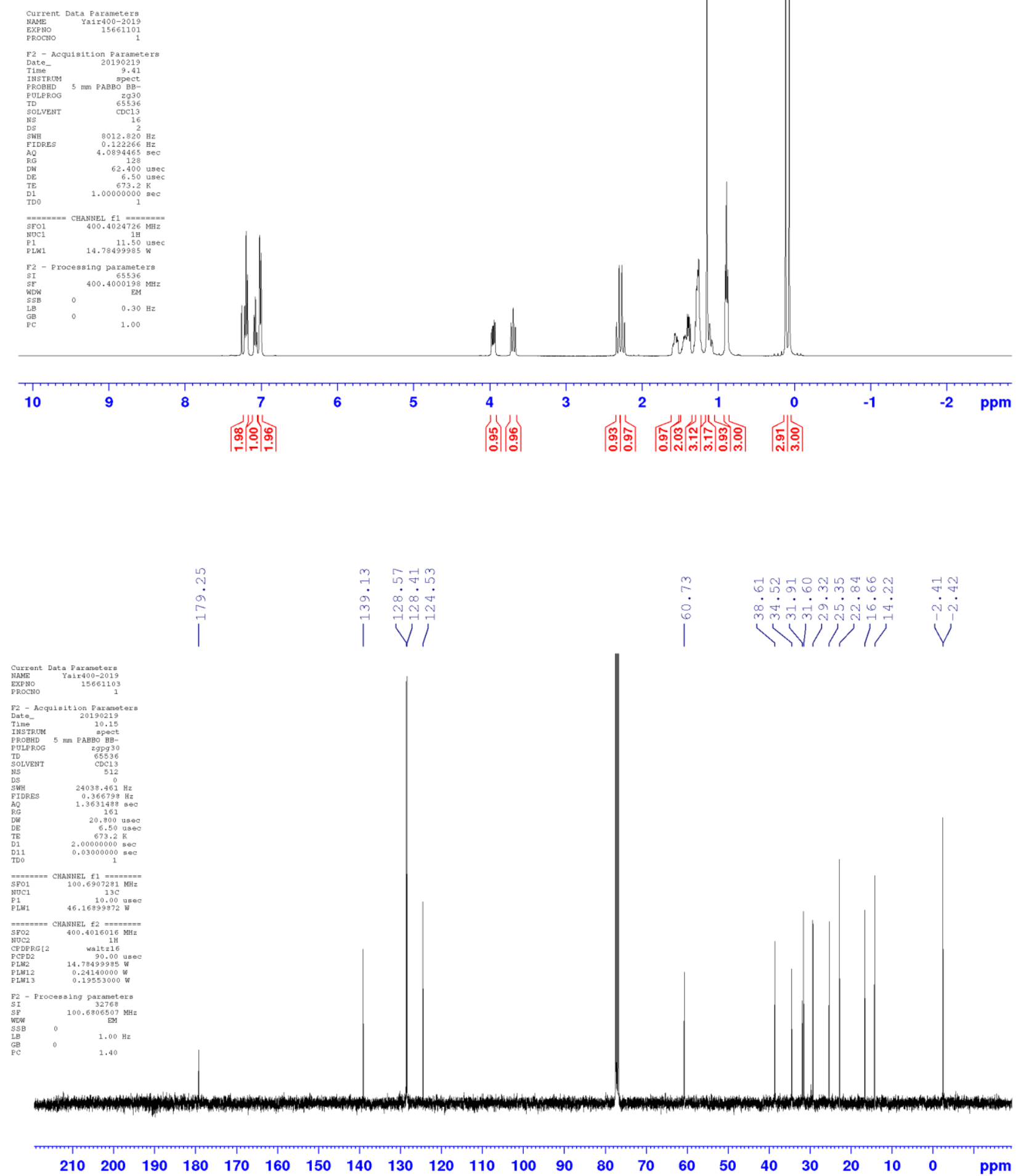


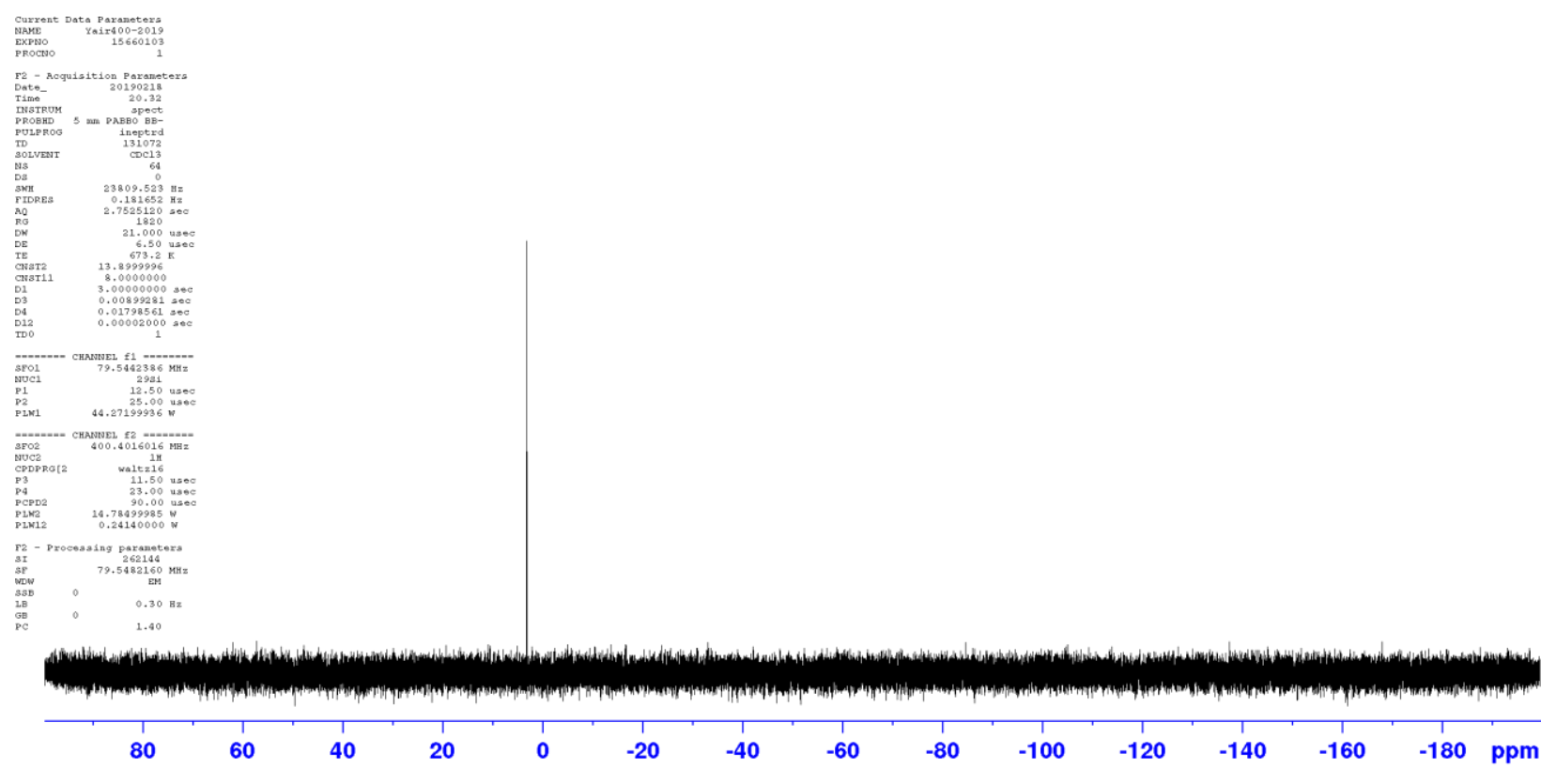



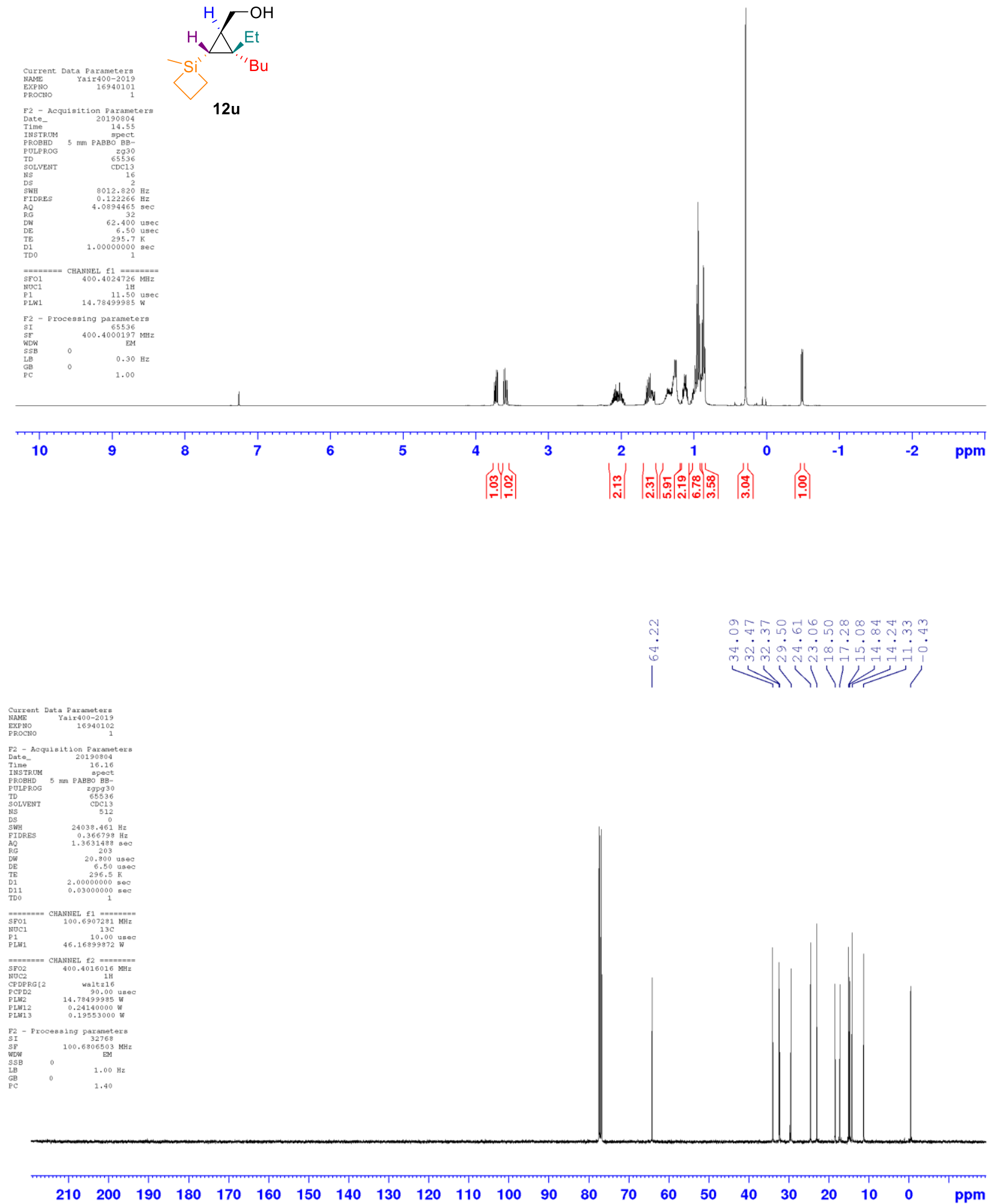


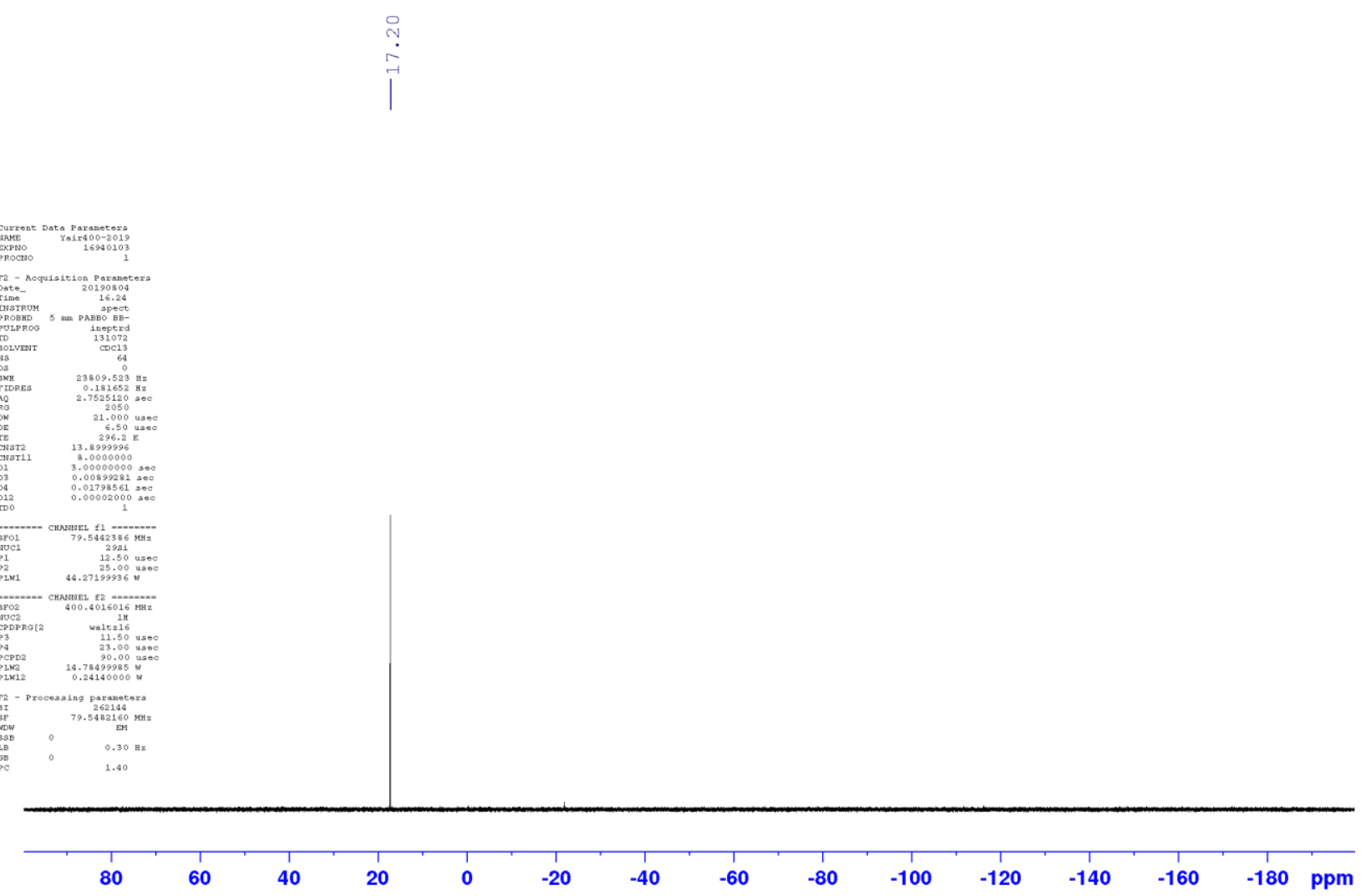



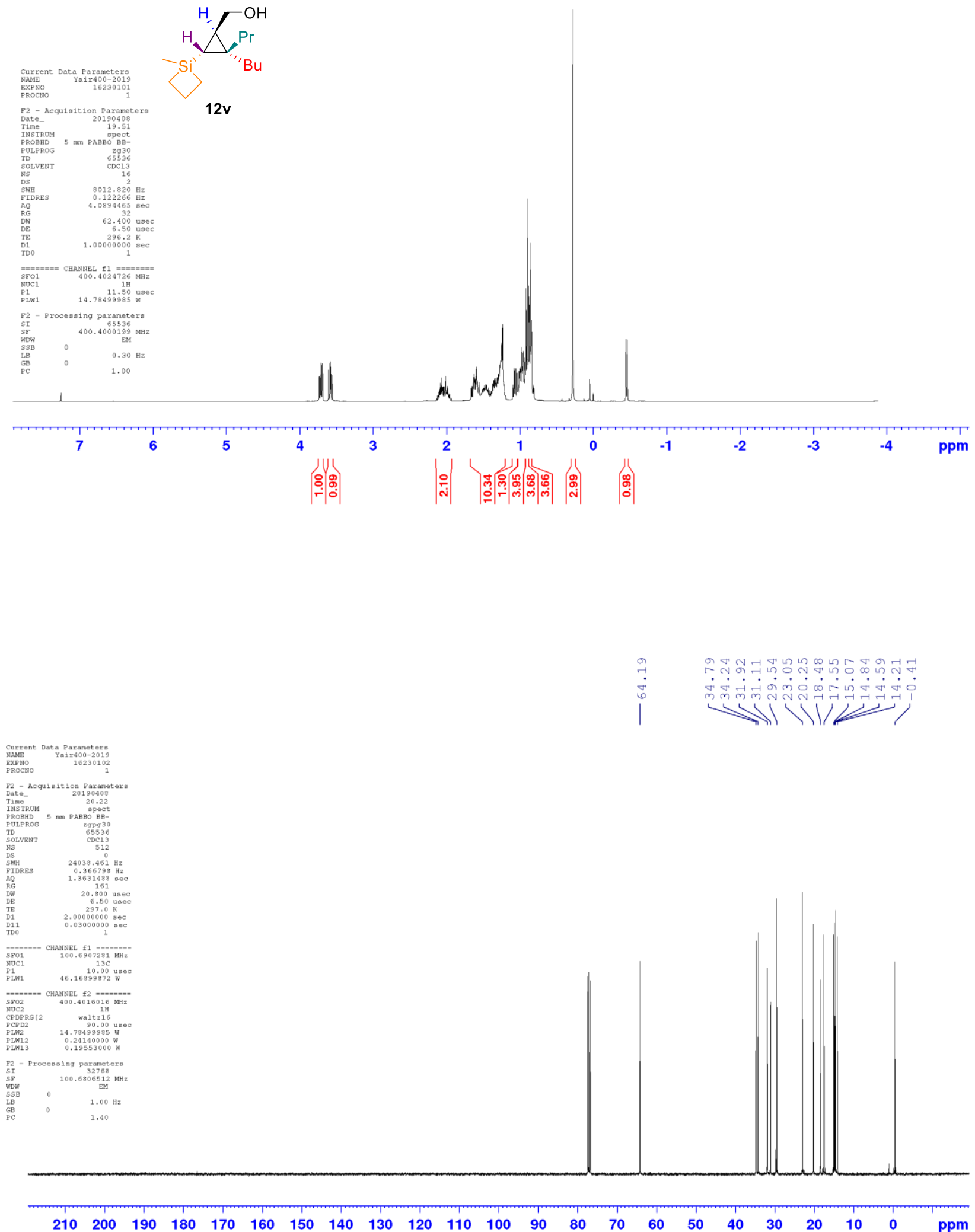


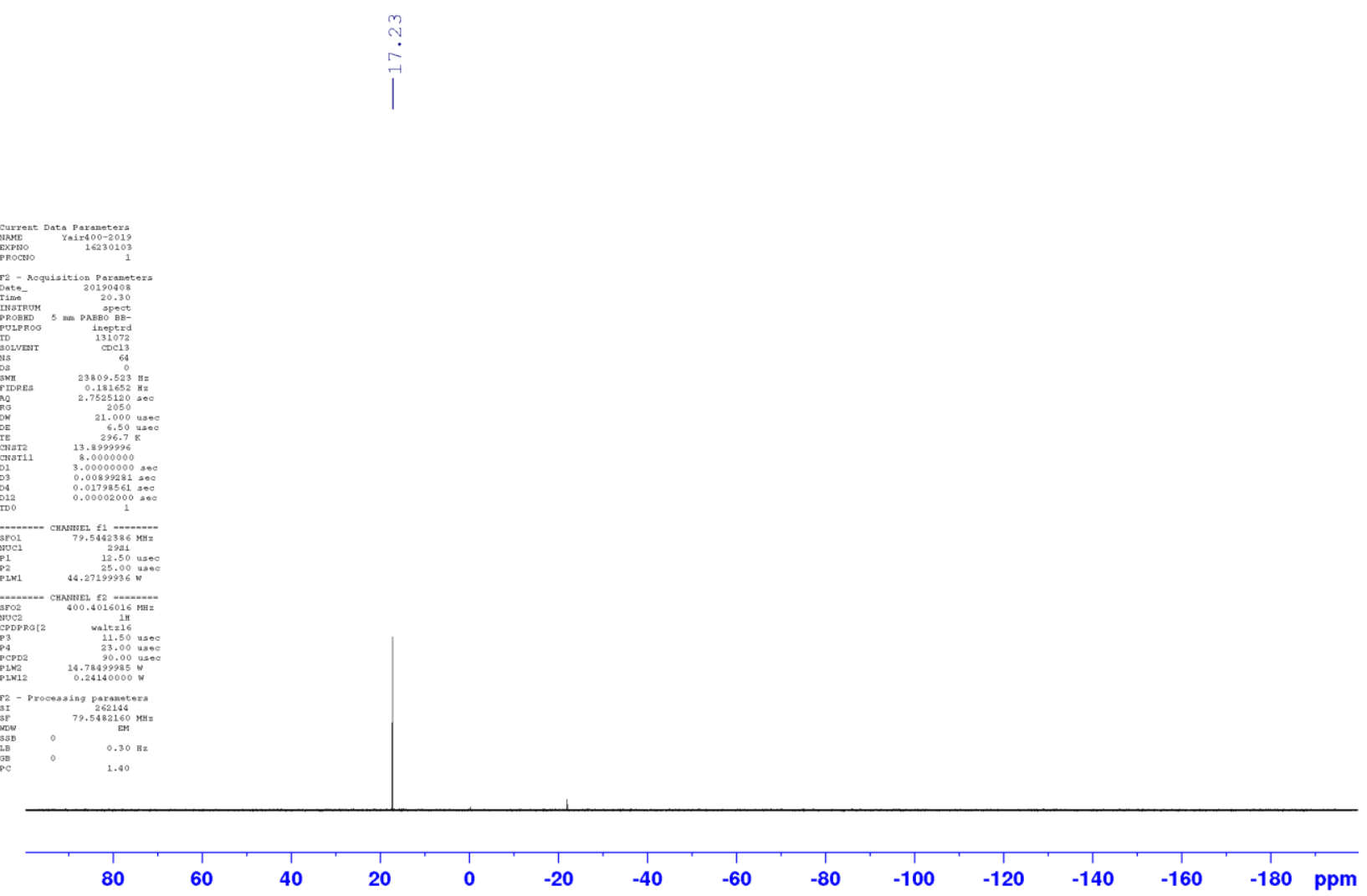



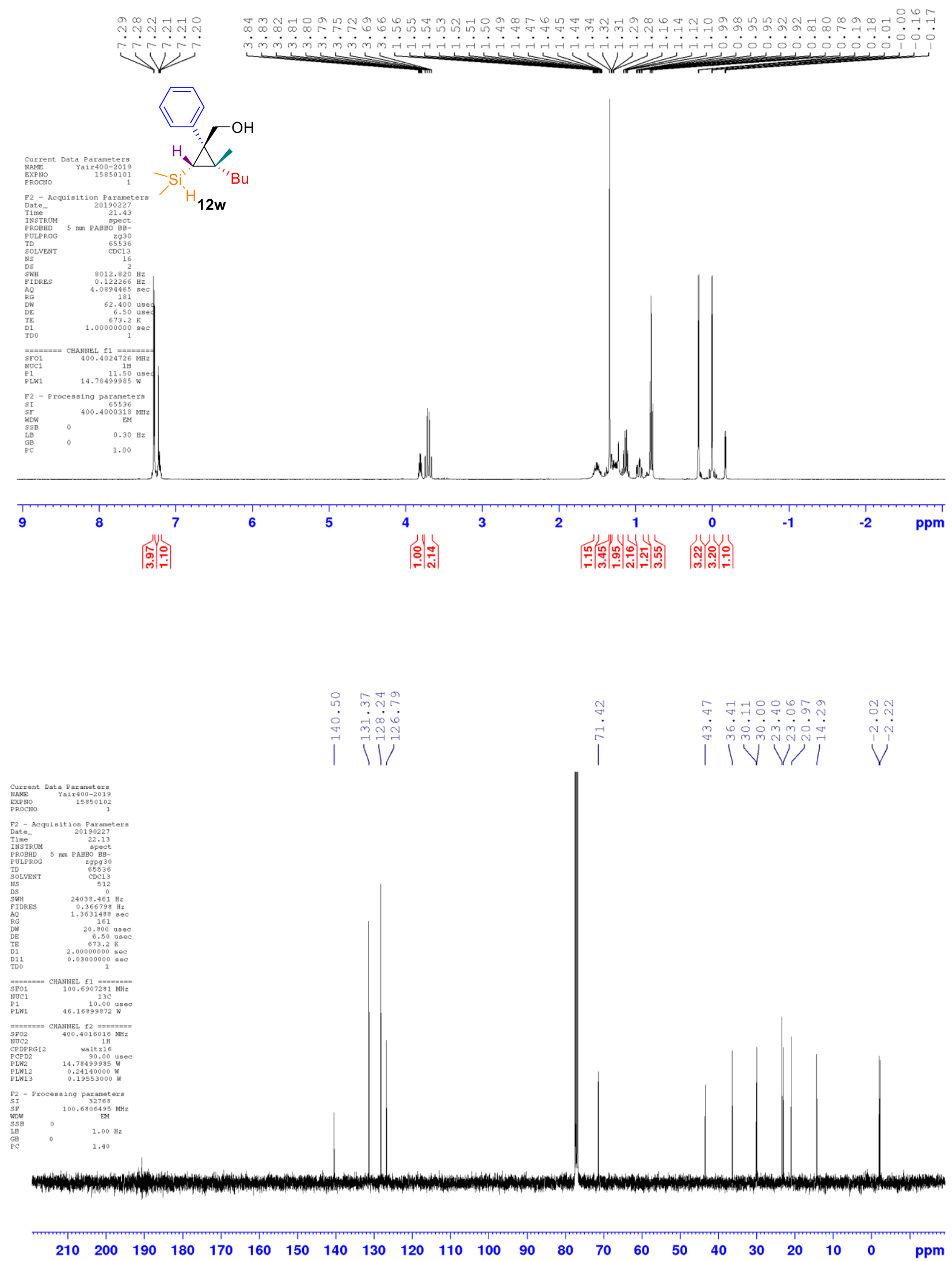


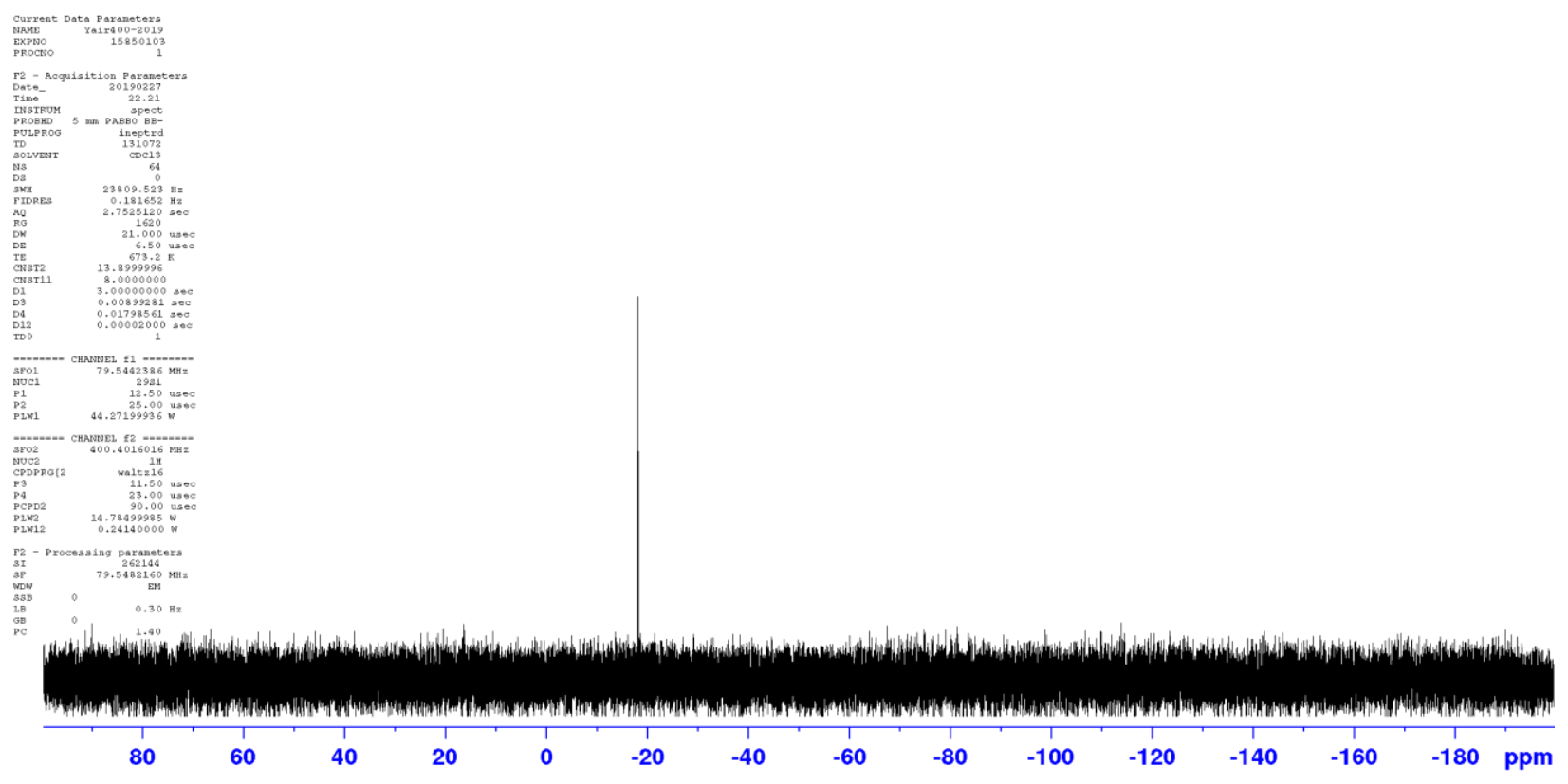



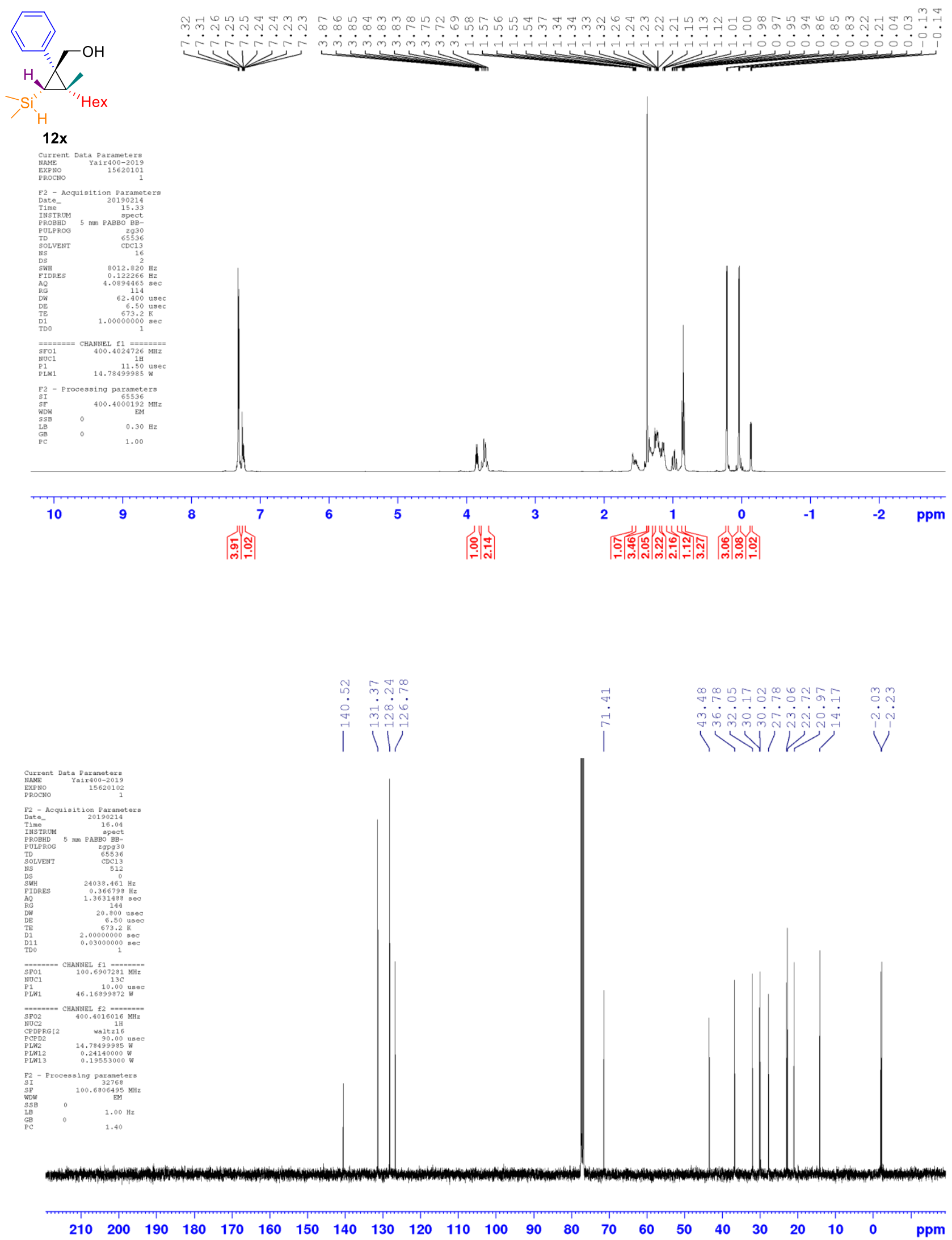


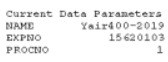

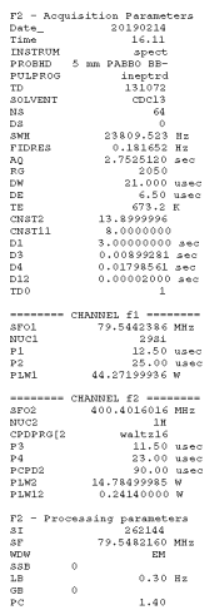

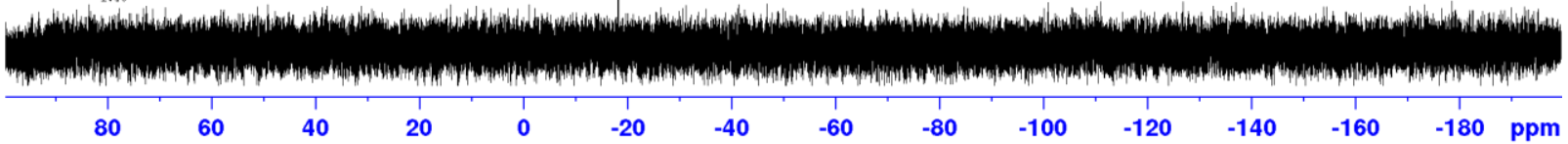



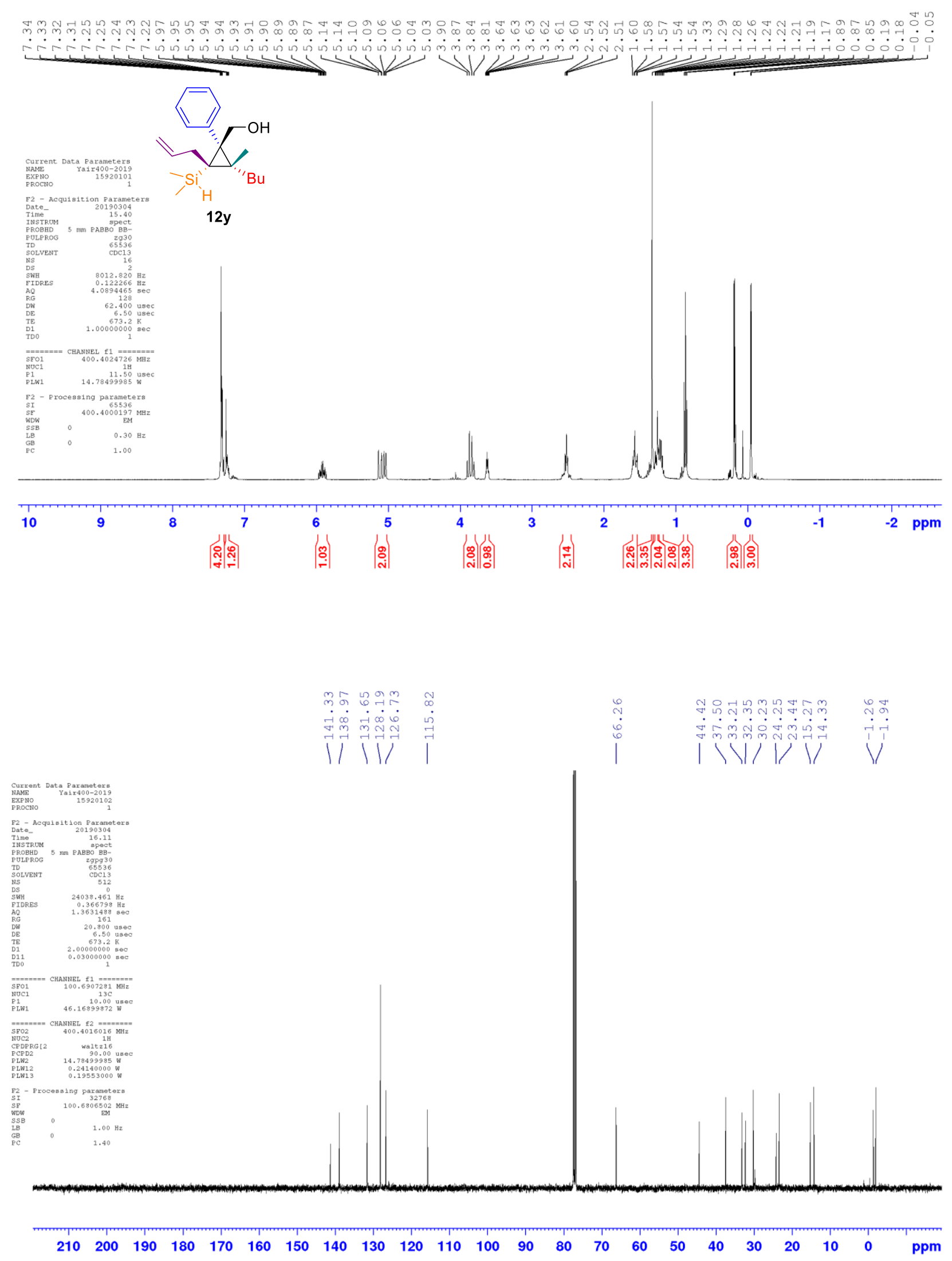


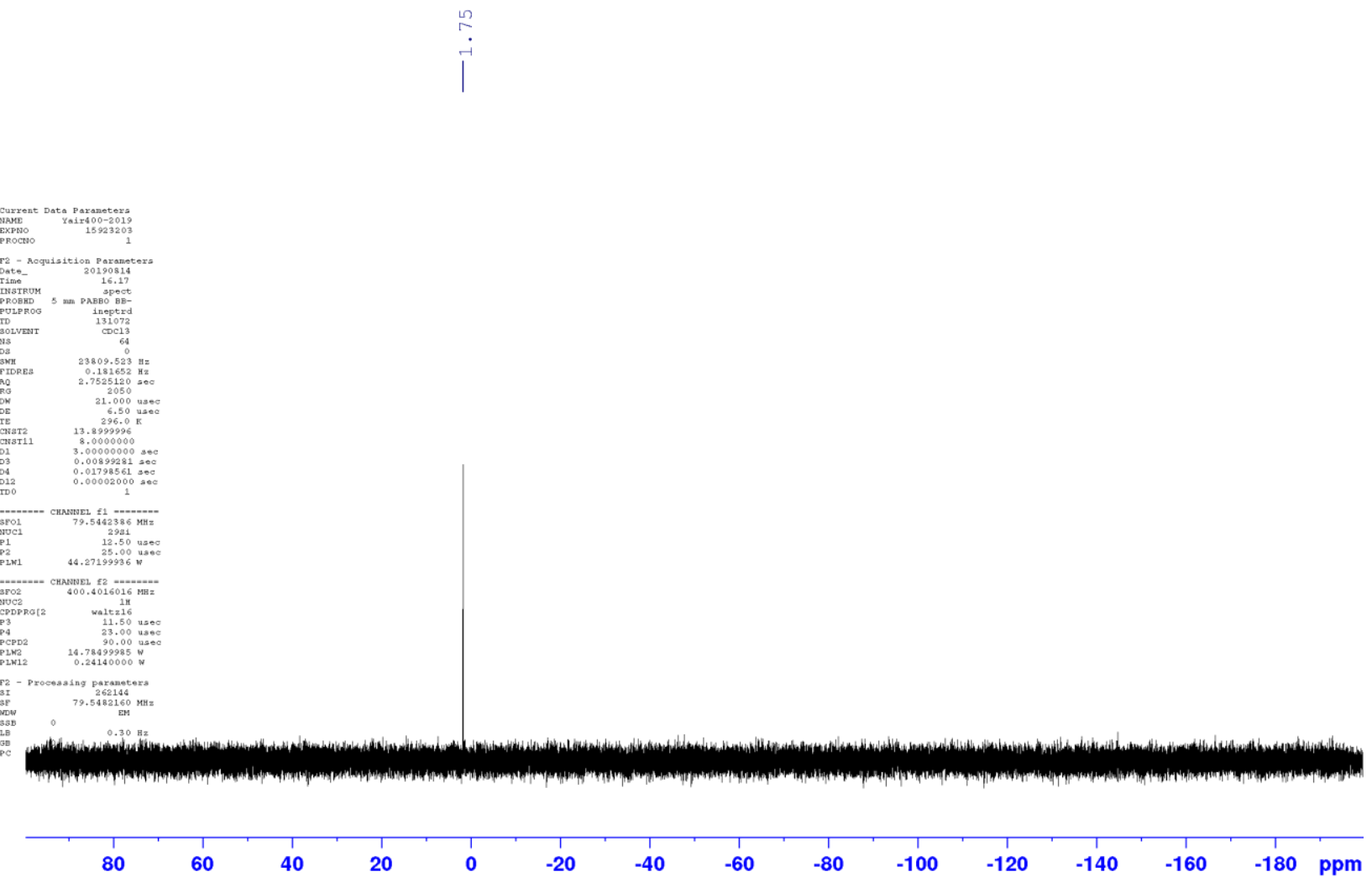



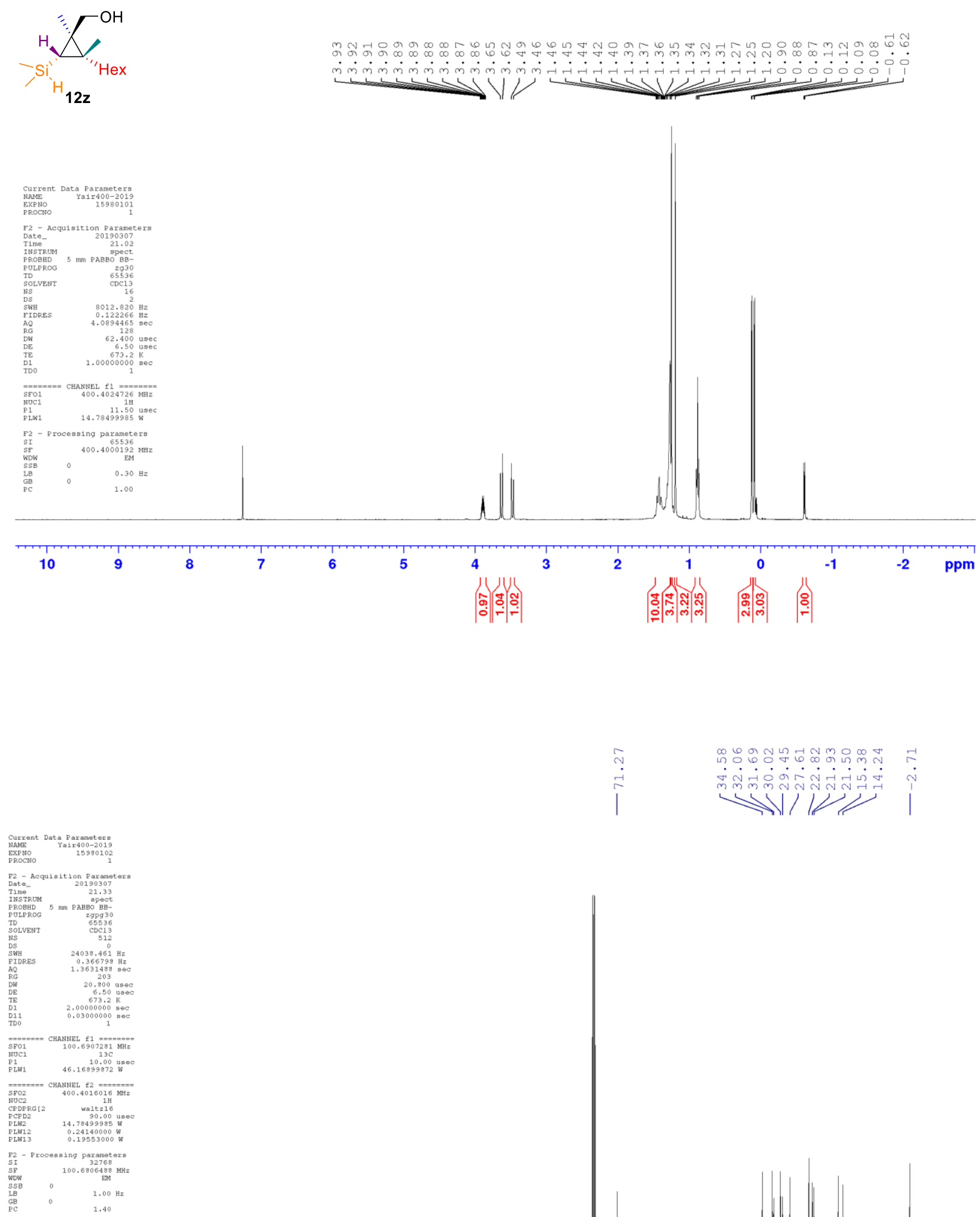


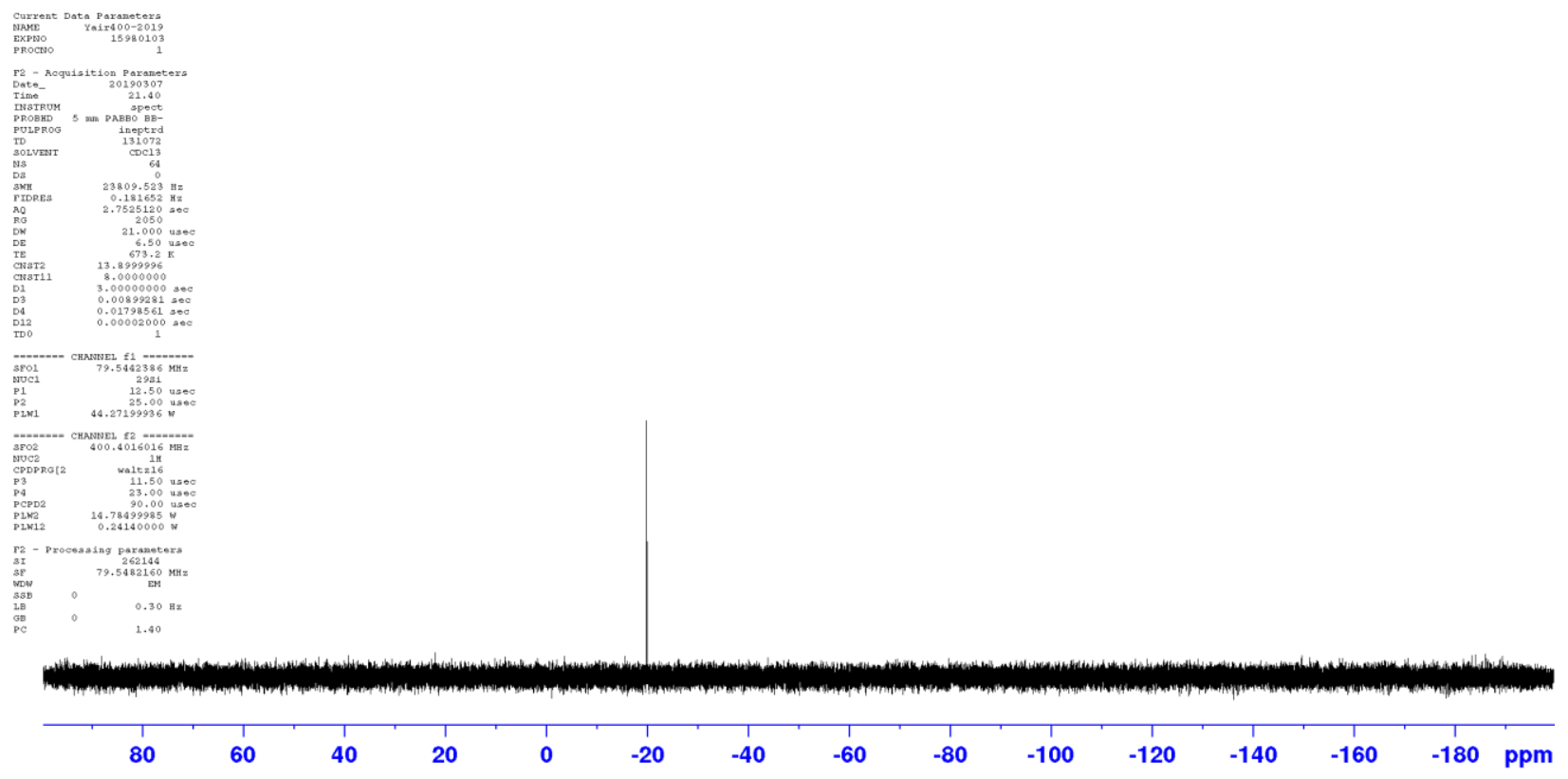


9. NMR spectra of cyclopropenylsilanes 11a, 11c-11h
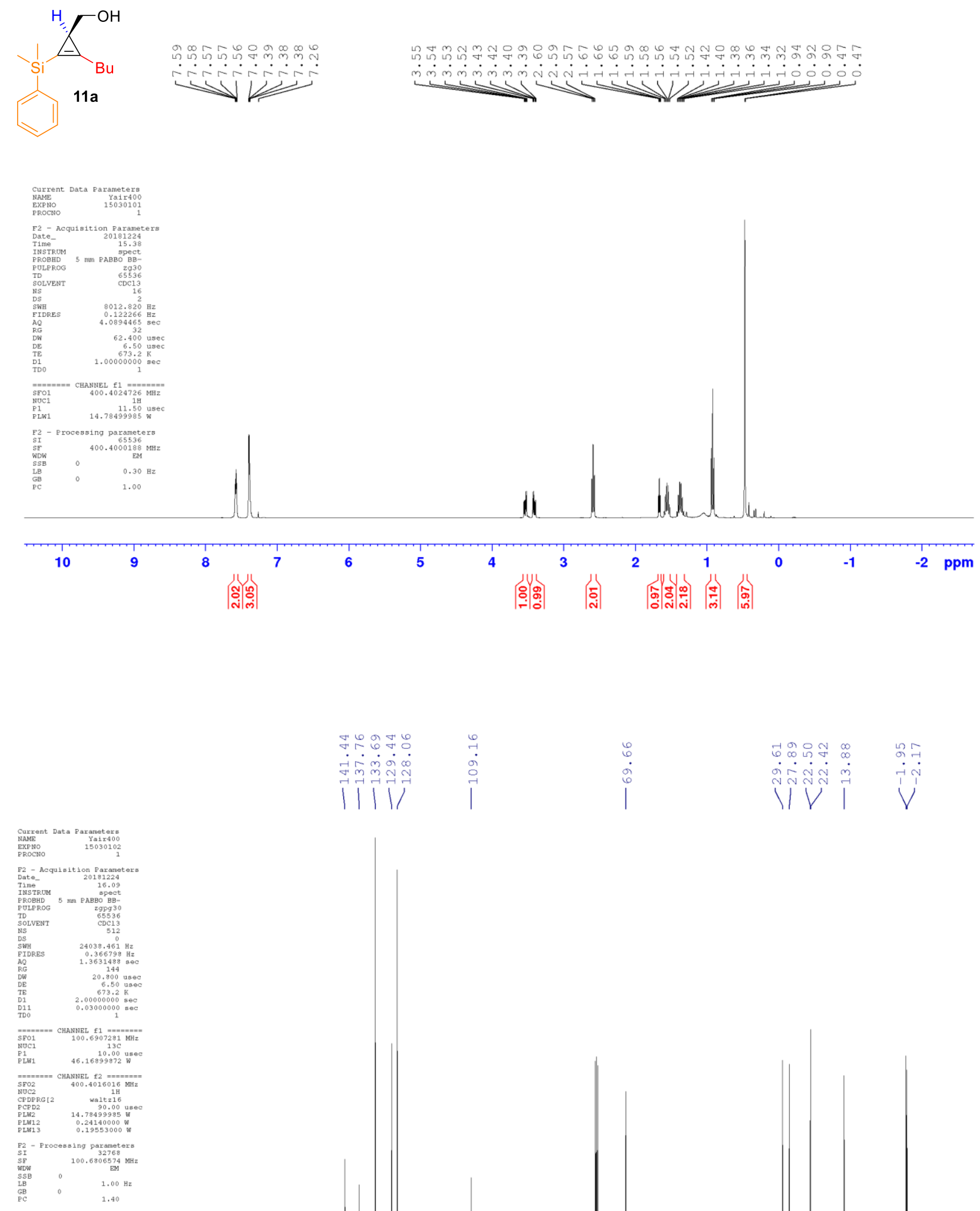


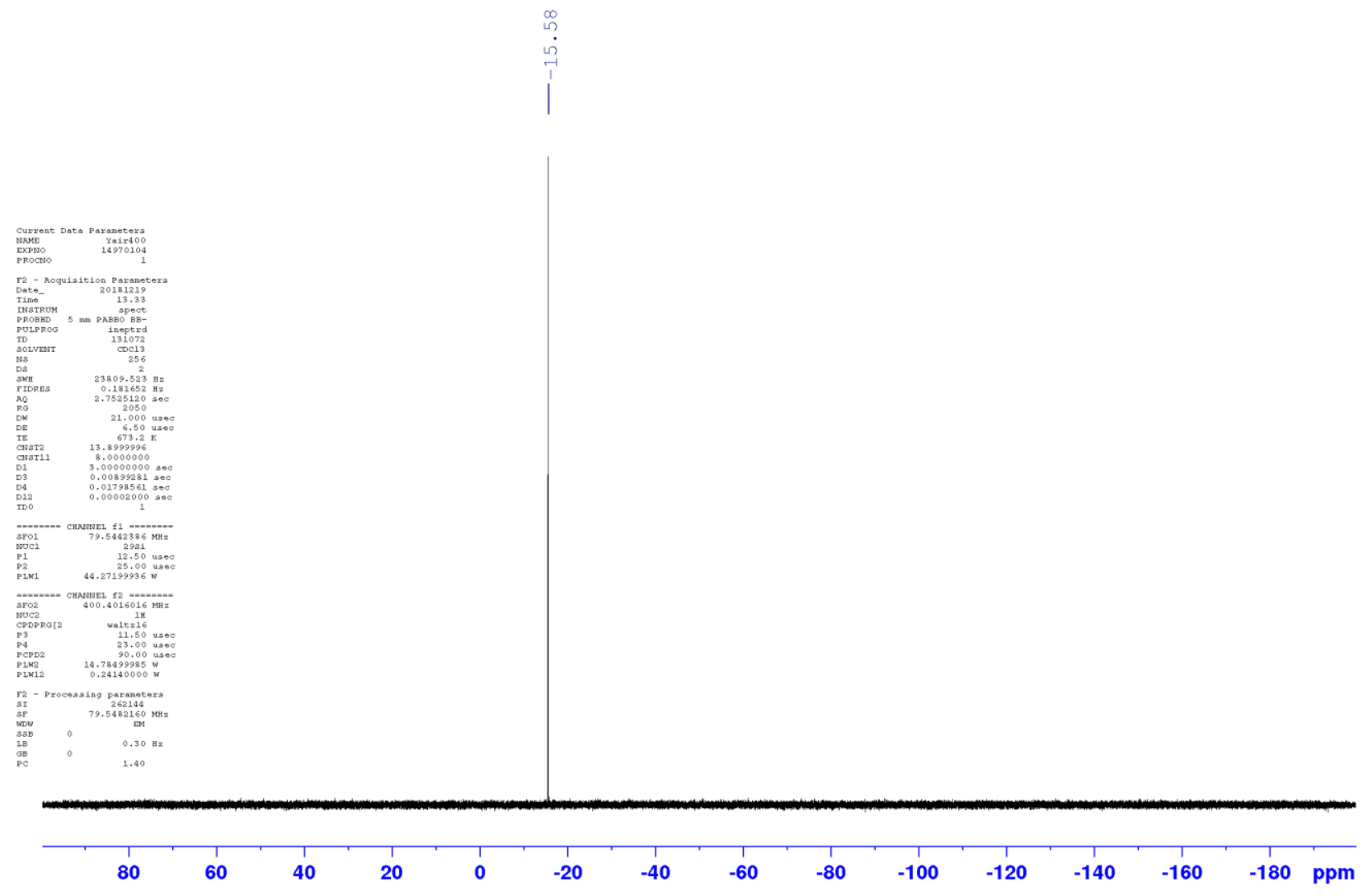



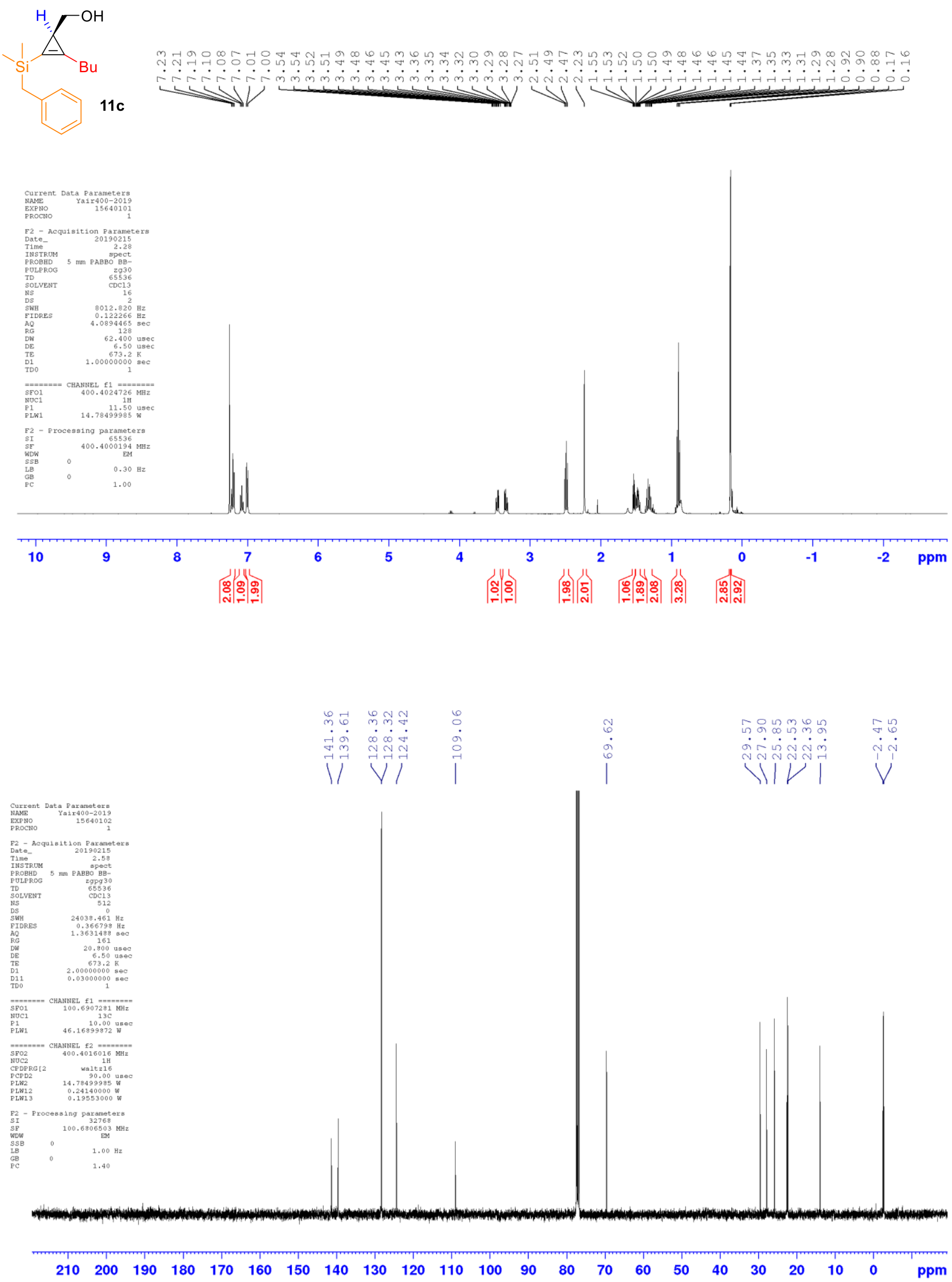


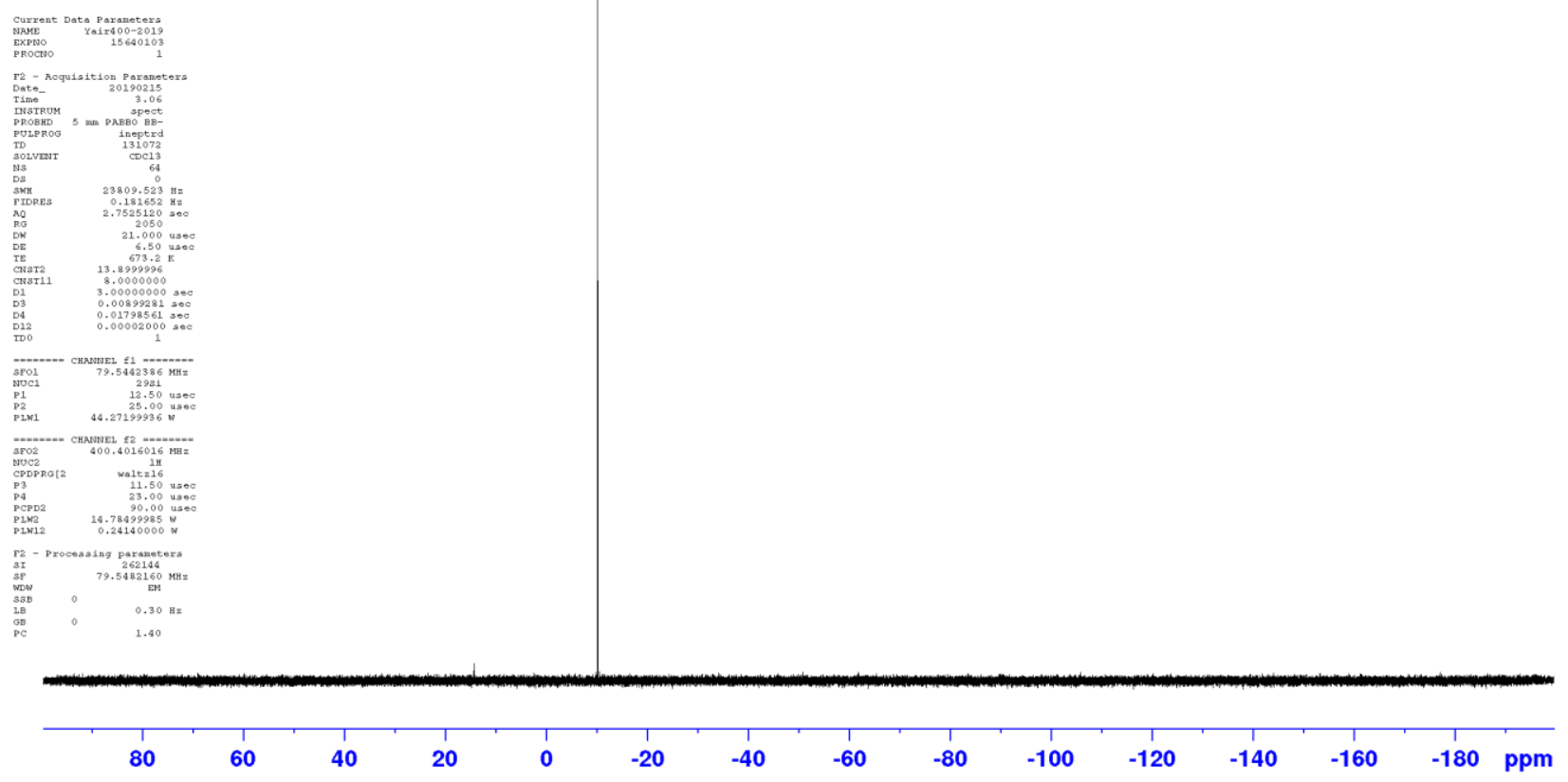



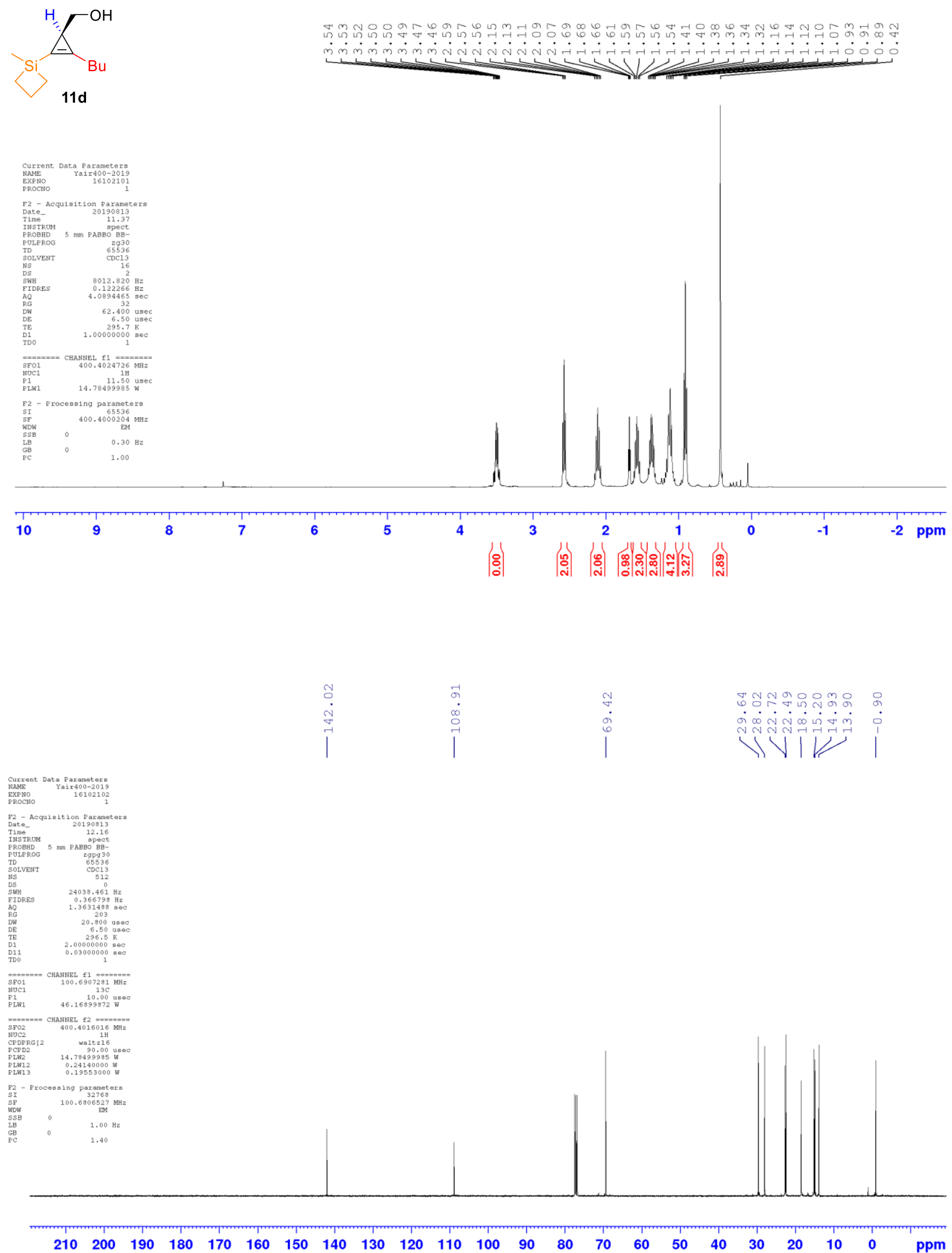


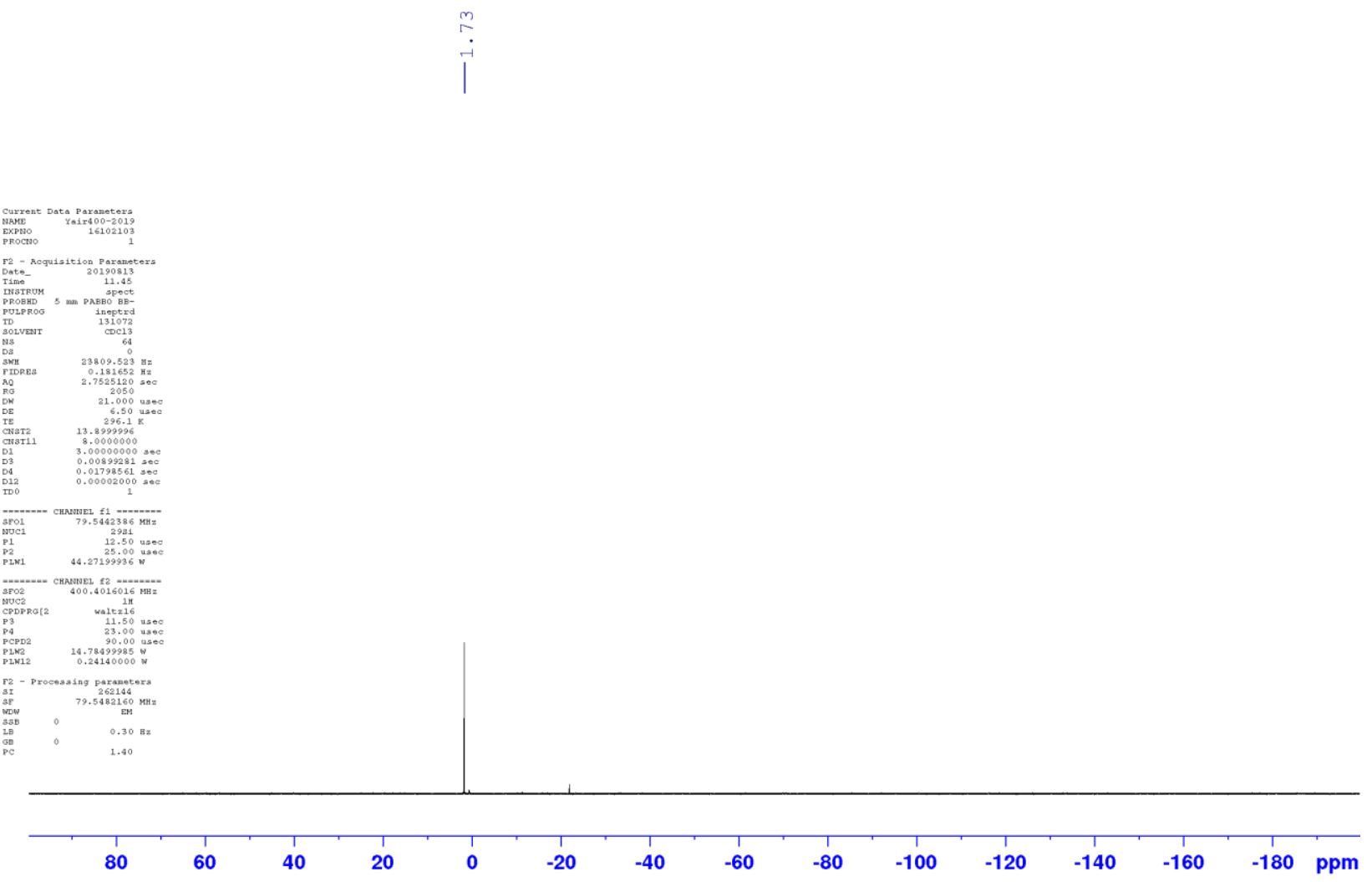



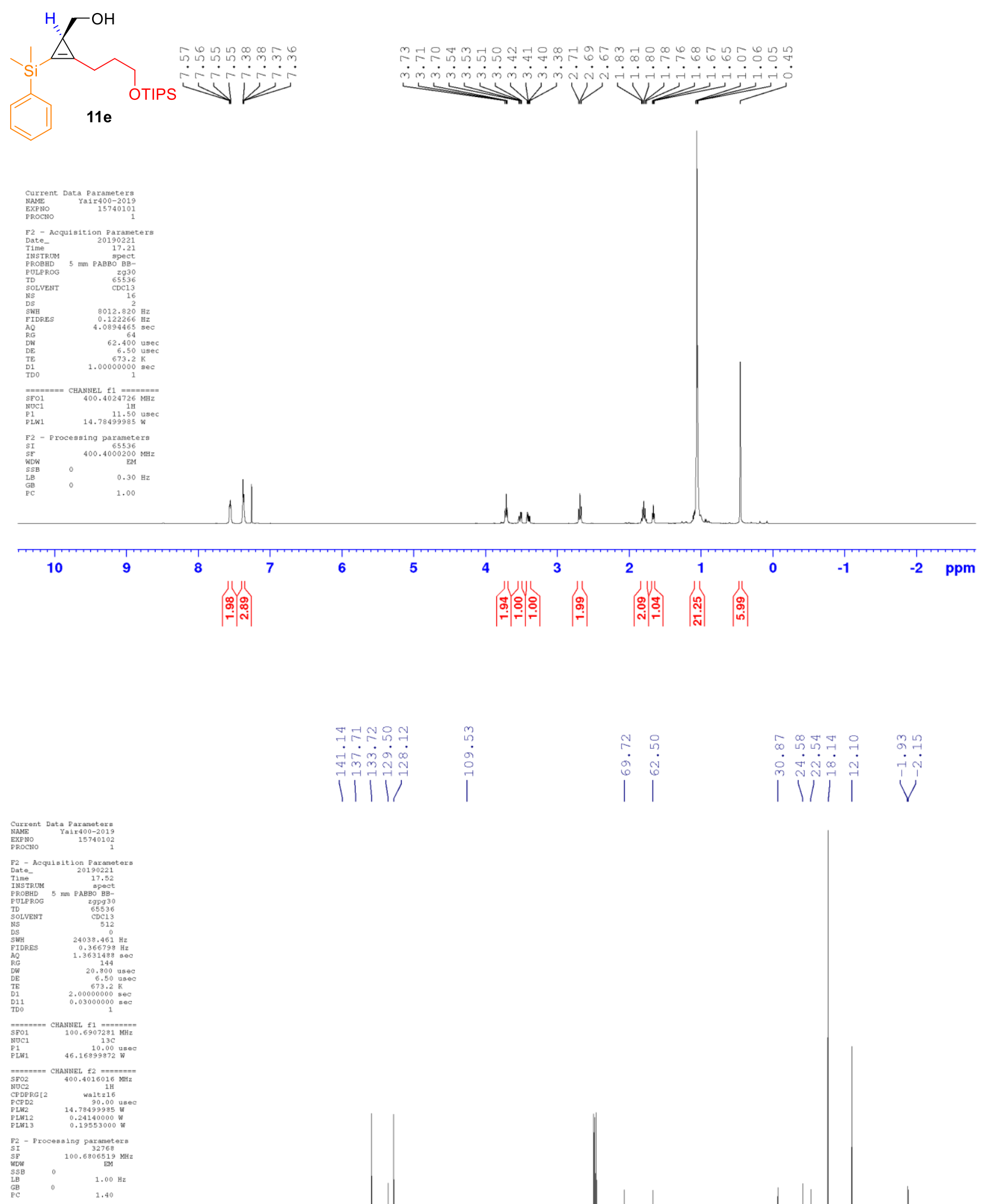


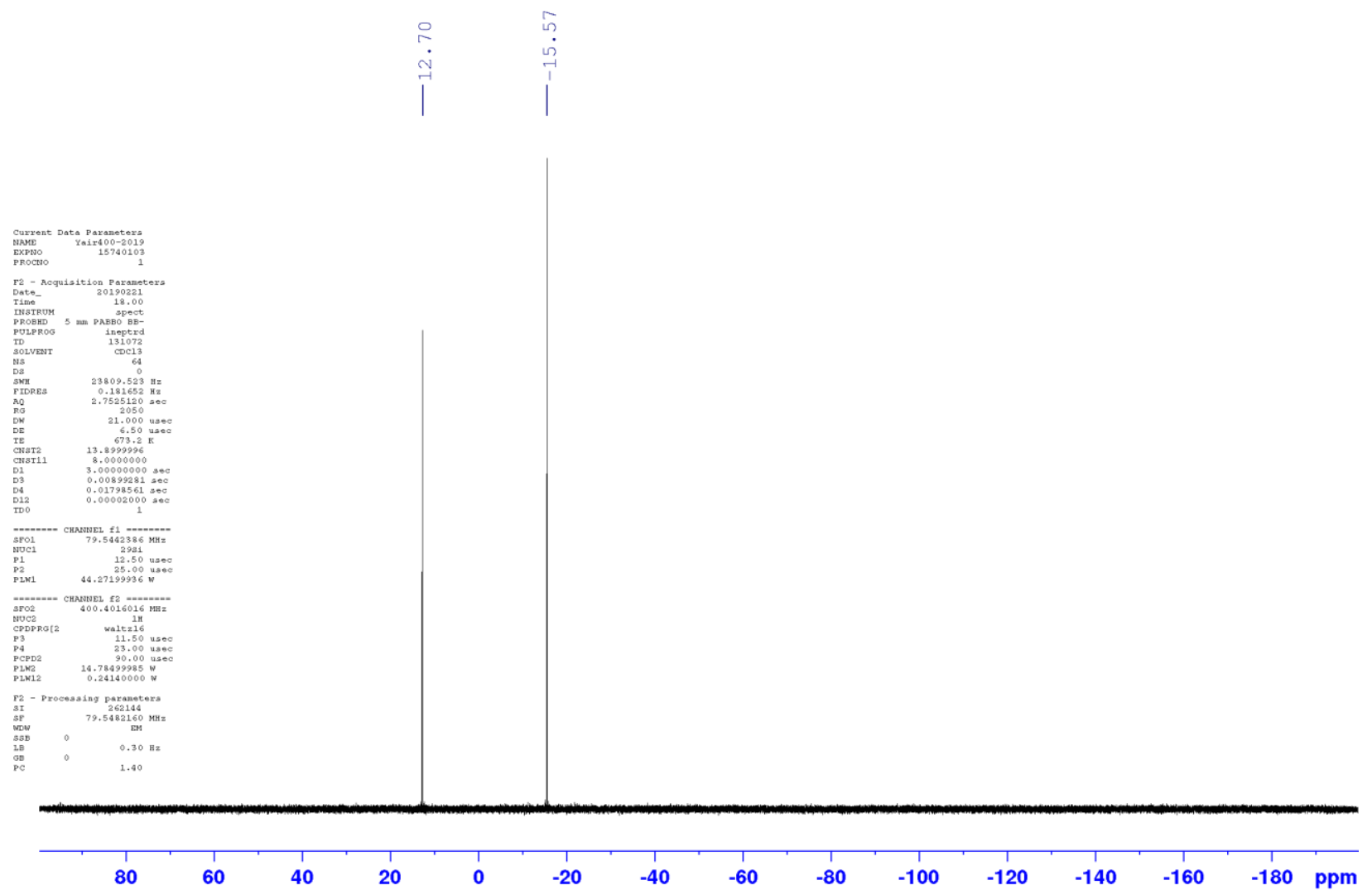




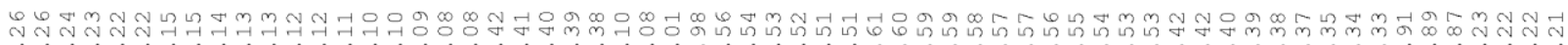

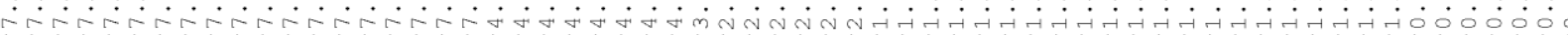

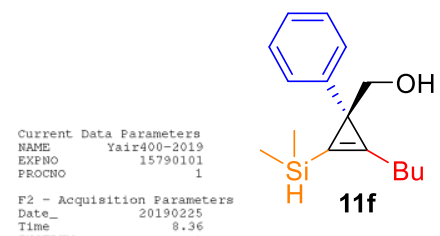

$\begin{array}{ll}\text { INSTRM } & 8.36 \\ \text { PROBHD } & \text { spect }\end{array}$

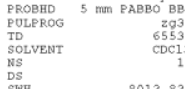

$\begin{array}{lr}\text { DS } & 16 \\ \text { SWH } & 8012.820 \mathrm{~Hz} \\ \text { FIDDES } & 0.122266 \mathrm{~Hz} \\ \text { AO } & 4.089465 \mathrm{He} \\ \text { RG } & 90.5 \\ \text { De } & 69.400\end{array}$

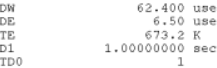

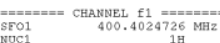

11.50 use
P1
PL1
PL1

F2 - processing parameters
SI

$\begin{array}{ll}\text { SI } & 65536 \\ \text { SE } & 400.4000341 \\ \text { WOW } & \text { FM }\end{array}$

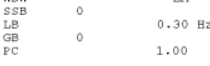

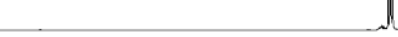

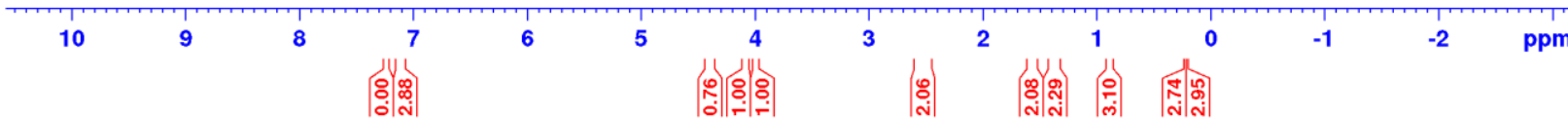
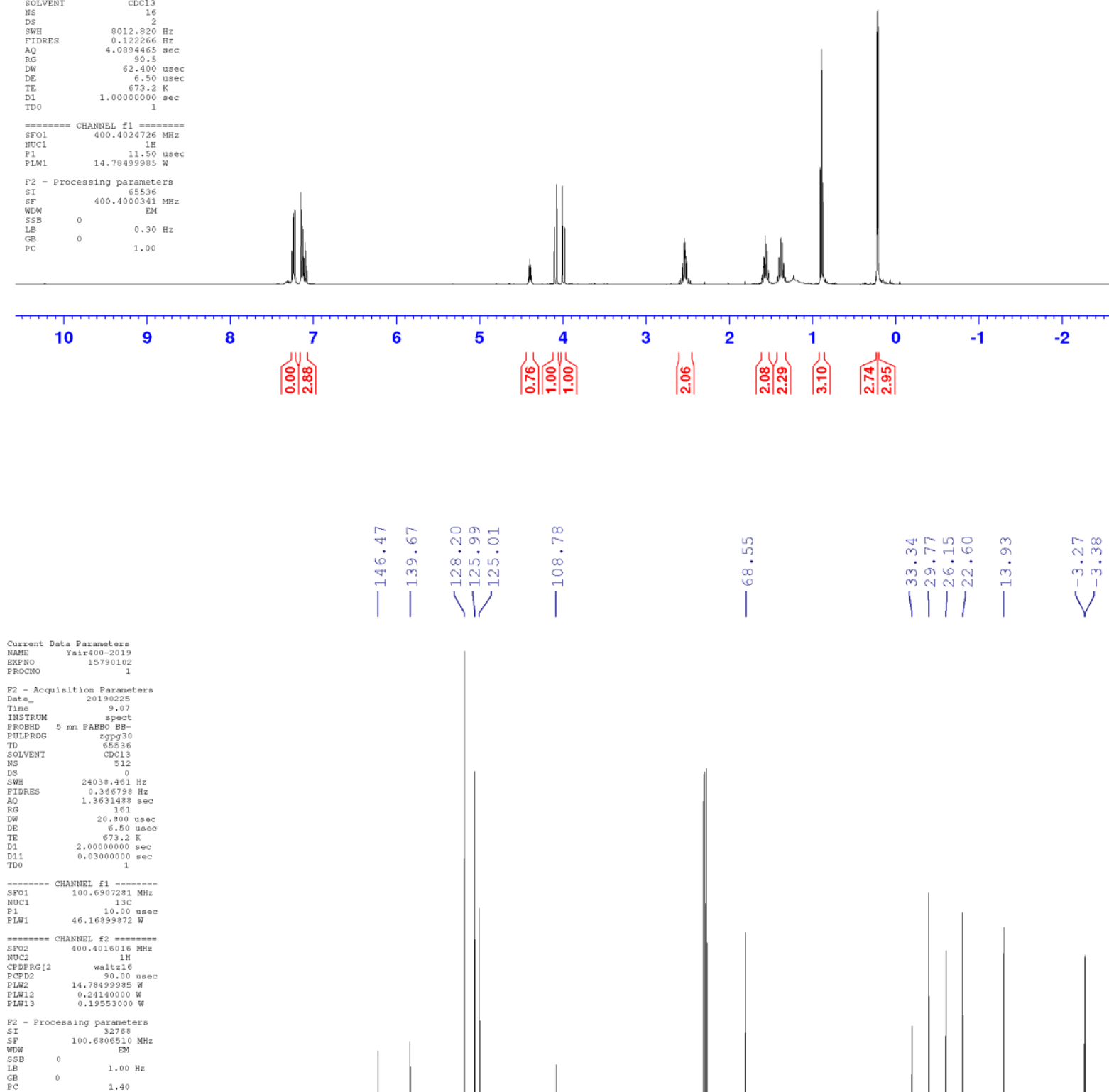

pm 


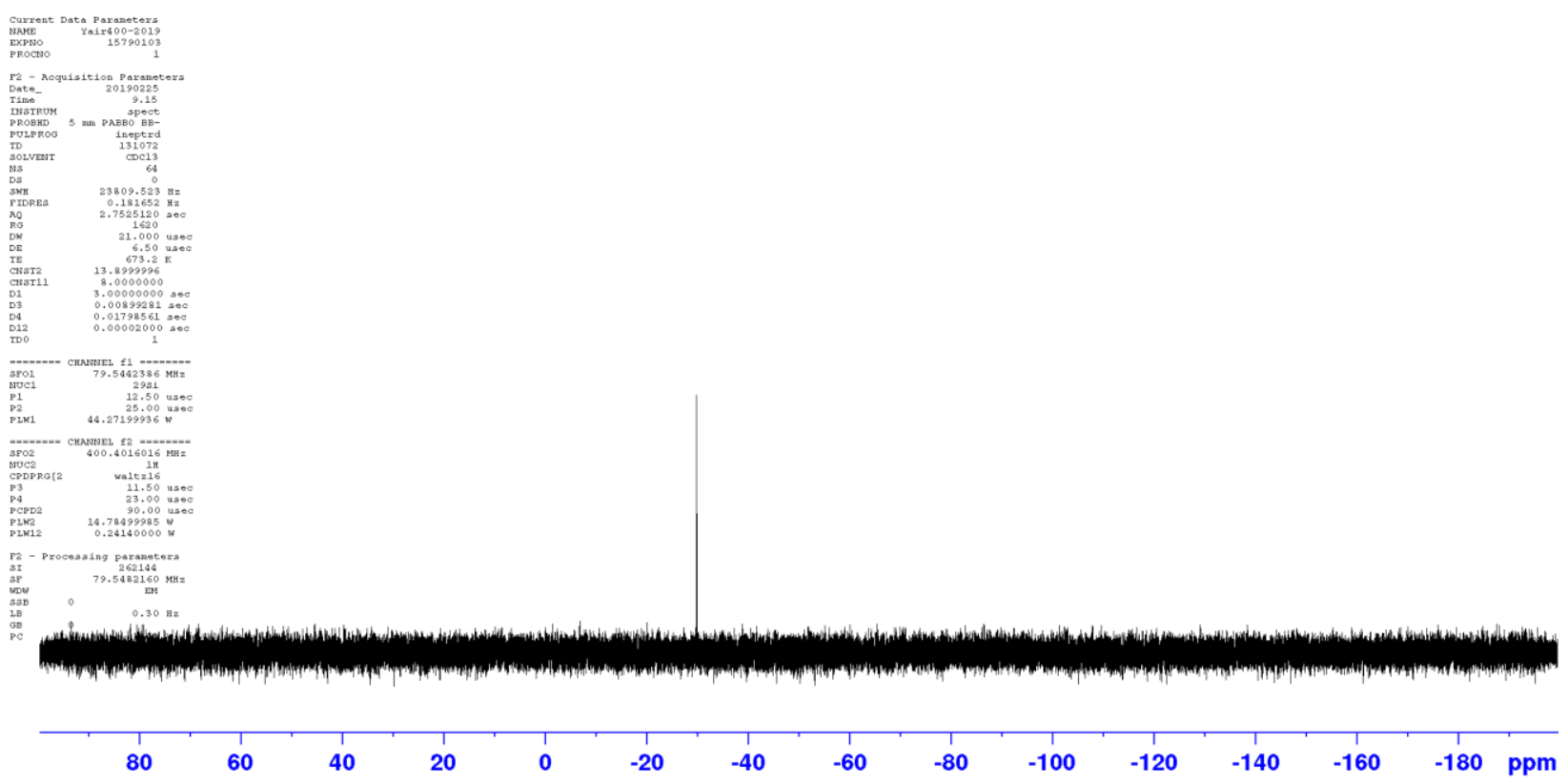




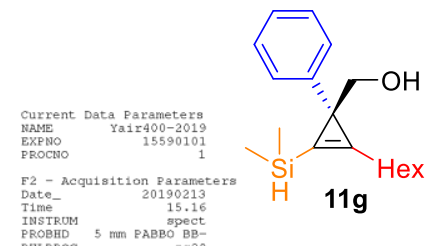

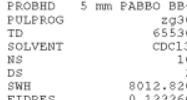

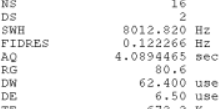

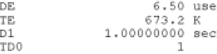

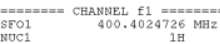

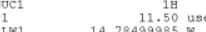

F2 - Processing parameters

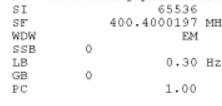
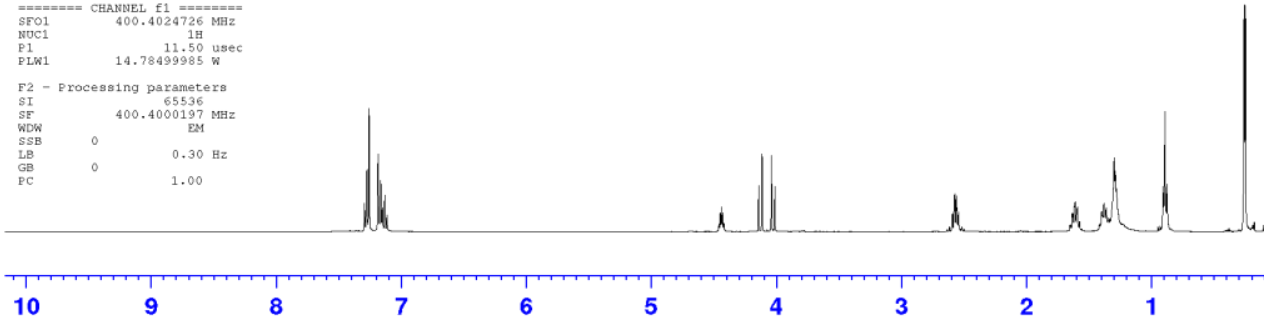

8

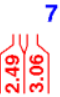
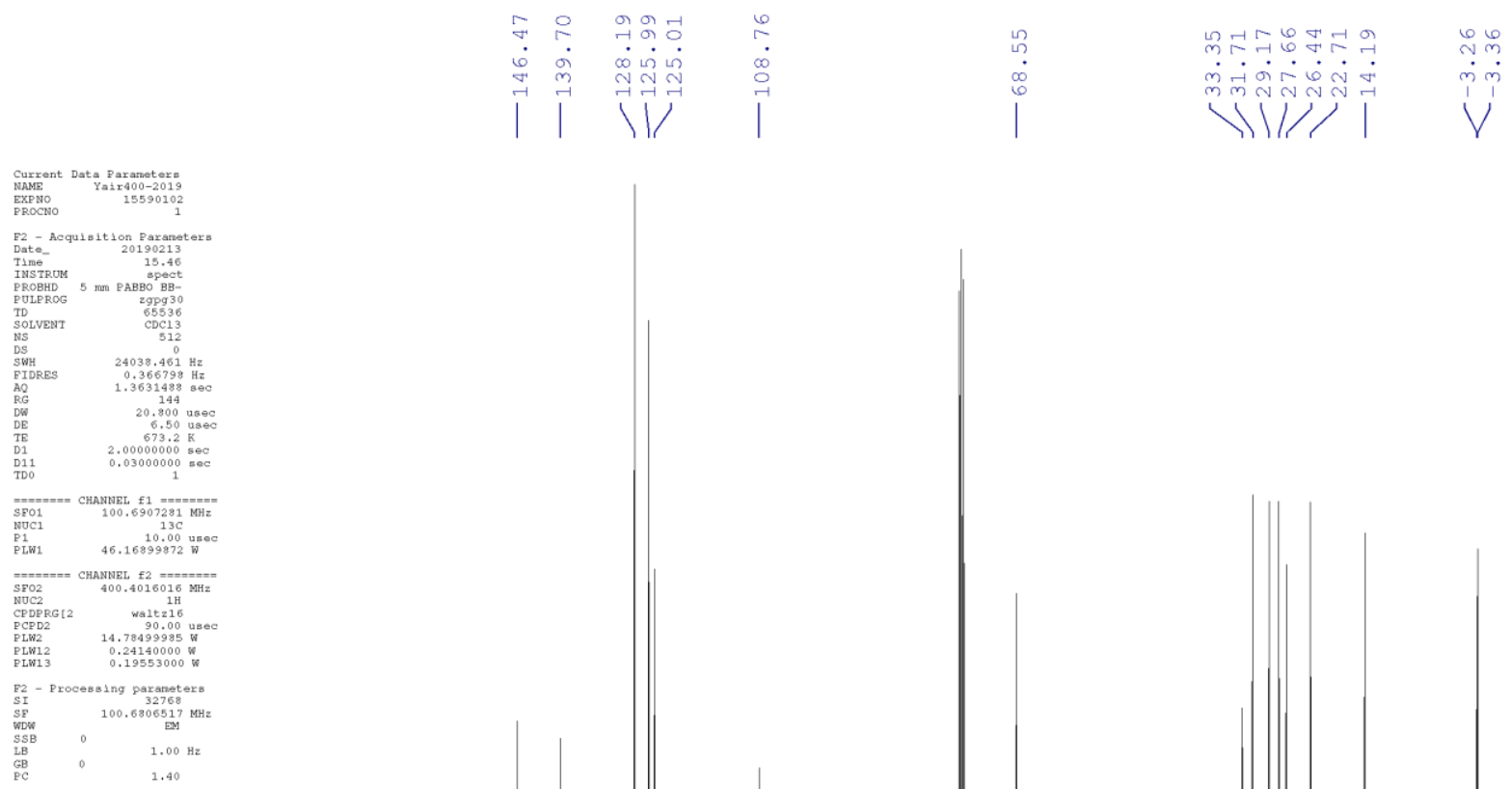

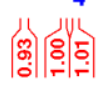

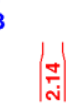

2

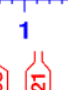

0
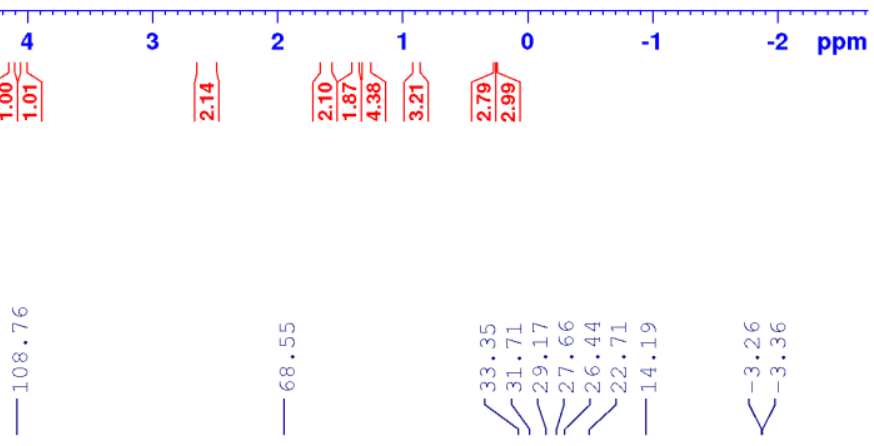

$\begin{array}{ll}6 & 6 \\ ن & m \\ \dot{n} & \dot{m} \\ v\end{array}$
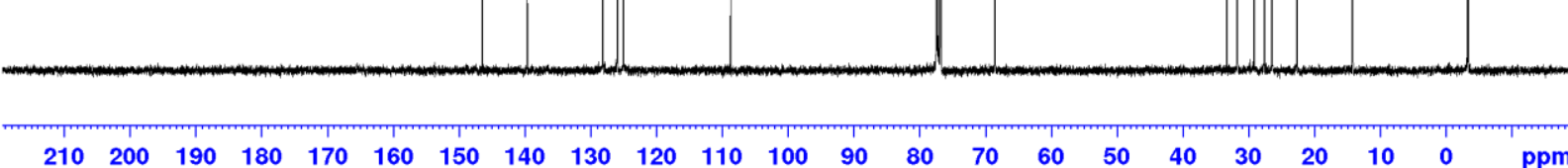


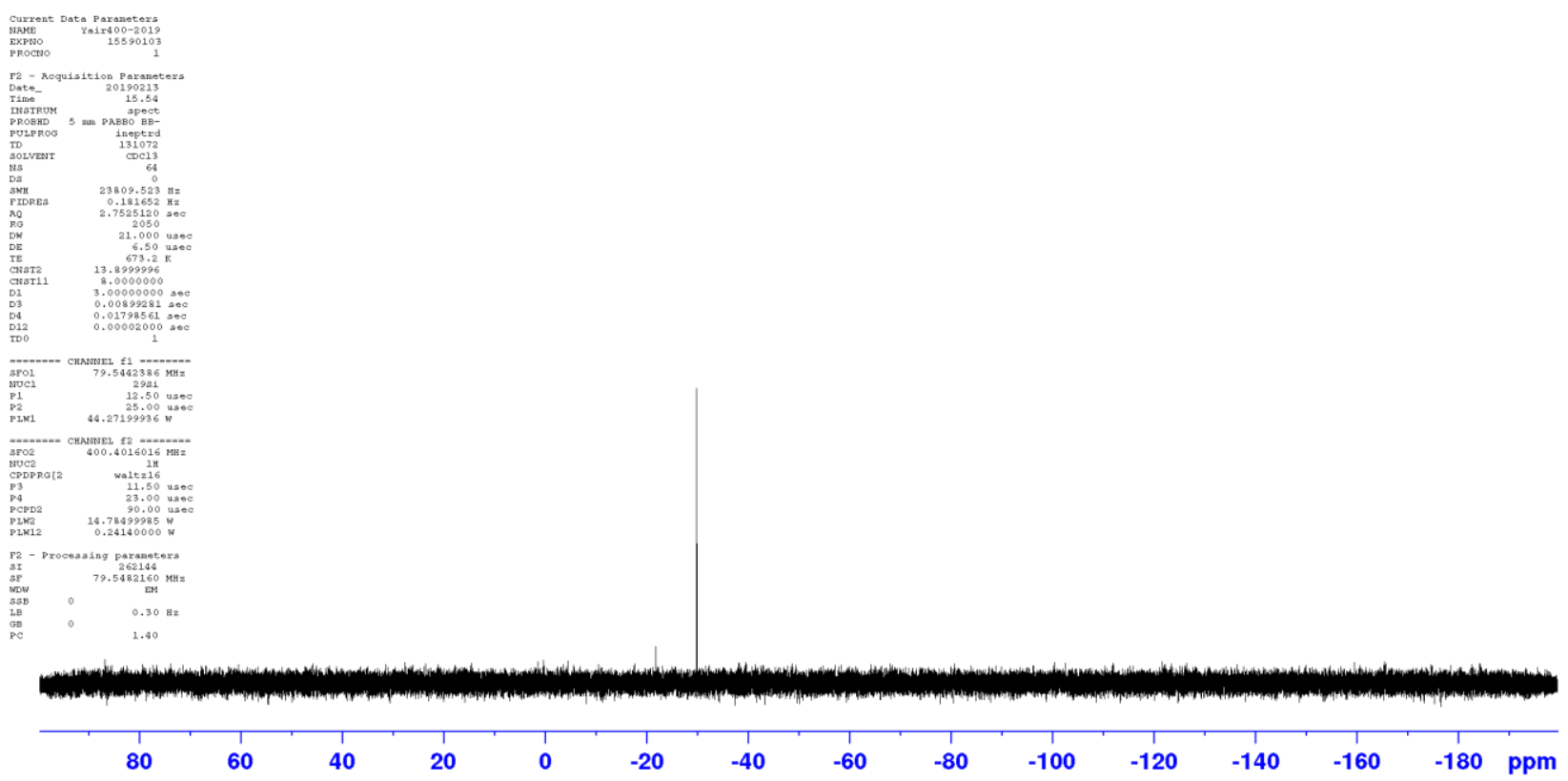



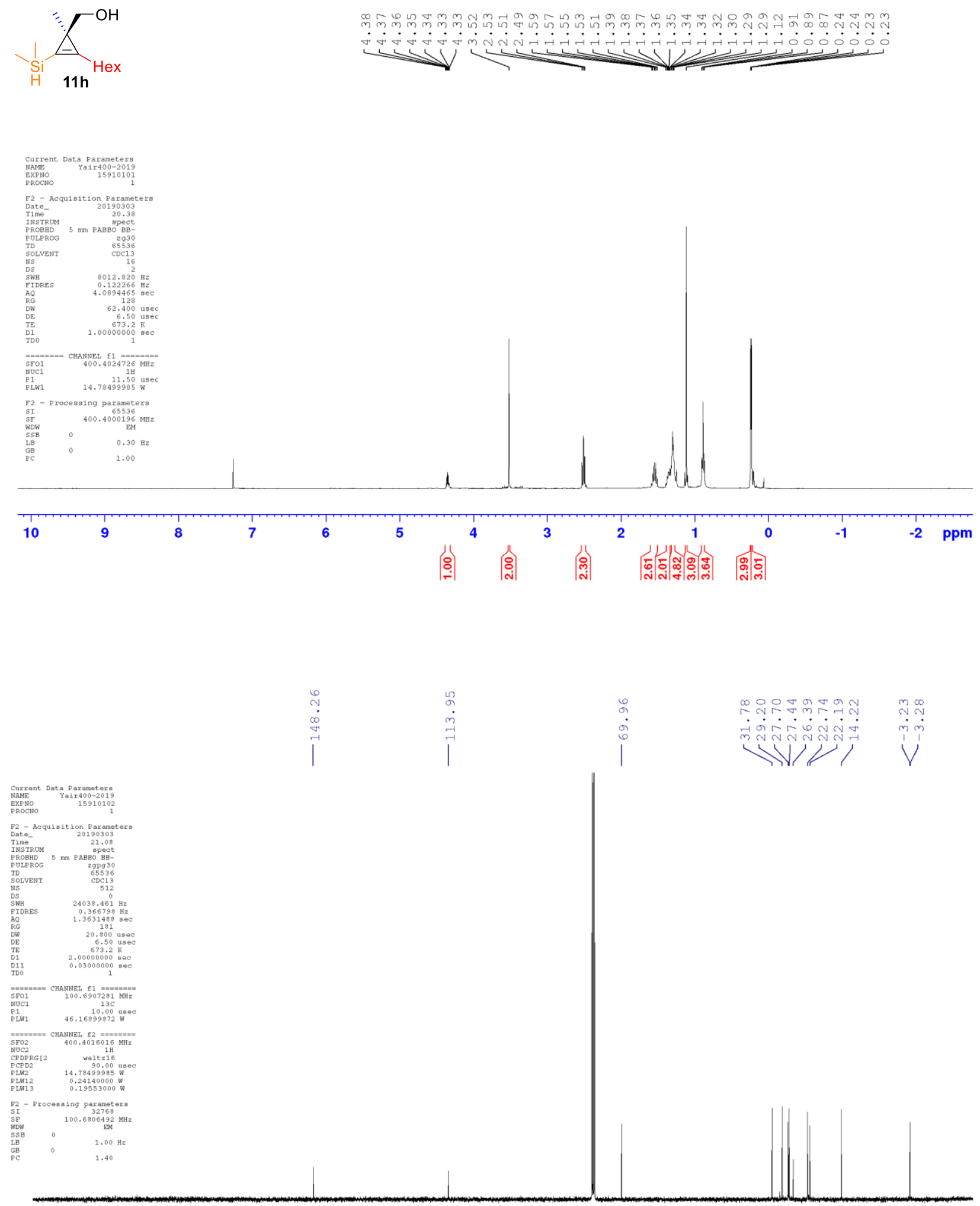

$\begin{array}{llllllllllllllllllllllllll}210 & 200 & 190 & 180 & 170 & 160 & 150 & 140 & 130 & 120 & 110 & 100 & 90 & 80 & 70 & 60 & 50 & 40 & 30 & 20 & 10 & 0 & \text { ppm }\end{array}$ 


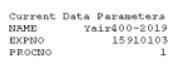

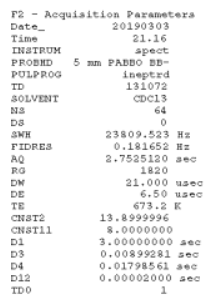

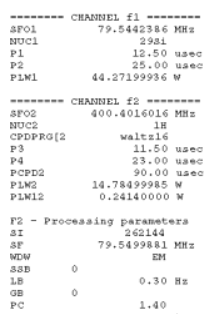

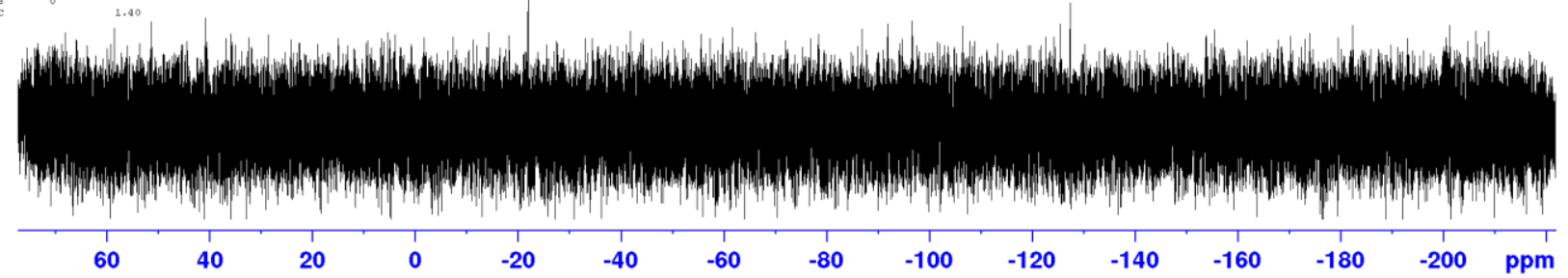


10. NMR spectra of cyclopropenylsilanes $\mathbf{1 3 a}, \mathbf{1 3 c - 1 3 h}$.
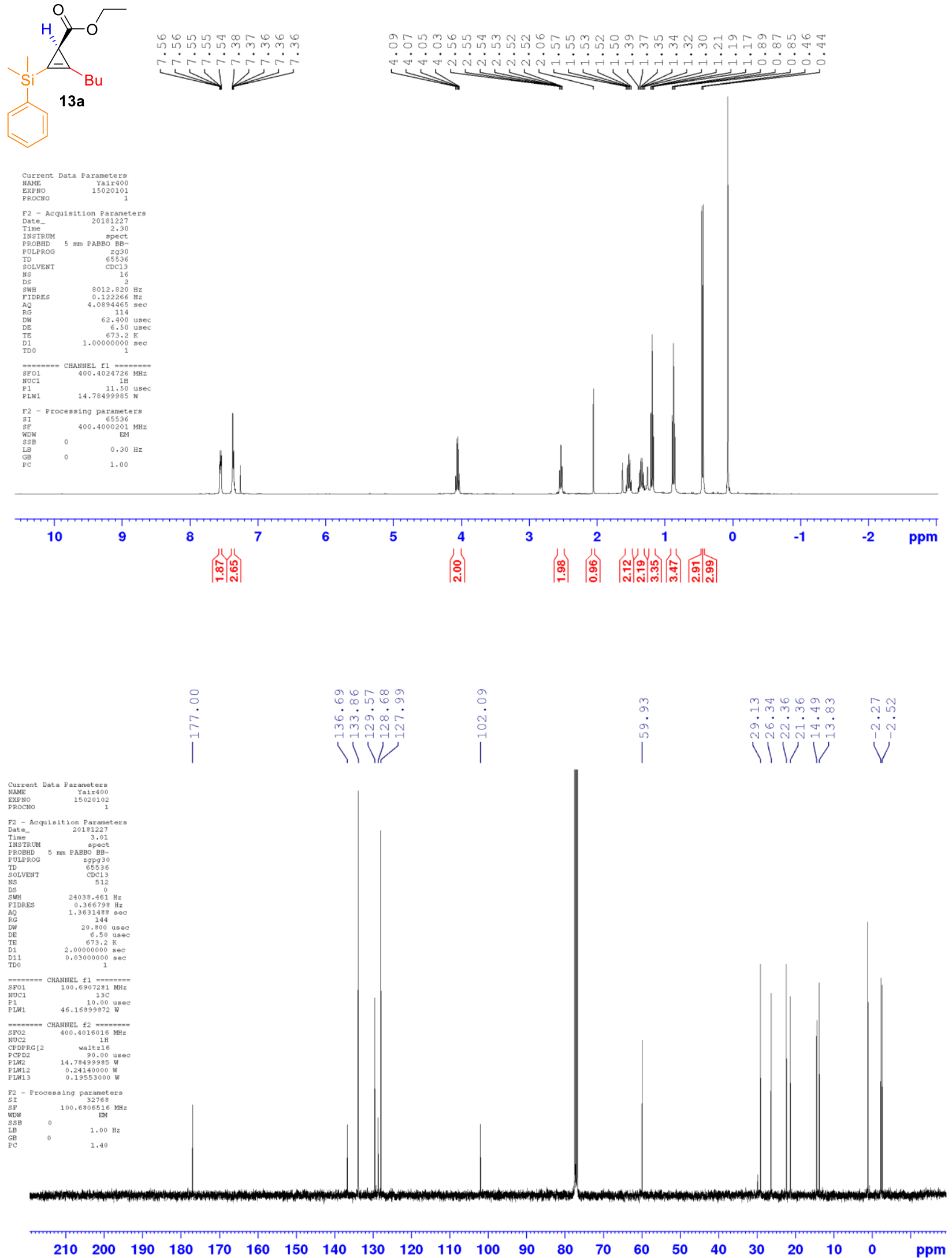

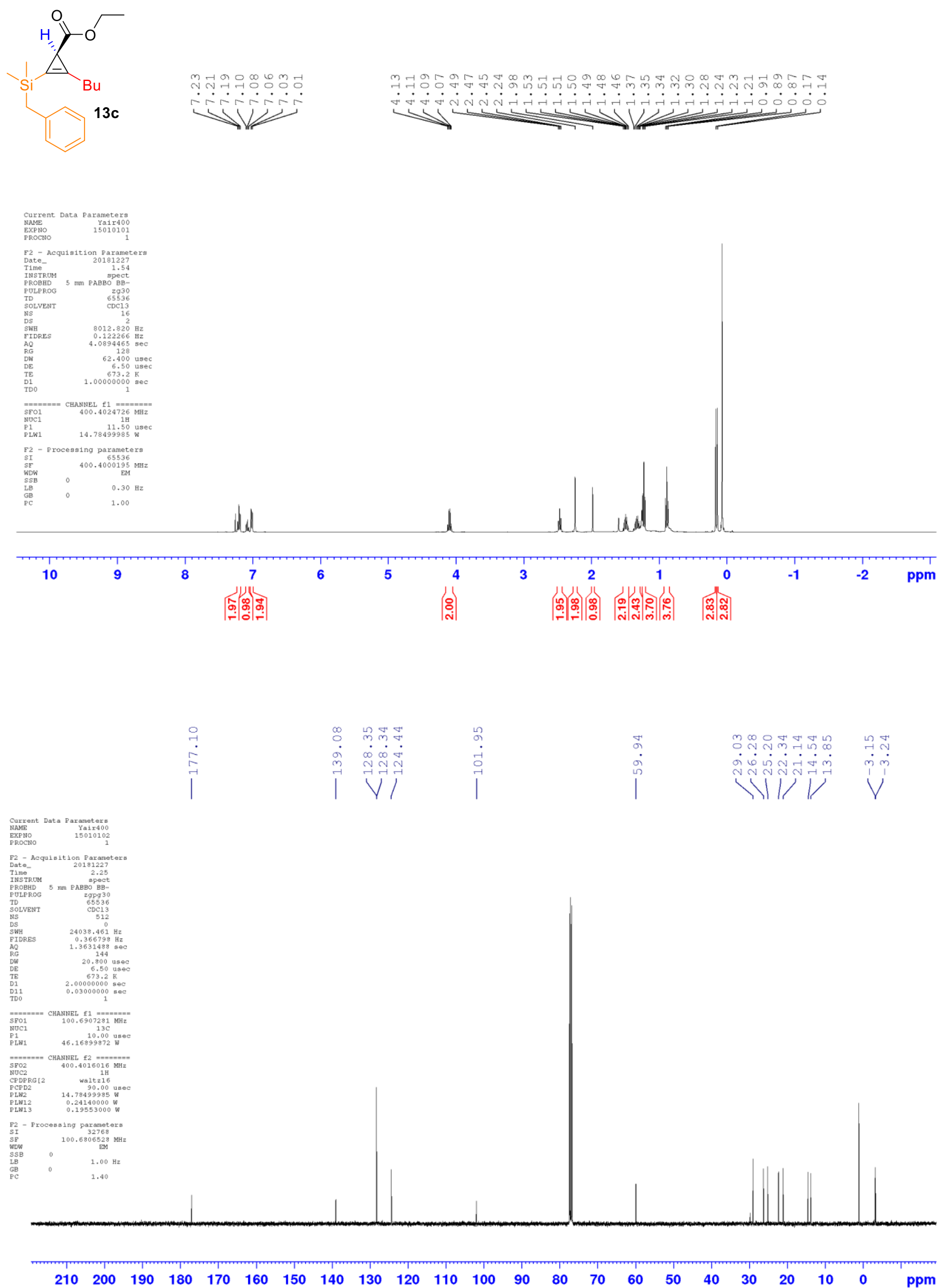

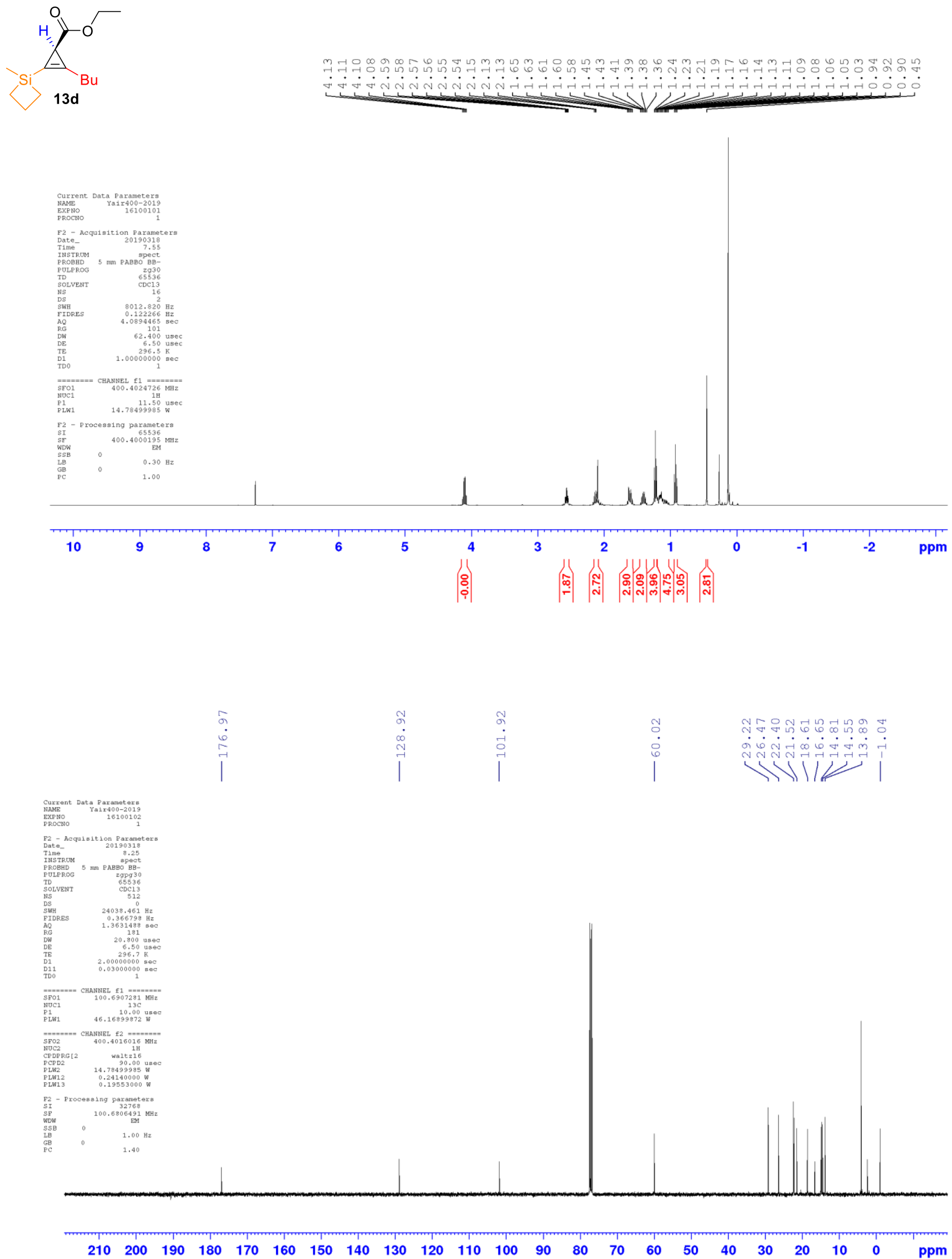

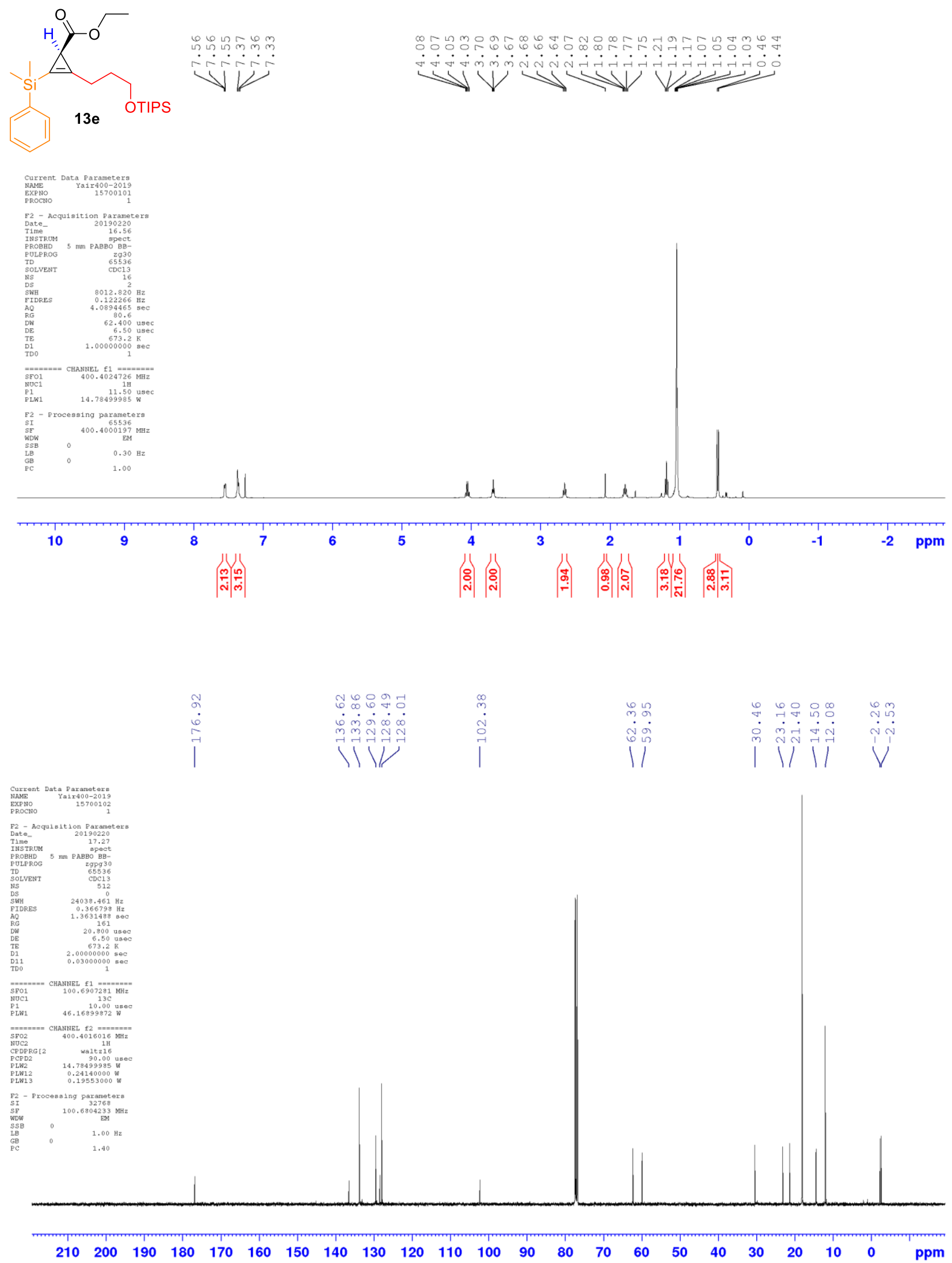

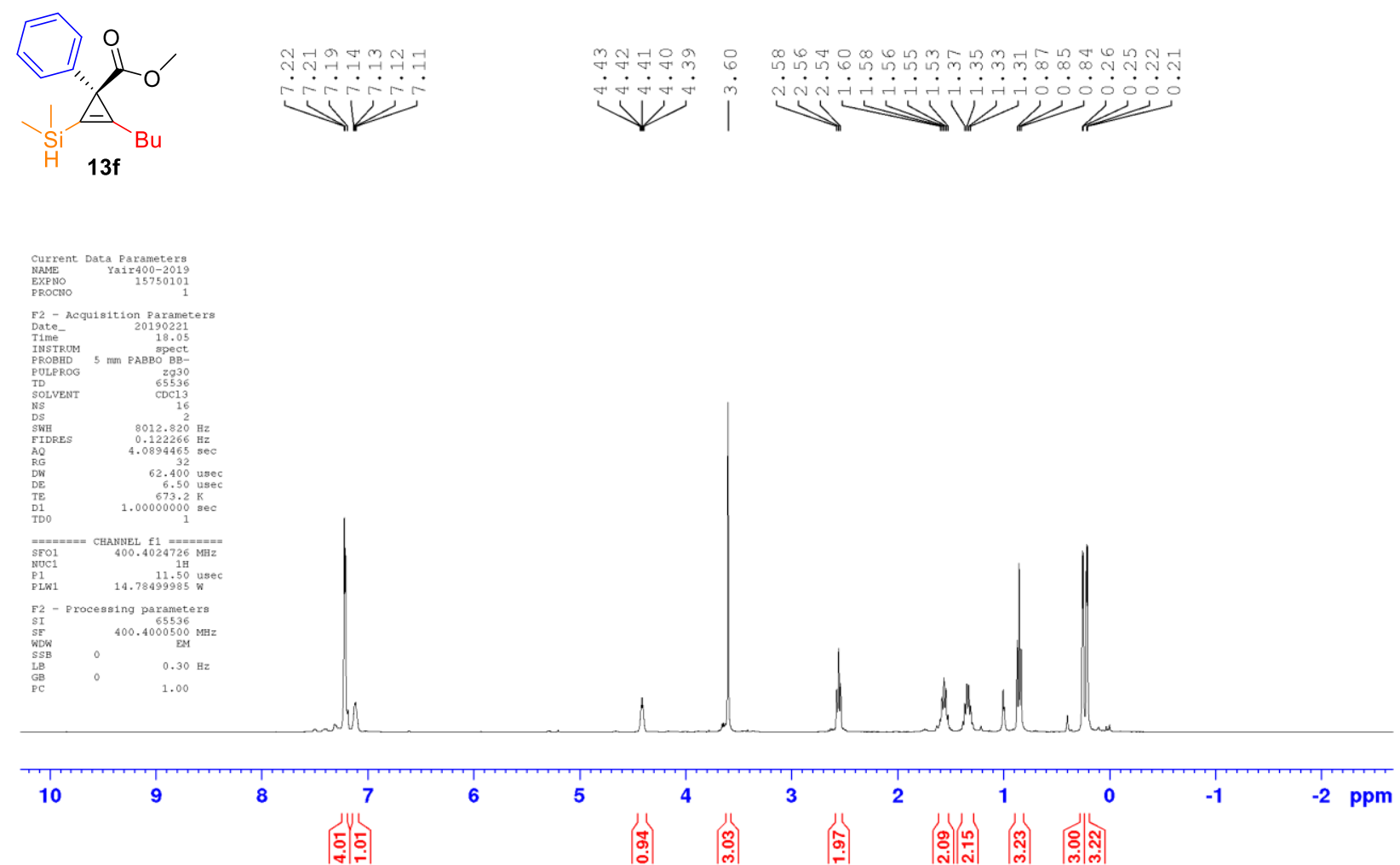

|하함

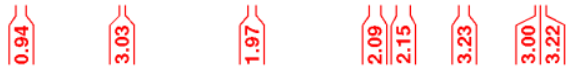
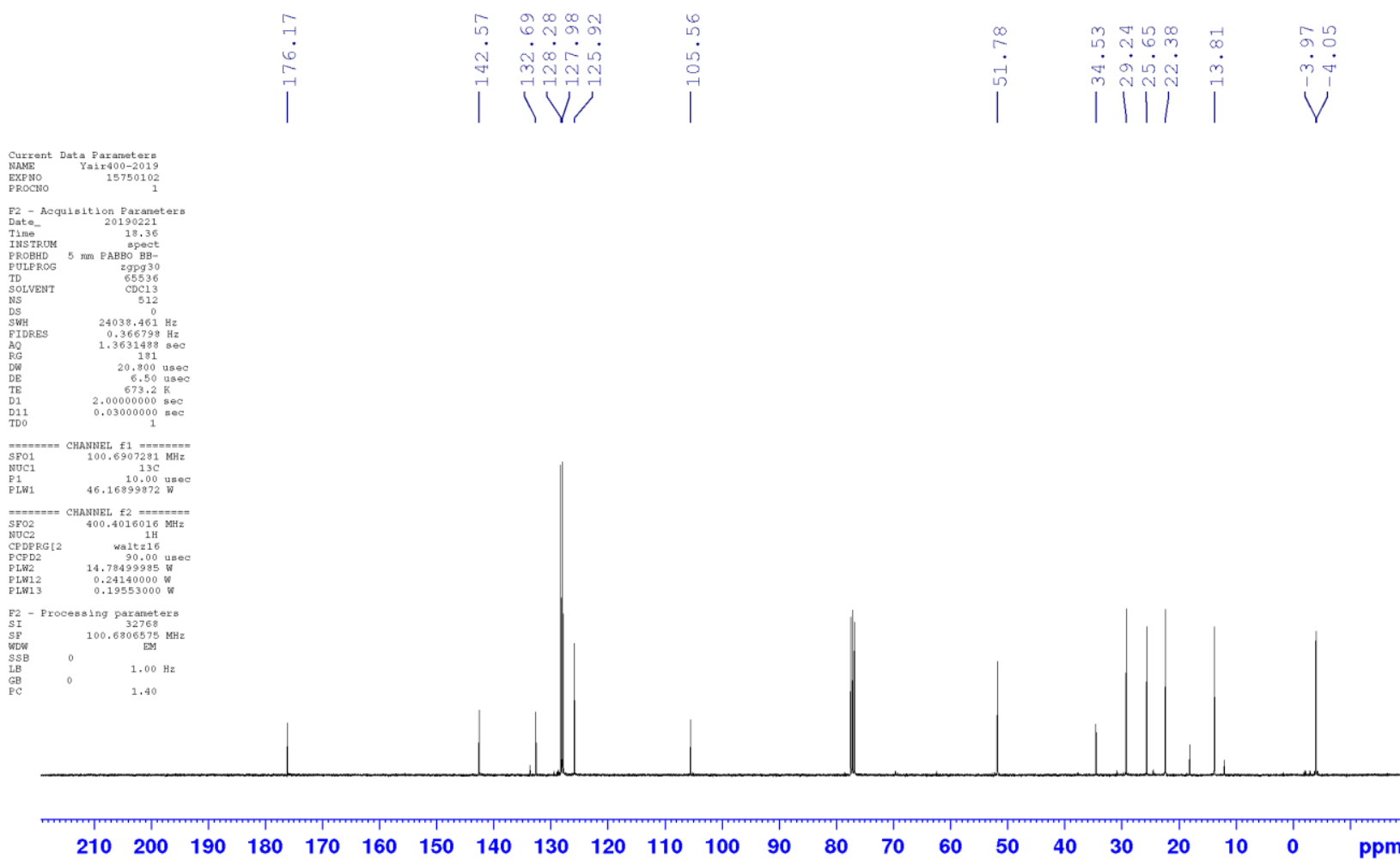

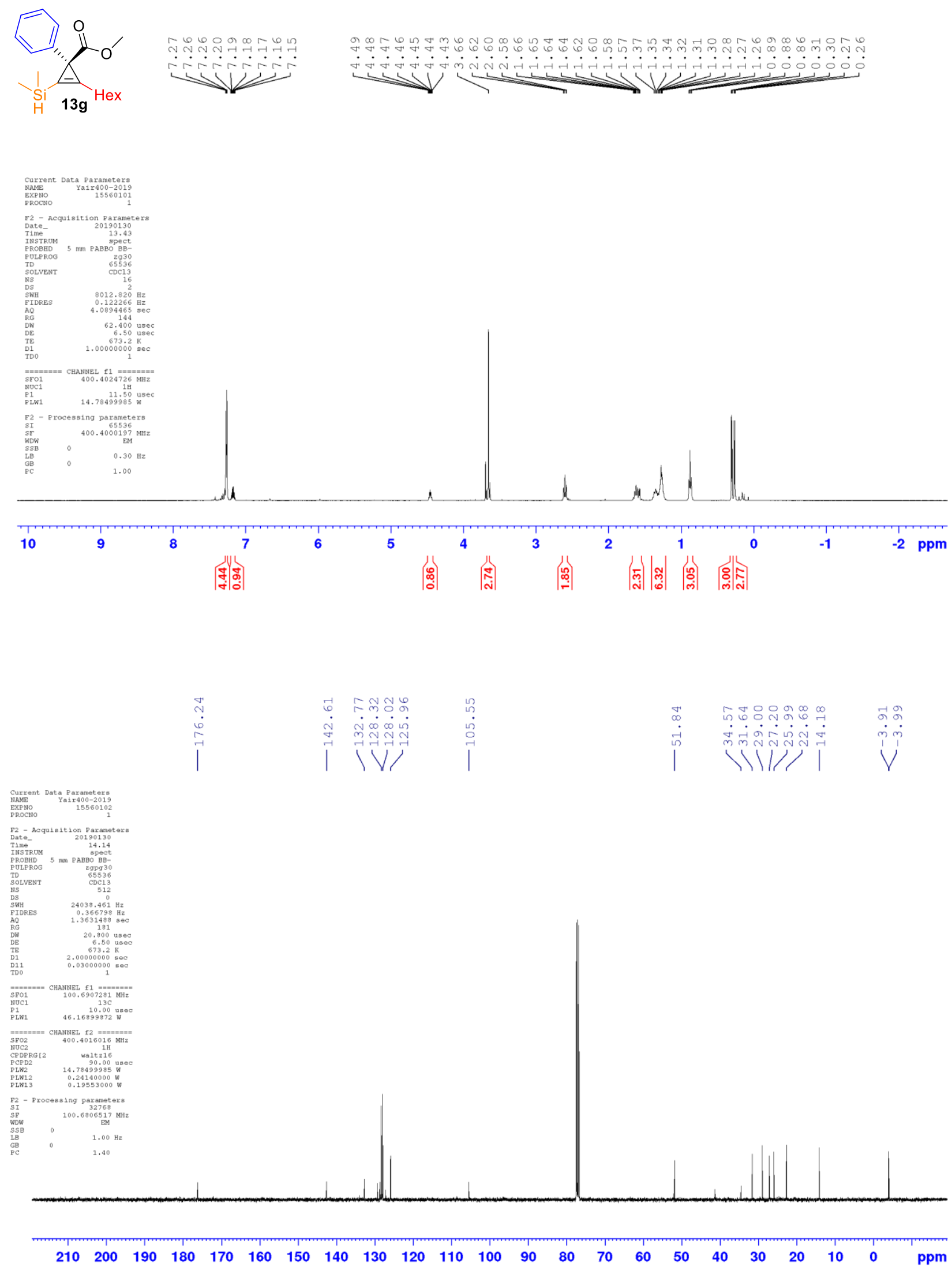

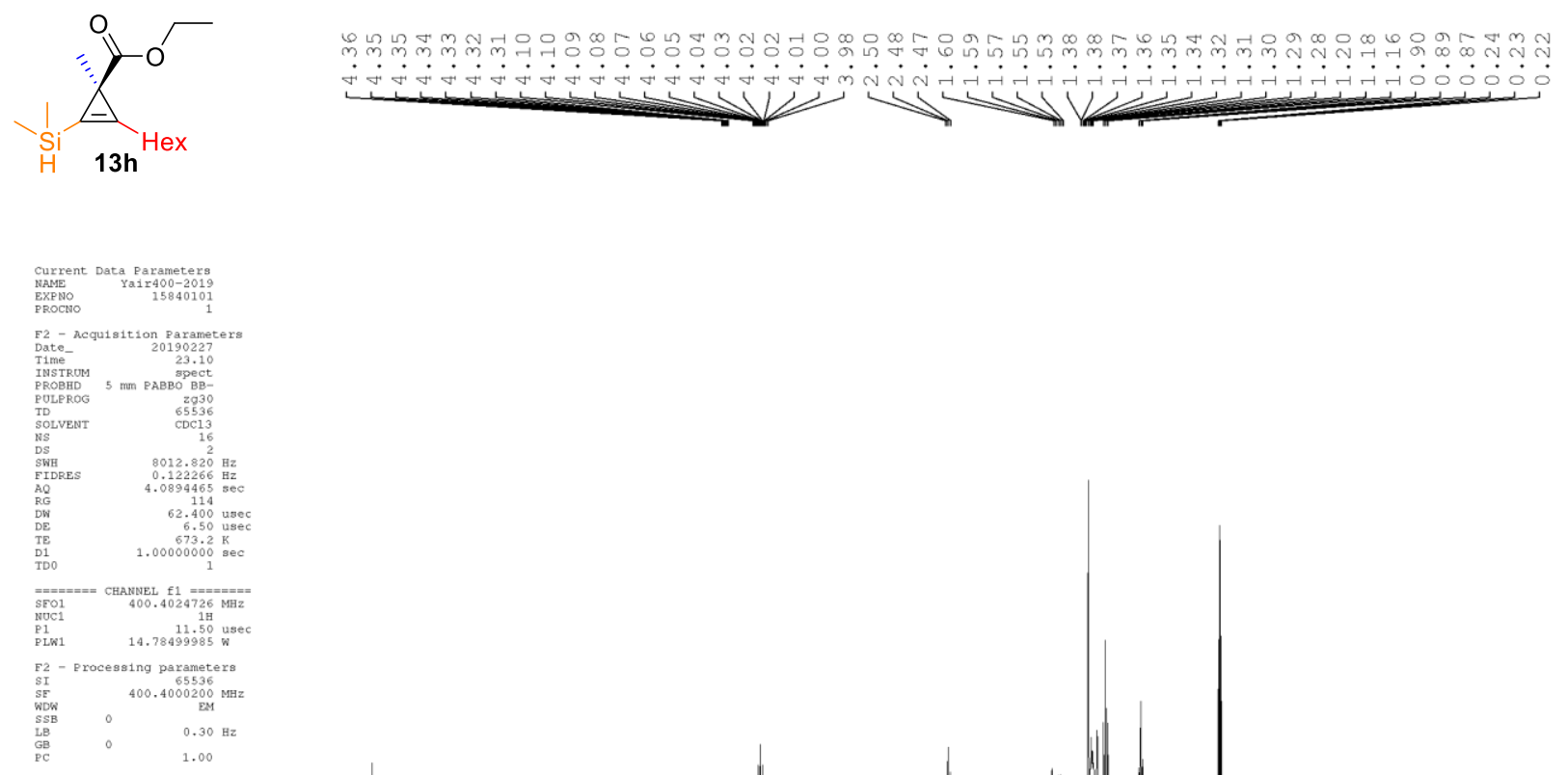

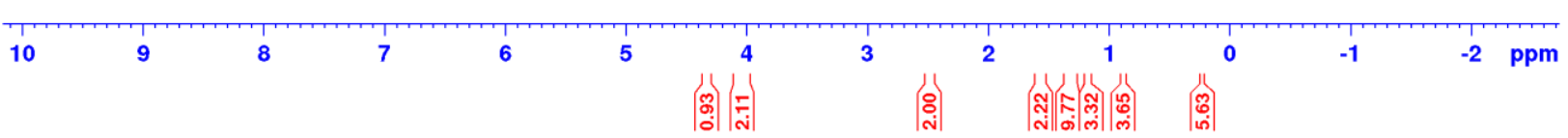
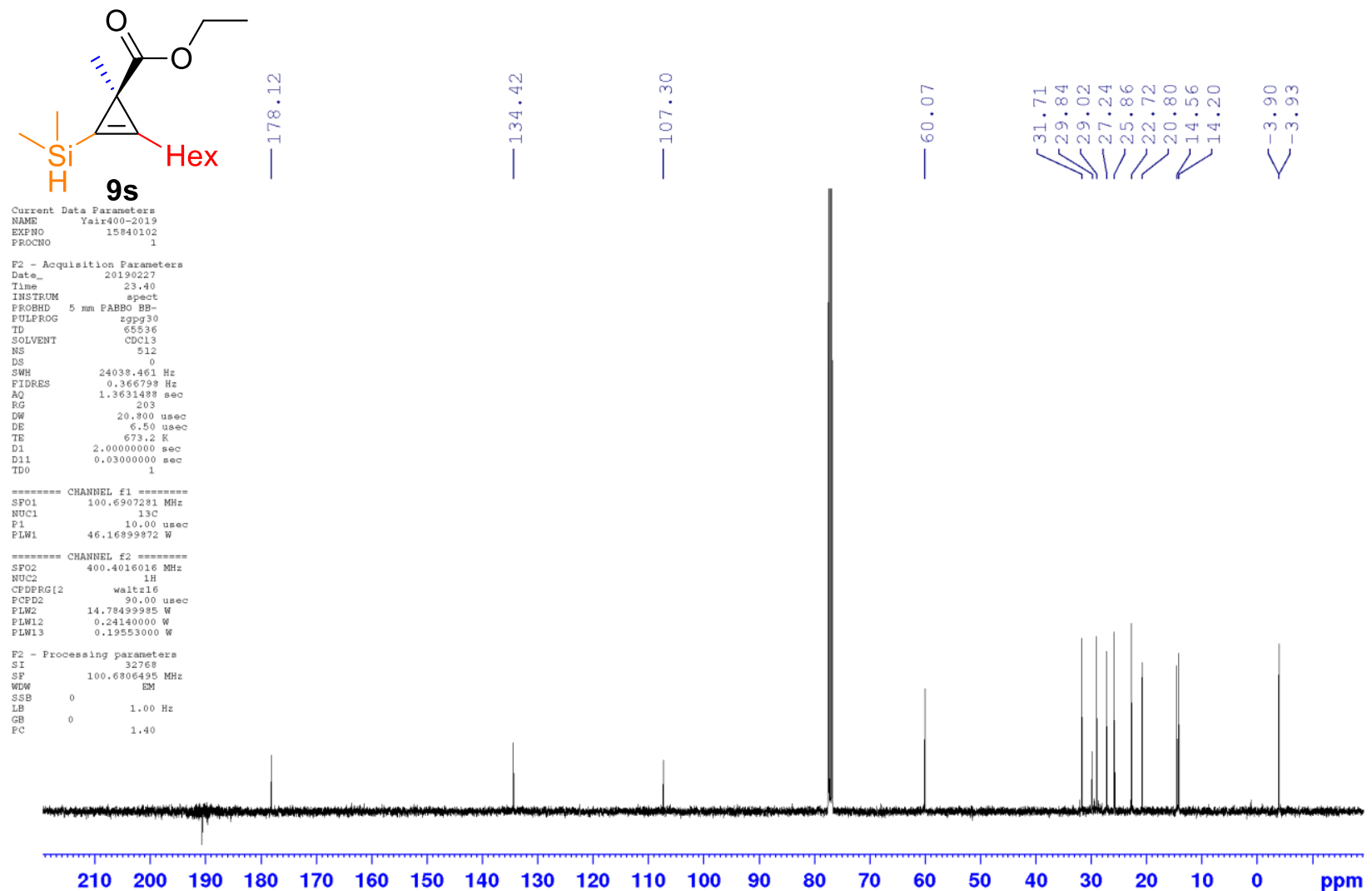